\title{
$S p(4)$ gauge theories on the lattice: Quenched fundamental and antisymmetric fermions
}

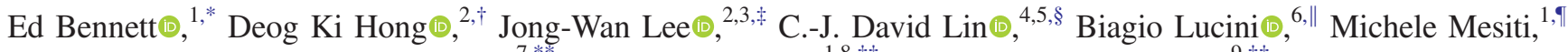 \\ Maurizio Piai®, ${ }^{7, * *}$ Jarno Rantaharju@ ${ }^{1,8,+\dagger}$ and Davide Vadacchino ${ }^{9,+\$}$ \\ ${ }^{1}$ Swansea Academy of Advanced Computing, Swansea University, \\ Bay Campus, SA1 8EN, Swansea, Wales, United Kingdom \\ ${ }^{2}$ Department of Physics, Pusan National University, Busan 46241, Korea \\ ${ }^{3}$ Extreme Physics Institute, Pusan National University, Busan 46241, Korea \\ ${ }^{4}$ Institute of Physics, National Chiao-Tung University, 1001 Ta-Hsueh Road, Hsinchu 30010, Taiwan \\ ${ }^{5}$ Centre for High Energy Physics, Chung-Yuan Christian University, Chung-Li 32023, Taiwan \\ ${ }^{6}$ Department of Mathematics, College of Science, Swansea University, \\ Bay Campus, SA1 8EN, Swansea, Wales, United Kingdom \\ ${ }^{7}$ Department of Physics, College of Science, Swansea University, \\ Singleton Park, SA2 8PP, Swansea, Wales, United Kingdom \\ ${ }^{8}$ Department of Physics and Helsinki Institute of Physics, P.O. Box 64, FI-00014 University of Helsinki, \\ Helsinki, Finland \\ ${ }^{9}$ INFN, Sezione di Pisa, Largo Pontecorvo 3, 56127 Pisa, Italy
}

(Received 17 January 2020; accepted 3 April 2020; published 27 April 2020)

\begin{abstract}
We perform lattice studies of meson mass spectra and decay constants of the $S p(4)$ gauge theory in the quenched approximation. We consider two species of (Dirac) fermions as matter field content, transforming in the 2-index antisymmetric and the fundamental representation of the gauge group, respectively. All matter fields are formulated as Wilson fermions. We extrapolate to the continuum and massless limits and compare to each other the results obtained for the two species of mesons. In the case of two fundamental and three antisymmetric fermions, the long-distance dynamics is relevant for composite Higgs models. This is the first lattice study of this class of theories. The global $S U(4) \times S U(6)$ symmetry is broken to the $S p(4) \times S O(6)$ subgroup, and the condensates align with the explicit mass terms present in the lattice formulation of the theory. The main results of our quenched calculations are that, with fermions in the 2-index antisymmetric representation of the group, the masses squared and decay constant squared of all the mesons we considered are larger than the corresponding quantities for the fundamental representation, by factors that vary between $\sim 1.2$ and $\sim 2.7$. We also present technical results that will be useful for future lattice investigations of dynamical simulations, of composite chimera baryons, and of the approach to large $N$ in the $S p(2 N)$ theories considered. We briefly discuss their high-temperature behavior, where symmetry restoration and enhancement are expected.
\end{abstract}

DOI: 10.1103/PhysRevD.101.074516

\footnotetext{
*e.j.bennett@swansea.ac.uk

†dkhong@pusan.ac.kr

*jwlee823@pusan.ac.kr

\$dlin@mail.nctu.edu.tw

b.lucini@swansea.ac.uk

Tmichele.mesiti@swansea.ac.uk

**m.piai@swansea.ac.uk

†jarno.rantaharju@helsinki.fi

*davide.vadacchino@pi.infn.it
}

Published by the American Physical Society under the terms of the Creative Commons Attribution 4.0 International license. Further distribution of this work must maintain attribution to the author(s) and the published article's title, journal citation, and DOI. Funded by SCOAP.

\section{INTRODUCTION}

In composite Higgs models (CHMs) [1-3], the Higgs fields, responsible for electroweak symmetry breaking, arise as pseudo-Nambu-Goldstone bosons (pNGBs) in a more fundamental theory, hence addressing the little hierarchy problem of generic extensions of the Standard Model (SM) of particle physics. In comparison to the other SM fermions, the top quark has a large mass, making it heavier than the $W$, the $Z$, and even the recently discovered Higgs boson [4,5]. It is then natural to complete the CHM scenario by postulating that also the top quark has composite nature, at least partially, at the fundamental level. The additional model-building dimension added to this framework by (partial) top compositeness yields a 
richness of potential implications that has been explored in the literature on the subject in a range of possible directions and motivates us to study its dynamical origin with nonperturbative techniques. The literature on composite Higgs models is indeed vast (see for instance Refs. [6-40]), especially in connection with dynamical theories characterized by the $S U(4) / S p(4)$ coset (see for instance Refs. [41-61]).

In Ref. [62] (see also Refs. [63,64] and the more recent Refs. [65-68]), some of us proposed a systematic program of exploration of the lattice dynamics of $S p(2 N)$ gauge theories. Our main scientific motivation is the application of the results of such studies to the CHM context. In order to realize also top compositeness, it is necessary to implement on the lattice matter fields with mixed representations. For example, the model discussed in Refs. [12,43] requires that the matter content consists of $N_{f}=2$ Dirac fields transforming in the fundamental representation of $S p(2 N)$, supplemented by $n_{f}=3$ Dirac fields transforming in the antisymmetric representation of $S p(2 N)$. This dynamical system is expected to yield the spontaneous breaking of the $S U(4) \times S U(6)$ global symmetry to its $S p(4) \times S O(6)$ subgroup. The introduction of diagonal mass terms for the fermions is compatible (aligned) with the vacuum structure and provides a degenerate nonvanishing mass for the resulting $5+20$ pNGBs. The lattice treatment of such a system with multiple dynamical fermion representations is a novel arena for lattice gauge theories, and only recently have calculations of this type been published, in the specific context of theories with $S U(4)$ gauge group $[21,27,29,33,38]$.

In this paper, we take a first step in this direction for $S p(2 N)$ gauge theories. We consider the $S p(4)$ gauge theory and treat the two species of fermions in the quenched approximation; only the gluon dynamics is captured by the lattice numerical study, but the operators used to compute the relevant correlation functions involve both types of matter fields. We compute the mass spectra and decay constants of the mesons built both with fundamental and antisymmetric fermions and perform their continuum extrapolation. We compare the properties of mesonic observables obtained with the two representations, which, in the dynamical theory, is important for CHM phenomenology. Since very little is known about the $S p(2 N)$ gauge theories, our quenched study is a first benchmark of these theories and would serve as a starting point for a more extensive and detailed investigation of such models.

We treat the relevant degrees of freedom with a lowenergy effective field theory (EFT) that we employ to analyze the numerical data extrapolated to the continuum limit. The EFT proposed in Ref. [62] for the theory with $S U(4) / S p(4)$ coset is based on the ideas of hidden local symmetry, adapted from Refs. [69-73] (and [74-77]), and supplemented by some simplifying working assumptions.
Here we return to the EFT to improve it and to generalize it to the case of the $S U(6) / S O(6)$ coset.

The paper is organized as follows. In Sec. II, we define the $S p(4)$ theory with field content we are interested in, by writing both the Lagrangian density of the microscopic continuum theory as well as its low-energy EFT description. We devote Sec. III to describing the lattice action we adopt, the Monte Carlo algorithm we employ, and other important aspects of the lattice study we perform, such as scale setting and topology. In Sec. IV we present our results for the calculation of the masses and the (renormalized) decay constants of the lightest mesons in the quenched approximation. We compare the results for quenched fundamental and antisymmetric fermions. We also discuss in Sec. V a first attempt at matching the results to the lowenergy EFT description applicable to pseudoscalar (PS), vector $(\mathrm{V})$ and axial-vector (AV) states. We conclude by summarizing and discussing our main findings and by outlining future avenues for investigation in Sec. VI.

The presentation is complemented by a rather generous set of Appendixes, intended to be of use also beyond the specific aims of this paper, for the research program we are carrying out as a whole. We expose some details and conventions in the treatment of spinors in Appendix A and some technical points about the treatment of massive spin-1 particles in Appendix B. Technical points about the embedding of the SM gauge group in the context of CHMs are highlighted in Appendix C. Appendix D contains some numerical tests of the topological charge history and of its effect on spectral observables, in the illustrative case of a numerical ensemble that has a fine lattice spacing. In Appendix E, besides briefly summarizing some properties of QCD light flavored mesons, we discuss general symmetry properties of the mesons in theories with symmetric cosets, that are important for spectroscopy. We also touch upon possible high-temperature symmetry restoration and enhancement in Appendix E 1. We explicitly write the operators relevant as sources of all the mesons in Appendix F, and in Appendix F 1 we specify the sources of PS, V and AV mesons in the $S U(4) / S p(4)$ case, by adopting a specific choice of $S U(4)$ generators and normalizations.

\section{THE MODEL}

In this section, we describe the specific model of interest, borrowing ideas from Refs. [12,43], and we describe the basic properties of the long-distance EFT description(s) we use later.

\section{A. Continuum microscopic theory}

The $S p(4)$ gauge theory we started to study in Ref. [62] has matter content consisting of two Dirac fermions $Q^{i a}$, where $a=1, \ldots, 4$ is the color index and $i=1,2$ the flavor index, or equivalently four two-component spinors $q^{j a}$ with 
TABLE I. Field content of the microscopic theory $\left(V_{\mu}, q, \psi\right)$ and of the low-energy EFT describing the pNGBs $\left(\Sigma_{6,21}, M_{6,21}\right)$. $S p(4)$ is the gauge group, while $S U(4)$ and $S U(6)$ are the global symmetries. The elementary fields $V_{\mu}$ are gauge bosons, while $q$ and $\psi$ are two-component spinors. $\Sigma_{6}$ and $\Sigma_{21}$ are composite scalar fields. They capture the long-distance dynamics of operators that are bilinear in $q$ and $\psi$, the VEVs of which are responsible for the breaking $S U(4) \rightarrow S p(4)$ and $S U(6) \rightarrow S O(6)$, respectively. The mass matrices $M_{6}$ and $M_{21}$ are treated as scalar spurions, formally transforming as $\sim \overline{6} \sim 6$ of $S U(4)$, and $\sim 2 \overline{1}$ of $S U(6)$, respectively.

\begin{tabular}{lccc}
\hline \hline Fields & $S p(4)$ & $S U(4)$ & $S U(6)$ \\
\hline$V_{\mu}$ & 10 & 1 & 1 \\
$q$ & 4 & 4 & 1 \\
$\psi$ & 5 & 1 & 6 \\
$\Sigma_{6}$ & 1 & 6 & 1 \\
$M_{6}$ & 1 & $6 \sim 6$ & 1 \\
$\Sigma_{21}$ & 1 & 1 & 21 \\
$M_{21}$ & 1 & 1 & 21 \\
\hline \hline
\end{tabular}

$j=1, \ldots, 4$. Following $[12,43]$, we supplement it by three Dirac fermions $\Psi^{i a b}$ transforming in the antisymmetric 2-index representation of $S p(4)$, or equivalently by six two-component spinors $\psi^{j a b}$, with $j=1, \ldots, 6$. The field content is summarized in Table I. The Lagrangian density is

$$
\begin{aligned}
\mathcal{L}= & -\frac{1}{2} \operatorname{Tr} V_{\mu \nu} V^{\mu \nu}+\frac{1}{2}\left(i \overline{Q^{i}}{ }_{a} \gamma^{\mu}\left(D_{\mu} Q^{i}\right)^{a}-i \overline{D_{\mu} Q^{i}}{ }_{a} \gamma^{\mu} Q^{i a}\right) \\
& -M \overline{Q^{i}}{ }_{a} Q^{i a}+\frac{1}{2}\left(i \overline{\Psi^{k}}{ }_{a b} \gamma^{\mu}\left(D_{\mu} \Psi^{k}\right)^{a b}-i \overline{D_{\mu} \Psi^{k}}{ }_{a b} \gamma^{\mu} \Psi^{k a b}\right) \\
& -m \overline{\Psi^{k}{ }_{a b}} \Psi^{k a b} .
\end{aligned}
$$

The covariant derivatives are defined by making use of the transformation properties under the action of an element $U$ of the $S p(4)$ gauge group $-Q \rightarrow U Q$ and $\Psi \rightarrow U \Psi U^{\mathrm{T}}-$ so that

$$
\begin{gathered}
V_{\mu \nu} \equiv \partial_{\mu} V_{\nu}-\partial_{\nu} V_{\mu}+i g\left[V_{\mu}, V_{\nu}\right], \\
D_{\mu} Q^{i}=\partial_{\mu} Q^{i}+i g V_{\mu} Q^{i}, \\
D_{\mu} \Psi^{j}=\partial_{\mu} \Psi^{j}+i g V_{\mu} \Psi^{j}+i g \Psi^{j} V_{\mu}^{\mathrm{T}},
\end{gathered}
$$

where $g$ is the gauge coupling.

The Lagrangian density possesses a global $S U(4)$ symmetry acting on the fundamental fermions $Q$ and a global $S U(6)$ acting on the antisymmetric-representation fermions $\Psi$. The mass terms break them to the $S p(4)$ and $S O(6)$ subgroups, respectively. The unbroken subgroups consist of the transformations that leave invariant the symplectic matrix $\Omega$ and the symmetric matrix $\omega$, respectively, that are defined by

$$
\begin{aligned}
& \Omega=\Omega_{j k}=\Omega^{j k} \equiv\left(\begin{array}{ccccc}
0 & 0 & 1 & 0 \\
0 & 0 & 0 & 1 \\
-1 & 0 & 0 & 0 \\
0 & -1 & 0 & 0
\end{array}\right), \\
& \omega=\omega_{j k}=\omega^{j k} \equiv\left(\begin{array}{cccccc}
0 & 0 & 0 & 1 & 0 & 0 \\
0 & 0 & 0 & 0 & 1 & 0 \\
0 & 0 & 0 & 0 & 0 & 1 \\
1 & 0 & 0 & 0 & 0 & 0 \\
0 & 1 & 0 & 0 & 0 & 0 \\
0 & 0 & 1 & 0 & 0 & 0
\end{array}\right) .
\end{aligned}
$$

By rewriting explicitly the fermion contributions to the Lagrangian density in two-component notation as follows (see Appendix A for the list of conventions about spinors):

$$
\begin{aligned}
Q^{i a} & =\left(\begin{array}{c}
q^{i a} \\
\Omega^{a b}\left(-\tilde{C} q^{i+2 *}\right)_{b}
\end{array}\right), \\
\Psi^{i a b} & =\left(\begin{array}{c}
\psi^{i a b} \\
\Omega^{a c} \Omega^{b d}\left(-\tilde{C} \psi^{i+3 *}\right)_{c d}
\end{array}\right),
\end{aligned}
$$

the global symmetries become manifest:

$$
\begin{aligned}
\mathcal{L}= & -\frac{1}{2} \operatorname{Tr} V_{\mu \nu} V^{\mu \nu}+\frac{1}{2}\left(i\left(q^{j}\right)_{a}^{\dagger} \bar{\sigma}^{\mu}\left(D_{\mu} q^{j}\right)^{a}-i\left(D_{\mu} q^{j}\right)_{a}^{\dagger} \bar{\sigma}^{\mu} q^{j a}\right) \\
& -\frac{1}{2} M \Omega_{j k}\left(q^{j a T} \Omega_{a b} \tilde{C} q^{k b}-\left(q^{j}\right)_{a}^{\dagger} \Omega^{a b} \tilde{C}\left(q^{k *}\right)_{b}\right) \\
& +\frac{1}{2}\left(i\left(\psi^{k}\right)_{a b}^{\dagger} \bar{\sigma}^{\mu}\left(D_{\mu} \psi^{k}\right)^{a b}-i\left(D_{\mu} \psi^{k}\right)_{a b}^{\dagger} \bar{\sigma}^{\mu} \psi^{k a b}\right) \\
& \left.-\frac{1}{2} m \omega_{j k}\left(\psi^{j a b \mathrm{~T}} \Omega_{a c} \Omega_{b d} \tilde{C} \psi^{k c d}-\left(\psi^{j}\right)_{a b}^{\dagger} \Omega^{a c} \Omega^{b d} \tilde{C} \psi^{k *}\right)_{c d}\right) .
\end{aligned}
$$

Of the 15 generators $T^{A}$ of the global $S U(4)$ and 35 generators $t^{B}$ of $S U(6)$, we denote with $A=1, \ldots, 5$ and with $B=1, \ldots, 20$ the broken ones, which obey

$$
\Omega T^{A}-T^{A \mathrm{~T}} \Omega=0, \quad \omega t^{B}-t^{B \mathrm{~T}} \omega=0,
$$

while the unbroken generators with $A=6, \ldots, 15$ and with $B=21, \ldots, 35$ satisfy

$$
\Omega T^{A}+T^{A \mathrm{~T}} \Omega=0, \quad \omega t^{B}+t^{B \mathrm{~T}} \omega=0 .
$$

As described in Appendix C, the Higgs potential in the SM has a global symmetry with group $S U(2)_{L} \times S U(2)_{R} \sim$ $S O(4)$, which in the present case is a subgroup of the unbroken global $S p(4)$. The $S U(3)_{c}$ gauge group characterizing QCD is a subgroup of the unbroken global $S O(6)$. And finally the generator $Y$ of the hypercharge $U(1)_{Y}$ group is a linear combination of one of the generators of 
$S U(2)_{R}$ and of the generator of the additional $U(1)_{X}$ unbroken subgroup of $S O(6)$ that commutes with $S U(3)_{c}$.

\section{B. The pNGB fields}

At low energies, the gauge theory with $S p(4)$ group is best described by an EFT that contains only the fields corresponding to the pNGBs parametrizing the $\frac{S U(4) \times S U(6)}{S p(4) \times S O(6)}$ coset. We define the fields $\Sigma_{6}$ and $\Sigma_{21}$ in terms of the transformation properties of the operators that are responsible for spontaneous symmetry breaking, hence identifying

$$
\begin{gathered}
\Sigma_{6}^{n m} \sim \Omega_{a b} q^{n a \mathrm{~T}} \tilde{C} q^{m b}, \\
\Sigma_{21}^{n m} \sim-\Omega_{a b} \Omega_{c d} \psi^{n a c \mathrm{~T}} \tilde{C} \psi^{m b d} .
\end{gathered}
$$

$\Sigma_{6}$ transforms as the antisymmetric representation of $S U(4)$, and $\Sigma_{21}$ as the symmetric representation of $S U(6)$. We parameterize them in terms of fields $\pi_{6}$ and $\pi_{21}$ as

$$
\begin{gathered}
\Sigma_{6} \equiv e^{2 i \pi_{6} / f_{6}} \Omega=\Omega e^{2 i \pi_{6}^{\mathrm{T}} / f_{6}}, \\
\Sigma_{21} \equiv e^{2 i \pi_{21} / f_{21}} \omega=\omega e^{2 i \pi_{21}^{\mathrm{T}} / f_{21}},
\end{gathered}
$$

where $\pi_{6} \equiv \pi_{6}^{A} T^{A}$ with $A=1, \ldots, 5$ and $\pi_{21} \equiv \pi_{21}^{B} t^{B}$ with $B=1, \ldots, 20$ are Hermitian matrix-valued fields and the generators $T^{A}$ are normalized by the relation $\operatorname{Tr} T^{A} T^{B}=$ $\frac{1}{2} \delta^{A B}=\operatorname{Tr} t^{A} t^{B}$. The decay constants of the pNGBs are denoted by $f_{6}$ and $f_{21}$, and the normalization conventions we adopt correspond to those in which the decay constant in the chiral Lagrangian of QCD is $f_{\pi} \simeq 93 \mathrm{MeV}$.

In order to identify the operators to be included in the Lagrangian density describing the mass-deformed theory, one treats the (diagonal) mass matrices as (nondynamical) spurions $M_{6} \equiv M \Omega$ and $M_{21} \equiv-m \omega$ (see Table I). The vacuum expectation value (VEV) of the operators $\Sigma_{i}$ yields the symmetry-breaking pattern $S U(4) \times S U(6) \rightarrow S p(4) \times$ $S O(6)$, aligned with the explicit breaking terms controlled by $M_{6}$ and $M_{21}$, and hence in the vacuum of the theory we have $\left\langle\pi_{i}\right\rangle=0$.

At the leading order in both the derivative expansion and the expansion in small masses, the Lagrangian densities of the EFT describing the dynamics of the pNGBs of both the $S U(4) / S p(4)$ and $S U(6) / S O(6)$ cosets are given by

$$
\begin{aligned}
\mathcal{L}_{i} & =\frac{f_{i}^{2}}{4} \operatorname{Tr}\left\{\partial_{\mu} \Sigma_{i}\left(\partial^{\mu} \Sigma_{i}\right)^{\dagger}\right\}-\frac{v_{i}^{3}}{4} \operatorname{Tr}\left\{M_{i} \Sigma_{i}\right\}+\text { H.c. } \\
& =\operatorname{Tr}\left\{\partial_{\mu} \pi_{i} \partial^{\mu} \pi_{i}\right\}+\frac{1}{3 f_{i}^{2}} \operatorname{Tr}\left\{\left[\partial_{\mu} \pi_{i}, \pi_{i}\right]\left[\partial^{\mu} \pi_{i}, \pi_{i}\right]\right\}+\cdots
\end{aligned}
$$

$$
+\frac{1}{2} m_{i} v_{i}^{3} \operatorname{Tr}\left(\Sigma_{i} \Sigma_{i}^{\dagger}\right)-\frac{m_{i} v_{i}^{3}}{f_{i}^{2}} \operatorname{Tr} \pi_{i}^{2}+\frac{m_{i} v_{i}^{3}}{3 f_{i}^{4}} \operatorname{Tr} \pi_{i}^{4}+\cdots
$$

for $i=6,21$, and with $m_{6}=M$ and $m_{21}=m .{ }^{1}$ The condensates are parameterized by $v_{6}$ and $v_{21}$, which have dimension of a mass. In the $S U(4) / S p(4)$ case $\operatorname{Tr} \Sigma_{6} \Sigma_{6}^{\dagger}=4$, and in the $S U(6) / S O(6)$ case $\operatorname{Tr} \Sigma_{21} \Sigma_{21}^{\dagger}=6$.

In order to describe the coupling to the Standard Model, one chooses appropriate embeddings for the relevant $S U(2)_{L} \times S U(2)_{R}$ and $S U(3)_{c} \times U(1)_{X}$ groups and promotes the ordinary derivatives to covariant derivatives. By doing so, the irreducible representations of the unbroken $S p(4) \times S O(6)$ can be decomposed in representations of the SM groups (see Appendix C).

Starting from the $S U(4) / S p(4)$ coset, the five pNGBs transform as the fundamental representation of $S O(5) \sim$ $S p(4)$. Because $S O(4) \sim S U(2)_{L} \times S U(2)_{R}$ is a natural subgroup of $S O(5)$, one finds the decomposition $5=1+4$, and hence four of the pNGBs are identified with the SM Higgs doublet, while the one additional degree of freedom is a real singlet of $S U(2)_{L} \times S U(2)_{R}$. In the conventions of [50,62], the latter is denoted by $\pi^{3}$ - or $\pi_{6}^{3}$ if one needs to avoid ambiguity with the set of pNGBs from the $S U(6) / S O(6)$ coset (see also Appendix C 1).

A similar exercise can be performed for the $S U(6) /$ $S O(6)$ coset. By remembering that $S O(6) \sim S U(4)$, the 20 pNGBs transform as the $20^{\prime}$ irreducible representation of this $S U(4)$ [the only self-conjugate among the three representations of $S U(4)$ that has 20 real elements]. ${ }^{2}$ The decomposition of $S U(4)$ in its maximal $S U(3)_{c} \times$ $U(1)_{X}$ subgroup dictates that $20^{\prime}=8+6_{\mathbb{C}}$ (see also Appendix C 1).

\section{EFT: Hidden local symmetry}

This subsection is devoted to the treatment of spin-1 composite states. All irreducible representations coming from the $S U(4) / S p(4)$ theory can be decomposed following the same principles illustrated by the pNGBs, into representations of the groups relevant to SM physics. For example the 10 of $S O(5)$ decomposes as $10=4+6$ of $S O(4)$, so that the composite vector mesons $\mathrm{V}$ of the $S U(4) / S p(4)$ theory (corresponding to the $\rho$ mesons of QCD) decompose into a complex doublet and a complex triplet of $S U(2)_{L} \times S U(2)_{R}$. The axial vectors AV (corresponding to the $a_{1}$ mesons in QCD) transform with the same internal quantum numbers as the pNGBs and hence give rise to a complex doublet and a real singlet. In the

\footnotetext{
${ }^{1}$ In order to make the expansion for the $S U(6) / S O(6)$ formally identical to the $S U(4) / S p(4)$ case, we chose opposite signs in the definition of the mass matrices and condensing operators. The origin for this technical annoyance is the fact that $\Omega^{2}=-\mathbb{1}_{4}$, while $\omega^{2}=\mathbb{1}_{6}$. We also note that one has to exercise caution with the trace of the identity matrix, which may introduce numerical factors that differ in the expansions when traces are taken in products that do not include the group generators.

${ }^{2}$ In the rest of the paper, we will always denote this representation as $20^{\prime}$, for the purpose of avoiding confusion with the representations of the unrelated broken global $S U(4)$.
} 


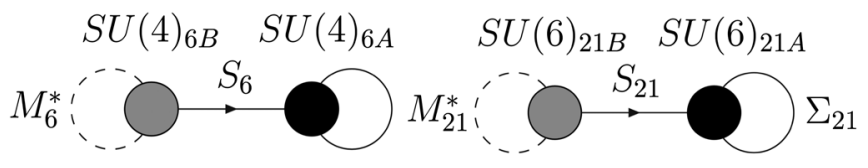

FIG. 1. The moose diagrams representing the low-energy EFT descriptions. On the left $S U(4)_{6 A}$ is gauged, while $S U(4)_{6 B}$ is a global symmetry [including the $S U(2)_{L} \times S U(2)_{R}$ ], and the combination of the nontrivial VEVs of $S_{6}$ and $\Sigma_{6}$ breaks the symmetry to $S p(4)$, giving mass to all the vector mesons and leaving a set of five light pions. On the right, the same principles are applied to $S U(6)_{21 B} \times S U(6)_{21 A}$ and to its breaking to the $S O(6)$ subgroup.

$S U(6) / S O(6)$ coset, the composite vector mesons V transform as the 15 of $S O(6) \sim S U(4)$, which decomposes as $15=1+3_{\mathbb{C}}+8$ of $S U(3)_{c}$, and the axial-vector mesons AV transform as the $20^{\prime}$ of $S O(6)$, which decomposes as $20^{\prime}=8+6_{\mathbb{C}}$ of $S U(3)_{C}$.

We study a reformulation of the low-energy EFT description of the model, that is intended to capture also the behavior of the lightest vector and axial-vector states, in addition to the pNGBs (as in the chiral Lagrangian). It is based on hidden local symmetry [69-73] (see also [74-77]) and illustrated by the diagram in Fig. 1. There are wellknown limitations to the applicability of this type of EFT treatment, which we will discuss in due time.

We consider the two moose diagrams as completely independent from one another. We follow closely the notation of Ref. [62] in describing the $S U(4) / S p(4)$ coset, except for the fact that we include only single-trace operators in the Lagrangian density. Because the breaking is due to the condensate of the operator transforming in the 6 of $S U(4)$, we label all the fields of relevance to the low-energy EFT with a subscript, as in $S_{6}$. The scalar fields $S_{6}$ transform as a bifundamental of $S U(4)_{6 B} \times S U(4)_{6 A}$, while $\Sigma_{6}$ transform as the antisymmetric representations of $S U(4)_{6 A}$. Hence the transformation rules are as follows:

$$
S_{6} \rightarrow U_{6 B} S_{6} U_{6 A}^{\dagger}, \quad \Sigma_{6} \rightarrow U_{6 A} \Sigma_{6} U_{6 A}^{\mathrm{T}},
$$

where $U_{6 A}$ and $U_{6 B}$ are group elements of $S U(4)_{6 A}$ and $S U(4)_{6 B}$, respectively.

The EFT is built by imposing the nonlinear constraints $\Sigma_{6} \Sigma_{6}^{\dagger}=\mathbb{1}_{4}=S_{6} S_{6}^{\dagger}$, which are solved by parameterizing $S_{6}=e^{2 i \sigma_{6} / F}$ and $\Sigma_{6}=e^{2 i \pi_{6} / f} \Omega=\Omega e^{2 i \pi_{6}^{\mathrm{T}} / f} . M_{6}=M \Omega$ is a constant matrix, introducing explicit symmetry breaking. One can think of it as a spurion in the antisymmetric representation of $S U(4)_{6 B}$, so that as a field it would transform according to $M_{6}^{*} \rightarrow U_{6 B} M_{6}^{*} U_{6 B}^{\mathrm{T}}$. The 15 real Nambu-Goldstone fields $\sigma_{6}=\sigma_{6}^{A} T^{A}$ and five real $\pi_{6}=$ $\pi_{6}^{A} T^{A}$ are in part gauged into providing the longitudinal components for the 15 gauge bosons of $S U(4)_{6 A}$, so that only five linear combinations remain in the spectrum as physical pseudoscalars. One then uses $\Sigma_{i}$ and its derivatives, as well as $M_{i}$, to build all possible operators allowed by the symmetries, organizes them as an expansion in derivatives (momenta $p^{2}$ ) and explicit mass terms $(M)$, and writes a Lagrangian density that includes all such operators up to a given order in the expansion. We also restrict attention to operators that can be written as single traces, as anticipated.

Truncated at the next-to-leading order, the Lagrangian density takes the following form, which we borrow from Ref. $[62]^{3}$ :

$$
\begin{aligned}
\mathcal{L}_{6}= & -\frac{1}{2} \operatorname{Tr} A_{\mu \nu} A^{\mu \nu}-\frac{\kappa}{2} \operatorname{Tr}\left\{A_{\mu \nu} \Sigma\left(A^{\mu \nu}\right)^{\mathrm{T}} \Sigma^{*}\right\}+\frac{f^{2}}{4} \operatorname{Tr}\left\{D_{\mu} \Sigma\left(D^{\mu} \Sigma\right)^{\dagger}\right\}+\frac{F^{2}}{4} \operatorname{Tr}\left\{D_{\mu} S\left(D^{\mu} S\right)^{\dagger}\right\} \\
& +b \frac{f^{2}}{4} \operatorname{Tr}\left\{D_{\mu}(S \Sigma)\left(D^{\mu}(S \Sigma)\right)^{\dagger}\right\}+c \frac{f^{2}}{4} \operatorname{Tr}\left\{D_{\mu}\left(S \Sigma S^{\mathrm{T}}\right)\left(D^{\mu}\left(S \Sigma S^{\mathrm{T}}\right)\right)^{\dagger}\right\}-\frac{v^{3}}{8} \operatorname{Tr}\left\{M S \Sigma S^{\mathrm{T}}\right\}+\text { H.c. } \\
& -\frac{v_{1}}{4} \operatorname{Tr}\left\{M\left(D_{\mu} S\right) \Sigma\left(D^{\mu} S\right)^{\mathrm{T}}\right\}-\frac{v_{2}}{4} \operatorname{Tr}\left\{M S\left(D_{\mu} \Sigma\right)\left(D^{\mu} S\right)^{\mathrm{T}}\right\}+\text { H.c. } \\
& -\frac{y_{3}}{8} \operatorname{Tr}\left\{A_{\mu \nu} \Sigma\left[\left(A^{\mu \nu}\right)^{\mathrm{T}} S^{\mathrm{T}} M S-S^{\mathrm{T}} M S A^{\mu \nu}\right]\right\}+\text { H.c. } \\
& -\frac{y_{4}}{8} \operatorname{Tr}\left\{A_{\mu \nu} \Sigma\left[\left(A^{\mu \nu}\right)^{\mathrm{T}} S^{\mathrm{T}} M S+S^{\mathrm{T}} M S A^{\mu \nu}\right]\right\}+\text { H.c. } \\
& +\frac{v_{5}^{2}}{32} \operatorname{Tr}\left\{M S \Sigma S^{\mathrm{T}} M S \Sigma S^{\mathrm{T}}\right\}+\text { H.c. }
\end{aligned}
$$

\footnotetext{
${ }^{3}$ The very last term of the Lagrangian density differs from Ref. [62], as we rewrite the subleading correction to the pion mass in terms of a single-trace operator. The equations giving the masses and decay constants are independent of the dimensionality of the matrices used. We notice also an inconsequential typo in Eq. (2.16) of [62], in which the last term should have a + sign rather than a - sign, in order to be consistent with Eqs. (2.30) and (2.31) of [62] itself.
}

We omitted, for notational simplicity, the subscript " 6 " on all fields and all the parameters. We should stress that we made some simplifications, and omitted some operators, as discussed in [62]. The covariant derivatives introduce the parameter $g_{\mathrm{V}}$, controlling the coupling of the spin-1 states. They can be written as follows: 


$$
D_{\mu} S=\partial_{\mu} S-i S g_{\mathrm{V}} A_{\mu}
$$

and

$$
D_{\mu} \Sigma=\partial_{\mu} \Sigma+i\left[\left(g_{\mathrm{V}} A_{\mu}\right) \Sigma+\Sigma\left(g_{\mathrm{V}} A_{\mu}\right)^{T}\right] .
$$

The analog of Eq. (16) in the $S U(6) / S O(6)$ case is obtained in the same way. The only changes are the replacement of $\Sigma_{6}$ by $\Sigma_{21}$, that now depends on $20 \pi_{21}^{i}$ fields, of $S_{6}$ by $S_{21}$, that depends on $35 \sigma_{21}^{i}$ fields, of $M_{6}$ by $M_{21} \equiv-m \omega$, and of $A_{6 \mu}^{A}$ by the 35 vector bosons $A_{21 \mu}^{A}$ of $S U(6)_{21 A}$. Finally, one must also require the change of sign $\kappa_{21} \leftrightarrow-\kappa_{6}$ in the second term of the Lagrangian, for the same reason explained in footnote 1.

With the conventions outlined above, masses and decay constants are given by the same relations as in Ref. [62], both for the mesons sourced by fundamental and antisymmetric fermion bilinears:

$$
\begin{gathered}
M_{\mathrm{V}}^{2}=\frac{1}{4\left(1+\kappa+m y_{3}\right)} g_{\mathrm{V}}^{2}\left(b f^{2}+F^{2}+2 m v_{1}\right), \\
M_{\mathrm{AV}}^{2}=\frac{1}{4\left(1-\kappa-m y_{4}\right)} g_{\mathrm{V}}^{2}\left(b f^{2}+F^{2}+2 m v_{1}\right) \\
+\frac{g_{V}^{2}}{1-\kappa-m y_{4}}\left(f^{2}+m\left(v_{2}-v_{1}\right)\right), \\
f_{\mathrm{V}}^{2}=\frac{1}{2}\left(b f^{2}+F^{2}+2 m v_{1}\right), \\
f_{\mathrm{AV}}^{2}=\frac{\left(b f^{2}-F^{2}+2 m\left(v_{1}-v_{2}\right)\right)^{2}}{2\left((b+4) f^{2}+F^{2}-2 m v_{1}+4 m v_{2}\right)}, \\
f_{0}^{2}=F^{2}+(b+2 c) f^{2} .
\end{gathered}
$$

The pNGB decay constants obey the following relation ${ }^{4}$ :

$$
f_{\mathrm{PS}}^{2}=f_{0}^{2}-f_{\mathrm{V}}^{2}-f_{\mathrm{AV}}^{2} .
$$

It was observed in Ref. [62] that $f_{0}^{2}=f_{\mathrm{PS}}^{2}+f_{\mathrm{V}}^{2}+f_{\mathrm{AV}}^{2}$ is independent of $m$ as the accidental consequence of the truncations and of the omission of some operators. It was also shown that some of the couplings parameterize the violation of the saturation of the Weinberg sum rules, when truncated at this level-retaining only the lightest excitations sourced by the $\mathrm{V}$ and $\mathrm{AV}$ operators rather than the whole infinite tower of states.

In both the $S U(4) / S p(4)$ as well as $S U(6) / S p(6)$ cosets, truncated at this level the Lagrangian implies that

\footnotetext{
${ }^{4}$ In Ref. [62] we denoted the decay constant of the PS mesons as $f_{\pi}(0)$, to explicitly highlight that this is not the constant that naturally appears in the $\pi \pi \rightarrow \pi \pi$ scattering amplitude.
}

the mass of the pions satisfies a generalized Gell-MannOakes-Renner relation, which reads as follows:

$$
m_{\mathrm{PS}}^{2} f_{\mathrm{PS}}^{2}=m\left(v^{3}+m v_{5}^{2}\right),
$$

which implies a dependence of the condensate on $m$. We notice the presence of the constant $g_{\mathrm{V}}$, which enters the $g_{\mathrm{VPP}}$ coupling between V and two PS states and has an important role in controlling the EFT expansion.

\section{LATTICE MODEL}

The lattice action and its numerical treatment via Monte Carlo methods are the main topics of this section. Most of the material covered here is based upon wellestablished processes, and we discussed its application to our program elsewhere $[62,67]$; hence, we summarize it briefly, mostly for the purpose of defining the notation and language we adopt later in the paper.

\section{A. Lattice definitions}

In the numerical (lattice) studies, we should adopt a discretized four-dimensional Euclidean-space version of Eq. (1). But as we perform our numerical work in the quenched approximation, we only need the pure gauge part of the Lagrangian density, as in pioneering studies of $S p(2 N)$ Yang-Mills theories in Ref. [78]. We employ the standard Wilson action

$$
S_{g} \equiv \beta \sum_{x} \sum_{\mu<\nu}\left(1-\frac{1}{4} \operatorname{Re} \operatorname{Tr} \mathcal{P}_{\mu \nu}(x)\right),
$$

where $\beta=8 / g^{2}$ is the bare lattice coupling and the trace is over color indices. The elementary plaquette $\mathcal{P}_{\mu \nu}$ is a pathordered product of (fundamental) link variables $U_{\mu}(x)$, the group elements of $S p(4)$, and reads as follows:

$$
\mathcal{P}_{\mu \nu}(x) \equiv U_{\mu}(x) U_{\nu}(x+\hat{\mu}) U_{\mu}^{\dagger}(x+\hat{\nu}) U_{\nu}^{\dagger}(x) .
$$

Given the action in Eq. (26), we generate the gauge configurations by implementing a heat bath (HB) algorithm with microcanonical overrelaxation updates. Technical details, including the modified Cabbibo-Marinari procedure [79] and the resymplectization process we adopted, can be found in Refs. [62,65]. The HIRep code [80], appropriately adapted to the requirements of this project, is used for the numerical calculations.

The pure $S p(4)$ Yang-Mills lattice theory at any values of $\beta$ can in principle be connected smoothly to the continuum, as no evidence of bulk transitions has been found [78]. In this study, we work in the regime with $\beta>7.5$. In a previous publication [62], some of us used two values of the coupling $(\beta=7.62$ and $\beta=8.0)$ and performed preliminary studies of the meson spectrum with fermions in the fundamental representation, in the 
TABLE II. List of ensembles used for quenched calculations. For each ensemble, we report the bare coupling $\beta$, the lattice size $N_{t} \times N_{s}^{3}$, the average plaquette $\langle P\rangle$ and the gradient-flow scale $w_{0} / a=1 / \hat{a}$.

\begin{tabular}{lllll}
\hline \hline Ensemble & \multicolumn{1}{c}{$\beta$} & $N_{t} \times N_{s}^{3}$ & \multicolumn{1}{c}{$\langle P\rangle$} & \multicolumn{1}{c}{$w_{0} / a$} \\
\hline QB1 & 7.62 & $48 \times 24^{3}$ & 0.60192 & $1.448(3)$ \\
QB2 & 7.7 & $60 \times 48^{3}$ & 0.608795 & $1.6070(19)$ \\
QB3 & 7.85 & $60 \times 48^{3}$ & 0.620381 & $1.944(3)$ \\
QB4 & 8.0 & $60 \times 48^{3}$ & 0.630740 & $2.3149(12)$ \\
QB5 & 8.2 & $60 \times 48^{3}$ & 0.643228 & $2.8812(21)$ \\
\hline \hline
\end{tabular}

quenched limit. In order to carry out the continuum extrapolation, here we extend those studies by including three additional values of the bare lattice coupling, $\beta=7.7$, $7.85,8.2$. The four-dimensional Euclidean lattice has size $N_{t} \times N_{s}^{3}$, with $N_{t}$ and $N_{s}$ the temporal and spatial extents, respectively. We impose periodic boundary conditions in all directions for the gauge fields. While for the ensemble at $\beta=7.62$ we reuse the configurations generated on a $48 \times 24^{3}$ lattice already employed in the quenched calculations in Ref. [62], for all the other values of the coupling we generate new configurations with $60 \times 48^{3}$ lattice points. For each lattice coupling we generate 200 gauge configurations, separated by 12 trajectories ${ }^{5}$ between adjacent configurations. To ensure thermalization, we discard the first 600 trajectories. In Table II we summarize the ensembles. In addition to the ensemble name, the lattice coupling and the lattice size, we also present two measured quantities: the average plaquette $\langle P\rangle$ and the gradient-flow scale $w_{0} / a$ in lattice units. The former is defined by $\langle P\rangle \equiv \operatorname{Re} \sum_{x} \sum_{\mu<\nu} \operatorname{Tr} \mathcal{P}_{\mu \nu}(x) /\left(24 \times N_{t} \times N_{s}^{3}\right)$, while the latter will be defined and discussed in the next subsection. The statistical uncertainties are estimated by using a standard bootstrapping technique for resampling, which will also be applied to the rest of this work.

\section{B. Scale setting and topology}

In numerical lattice calculations, all dimensional quantities can be written in terms of the lattice spacing $a$, for example by defining a dimensionless mass as $m^{\text {latt }}=m a$. But in taking the continuum limit, the lattice spacing vanishes, $a \rightarrow 0$. Hence, in order to connect the lattice observables to continuum ones, we have to set a common physical scale that allows the comparison. We adopt as our scale-setting method Lüscher's gradient-flow (GF) scheme, using the definition of Wilson flow in Ref. [81] (see also

\footnotetext{
${ }^{5}$ Conventionally, for heat bath simulations like those used in this work, a full update of the lattice link variables is called a sweep rather than a trajectory. However, to match the terminology of our dynamical simulations $[62-64,67,68]$, we use the term trajectory for a full lattice gauge field update also in the present context.
}

Refs. [82-84]). This method is particularly suitable for the purpose of this work, since it relies on theoretically defined quantities that do not require direct experimental input.

The scale-setting procedure with the GF scheme in $S p(4)$ theories has been first discussed in Ref. [62], both for the pure Yang-Mills and for the theory with two fundamental Dirac fermions (see also $[63,67]$ ). We follow the same procedure throughout this work: we define the flow scale $w_{0}$ by $\left.\mathcal{W}\right|_{t=w_{0}^{2}}=\mathcal{W}_{0}$ [85], where $\mathcal{W}(t)$ is the derivative of the action density built from gauge fields at nonzero fictitious flow time $t$. The reference value $\mathcal{W}_{0}=$ 0.35 has been chosen to minimize both discretization and finite-volume effects [62] (though with the caveats discussed in Refs. [86-88]). We also choose a four-plaquette clover for the definition of the field-strength tensors [81]. The resulting values of the flow scale in lattice units $w_{0} / a$ are shown in Table II.

We measure the history of the topological charge $Q$ to monitor the possible emergence of topological freezing, which might affect spectral measurements $[89,90]$ (see also Refs. [91,92]). Since $Q \equiv \sum_{x} \frac{1}{32 \pi^{2}} \epsilon_{\mu \nu \rho \sigma} \operatorname{Tr}\left\{U_{\mu \nu}(x) U_{\rho \sigma}(x)\right\}$ is dominated by ultraviolet (UV) fluctuations when calculated directly on the configurations in ensembles QB1-5, configurations that have been smoothed by the gradient flow are instead used. $Q$ is measured at the point in the flow such that the smoothing radius $\sqrt{8 t}=L / 2$.

In Fig. 2 we present the histories and histograms of $Q$ along the Markov chain for all ensembles in Table II, the latter of which is fitted with the Gaussian fit form $n(Q) \propto \exp \left(-\frac{\left(Q-Q_{0}\right)^{2}}{2 \sigma^{2}}\right)$. In Table III we present the results of this fit and the exponential autocorrelation time $\tau_{\text {exp }}$ calculated via a fit to the autocorrelation function of $Q$. In the five ensembles, there is no clear evidence of a freezeout of the topology; the histograms clearly show sampling from multiple topological sectors, and the distributions are peaked within $1 \sigma$ of $Q=0$.

However, as we move to finer lattice spacing, we observe that the autocorrelation time of the topological charge grows significantly; in the case of QB5, this has grown to around 34 configurations. In this case specifically $Q_{0}=$ -4.12 is also marginal compared to $\sigma=4.81$. This effect may be due to the fact that a change of the discrete global quantity $Q$ by local updates becomes disfavored in the approach to the continuum limit.

To verify that this increasing $\tau_{\text {exp }}$ and marginal $Q_{0}$ do not affect the spectroscopic results we obtain from these ensembles, we generate an additional ensemble QB $\overline{5}$ of 2400 trajectories starting from the last configuration in QB5. We repeated the measurements of meson masses and decay constants, and of the topological charge history, on 200 configurations sampled from QB $\overline{5}$. While the value of $Q_{0}$ differs between the two ensembles, the meson masses and decay constants do not show significant deviations (beyond the statistical fluctuations). We report these tests in 

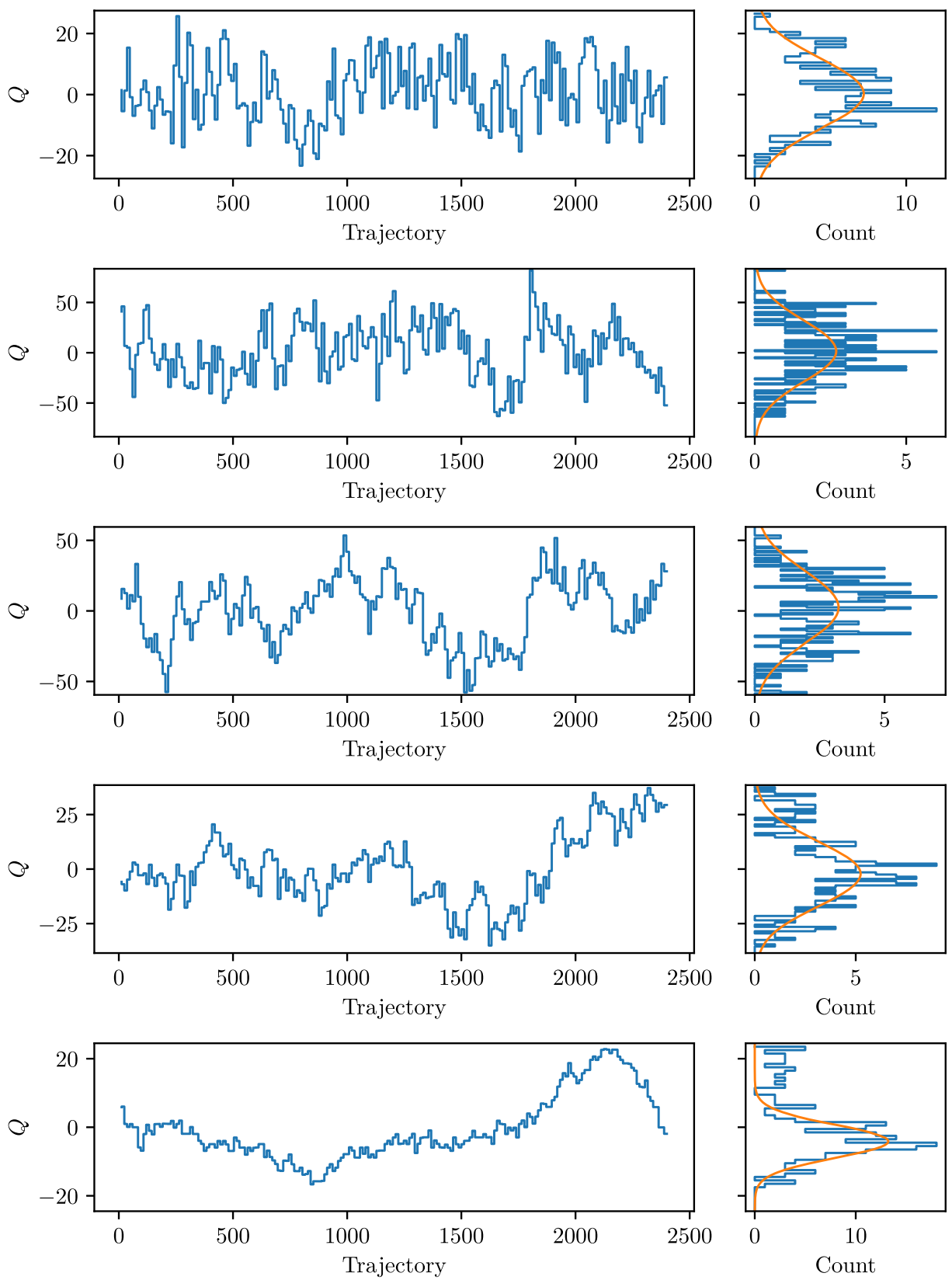

FIG. 2. Topological charge histories (left panels), and histograms (right panels), for the five ensembles QB1-QB5, listed from top to bottom. Fitted parameters are given in Table III. In the plots we show $Q$ evaluated only on the configurations used in the data analysis.

TABLE III. Fitted parameters from topological charge histories; see also Fig. 2. The autocorrelation time $\tau_{\exp }$ is expressed in units of consecutive configurations.

\begin{tabular}{lccc}
\hline \hline & $Q_{0}$ & $\sigma$ & $\tau_{\exp }$ \\
\hline QB1 & $0.29(92)$ & $11.43(94)$ & $1.35(21)$ \\
QB2 & $1.6(2.3)$ & $30.2(2.3)$ & $2.95(24)$ \\
QB3 & $2.5(2.3)$ & $25.4(2.3)$ & $7.73(12)$ \\
QB4 & $-2.2(1.1)$ & $14.7(1.1)$ & $15.79(65)$ \\
QB5 & $-4.12(46)$ & $4.81(46)$ & $34.1(1.7)$ \\
\hline \hline
\end{tabular}

detail in Appendix D. This suggests that any systematic effect associated with the long autocorrelation time of the topological charge and the marginal $Q_{0}$ on the spectroscopy is comfortably smaller than the statistical error for the ensembles and observables we study, and we use ensembles QB1-5 for the remainder of the analysis.

\section{OF QUENCHED MESONS}

In this section, we present the main numerical results of our study. We start by defining the mesonic two-point correlation functions that are computed numerically, and 
TABLE IV. Interpolating operators $\mathcal{O}_{M}$ built of Dirac fermions on the fundamental $Q^{i a}$ and antisymmetric $\Psi^{k a b}$. We show explicitly the flavor indices $i, j=1,2$ and $k, m=1,2,3$, while color and spinor indices are implicit and summed over. We also show the $J^{P}$ quantum numbers, the corresponding QCD mesons sourced by the analogous operator, and the irreducible representation of the unbroken global $S p(4) \times S O(6)$ spanned by the meson (see also [44]). We indicate in parentheses other nontrivial representations that are obtained with the same operator structure but that we do not study in this paper as they source heavier states. The singlets [1 of both $S p(4)$ and $S O(6)]$ are ignored, as we choose to analyze only the operators with $i \neq j$ or $k \neq m$. More details about the symmetries can be found in Appendix E, and the details of a specific choice of basis for the global $S U(4)$ are presented in Appendix F.

\begin{tabular}{|c|c|c|c|c|c|}
\hline Label $M$ & Interpolating operator $\mathcal{O}_{M}$ & Meson in QCD & $J^{P}$ & $S p(4)$ & $S O(6)$ \\
\hline PS & $\overline{Q^{i}} \gamma_{5} Q^{j}$ & $\pi$ & $0^{-}$ & $5(+1)$ & 1 \\
\hline S & $\overline{Q^{i}} Q^{j}$ & $a_{0}$ & $0^{+}$ & $5(+1)$ & 1 \\
\hline $\mathrm{V}$ & $\overline{Q^{i}} \gamma_{\mu} Q^{j}$ & $\rho$ & $1^{-}$ & 10 & 1 \\
\hline $\mathrm{T}$ & $\overline{Q^{i}} \gamma_{0} \gamma_{\mu} Q^{j}$ & $\rho$ & $1^{-}$ & $10(+5+1)$ & 1 \\
\hline AV & $\overline{Q^{i}} \gamma_{5} \gamma_{\mu} Q^{j}$ & $a_{1}$ & $1^{+}$ & $5(+1)$ & 1 \\
\hline AT & $\overline{Q^{i}} \gamma_{5} \gamma_{0} \gamma_{\mu} Q^{j}$ & $b_{1}$ & $1^{+}$ & $10(+5+1)$ & 1 \\
\hline ps & $\overline{\Psi^{k}} \gamma_{5} \Psi^{m}$ & $\pi$ & $0^{-}$ & 1 & $20^{\prime}(+1)$ \\
\hline s & $\overline{\Psi^{k}} \Psi^{m}$ & $a_{0}$ & $0^{+}$ & 1 & $20^{\prime}(+1)$ \\
\hline $\mathrm{v}$ & $\bar{\Psi}^{k} \gamma_{\mu} \Psi^{m}$ & $\rho$ & $1^{-}$ & 1 & 15 \\
\hline $\mathrm{t}$ & $\overline{\Psi^{k}} \gamma_{0} \gamma_{\mu} \Psi^{m}$ & $\rho$ & $1^{-}$ & 1 & $15\left(+20^{\prime}+1\right)$ \\
\hline av & $\overline{\Psi^{k}} \gamma_{5} \gamma_{\mu} \Psi^{m}$ & $a_{1}$ & $1^{+}$ & 1 & $20^{\prime}(+1)$ \\
\hline at & $\bar{\Psi}^{k} \gamma_{5} \gamma_{0} \gamma_{\mu} \Psi^{m}$ & $b_{1}$ & $1^{+}$ & 1 & $15\left(+20^{\prime}+1\right)$ \\
\hline
\end{tabular}

the observables we extract from them, namely the meson masses and decay constants. We provide some technical details about the otherwise standard procedure we follow, in order to clarify how different representations of the gauge group are implemented. Perturbative renormalization of the decay constants is summarized toward the end of Sec. IV A. We perform continuum extrapolations with the use of Wilson chiral perturbation theory $(\mathrm{W} \chi \mathrm{PT})$ in Sec. IV B. We devote Secs. IV C and IV D to present the numerical results for the mesons made of fermions transforming in the fundamental and 2-index antisymmetric representations, respectively, and conclude with a comparison of the two representations in Sec. IV E. For practical reasons, in this section we specify our results to the theory with $N_{f}=2$ fermions on the fundamental representation and $n_{f}=3$ on the antisymmetric, though the results of the quenched calculations apply for generic $N_{f}$ and $n_{f}$.

\section{A. Correlation functions}

We extract masses and decay constants of the lightest flavored spin- 0 and spin- 1 mesons from the corresponding Euclidean two-point correlation functions of operators $\mathcal{O}_{M}$ involving Dirac fermions $Q$ transforming in the fundamental and $\Psi$ in the 2-index antisymmetric representation, as listed in Table IV. In the table, color and spin indices are implicitly summed over, while the flavor indices $i \neq j$ $(k \neq m)$ are chosen. The operators of the form $\overline{Q^{i}} \Gamma_{M} Q^{j}$ are gauge invariant and they source the meson states $M$. Spin and parity $J^{P}$ are determined by the choice of $\Gamma_{M}$. The operators built with $\Gamma=\gamma_{5}, \gamma_{\mu}, \gamma_{5} \gamma_{\mu}$, with $\mu=1,2,3$, correspond to the pseudoscalar (PS), vector (V), and axialvectors (AV) mesons, respectively. They appeared in the EFT discussion in Sec. II C. For all of them, we measure both the masses and the decay constants of the particles that they source. For completeness, we also calculate the correlation functions built with $\Gamma=\mathbb{1}_{4}, \gamma_{0} \gamma_{\mu}, \gamma_{5} \gamma_{0} \gamma_{\mu}$, which refer to scalar (S), (antisymmetric) tensor (T), and axial tensor (AT), but we extract only the masses of the lightest states sourced by these operators. The operators $\bar{\Psi}^{k} \Gamma_{M} \Psi^{m}$ are defined and classified in the same way, except that we denote them with lowercase letters as ps, v, av, s, t, and at, respectively. In Table IV, we also show the irreducible representation of the unbroken global symmetry $S p(4) \times S O(6)$, as well as the corresponding mesons in $\mathrm{QCD}$, to provide intuitive guidance to the reader. We also recall that because of the (pseudo)real nature of the representations we use, there is no difference between meson and diquark operators. More details about the classification of the mesons and the relation between four-component and two-component spinors can be found in Appendixes $\mathrm{E}$ and $\mathrm{F}$.

The two-point correlation functions at positive Euclidean time $t$ and vanishing momentum $\vec{p}$ can be written as

$$
C_{M, M^{\prime}}(t) \equiv \sum_{\vec{x}}\left\langle 0\left|\mathcal{O}_{M}(\vec{x}, t) \mathcal{O}_{M^{\prime}}^{\dagger}(\overrightarrow{0}, 0)\right| 0\right\rangle
$$


We extract physical observables from these objects. In most of our calculations we set $M=M^{\prime}$, with the exception of the extraction of the pseudoscalar decay constant, which involves both $\mathcal{O}_{\mathrm{PS}}$ and $\mathcal{O}_{\mathrm{AV}}\left(\mathcal{O}_{\mathrm{ps}}\right.$ and $\mathcal{O}_{\text {av }}$ in the case of fermions $\Psi$ ). The standard procedure requires rewriting $C(t)$ in terms of fermion propagators $S_{b \beta}^{a \alpha}(x) \equiv$ $\left\langle Q^{a \alpha}(x) \bar{Q}_{b \beta}(0)\right\rangle$ (and analogous expressions for the propagators involving $\Psi$ ), to yield

$$
C_{M, M^{\prime}}(t)=-\sum_{\vec{x}} \operatorname{Tr}\left[\Gamma_{M} S(x) \Gamma_{M^{\prime}} \gamma_{5} S^{\dagger}(x) \gamma_{5}\right],
$$

where the trace is over both spinor indices $\alpha, \beta$ and gauge indices $a, b$.

In the simplest case of a point source, the fermion propagator $D^{R}$ ( $R$ labeling the fermion representation) is calculated by solving the Dirac equation

$$
D_{a \alpha, b \beta}^{R}(x, y) S_{c \gamma}^{b \beta}(y)=\delta_{x, 0} \delta_{\alpha \gamma} \delta_{a c} .
$$

In order to improve the signal, in our numerical studies throughout this work we use the $Z_{2} \times Z_{2}$ single time slice stochastic wall sources [93] with three different sources considered individually for each configuration, instead of the point sources, on the right-hand side of Eq. (30).

In all the spectroscopic measurements using quenched ensembles, we use the (unimproved) Wilson action for the fermions. The corresponding massive Wilson-Dirac operator in the fundamental representation $D^{F}$ is defined by its action on the fermions $Q$, that takes the form

$$
\begin{aligned}
D^{F} Q(x) \equiv & \left(4 / a+m_{0}\right) Q(x)-\frac{1}{2 a} \sum_{\mu}\left\{\left(1-\gamma_{\mu}\right) U_{\mu}(x) Q(x+\hat{\mu})\right. \\
& \left.+\left(1+\gamma_{\mu}\right) U_{\mu}(x-\hat{\mu}) Q(x-\hat{\mu})\right\},
\end{aligned}
$$

where $U_{\mu}(x)$ are the link variables in the fundamental representation of $S p(4), a$ is the lattice spacing, and $\hat{\mu}$ is the unit vector in the spacelike direction $\mu$.

In order to construct the Dirac operator $D^{A S}$ for fermion fields $\Psi^{a b}$ in the 2-index antisymmetric representation, we follow the prescription in [80]. For $S p(2 N)$, we define an orthonormal basis $e_{A S}^{(a b)}$ [with the multi-index $(a b)$ running over ordered pairs with $1 \leq a<b \leq 2 N$ ] for the appropriate vector space of $2 N \times 2 N$ antisymmetric matrices. The $N(2 N-1)-1$ such matrices have the following nonvanishing entries. For $b=N+a$ and $2 \leq a \leq N$

$$
\left(e_{A S}^{(a b)}\right)_{c, N+c} \equiv-\left(e_{A S}^{(a b)}\right)_{N+c, c} \equiv\left\{\begin{array}{l}
\frac{1}{\sqrt{2 a(a-1)}}, \text { for } c<a, \\
\frac{-(a-1)}{\sqrt{2 a(a-1)}}, \text { for } c=a,
\end{array}\right.
$$

and for $b \neq N+a$

$$
\left(e_{A S}^{(a b)}\right)_{c d} \equiv \frac{1}{\sqrt{2}}\left(\delta_{a c} \delta_{b d}-\delta_{b c} \delta_{a d}\right) .
$$

The main difference compared to the case of $S U(N)$ is that the base $e_{A S}$ is $\Omega$-traceless, satisfying $\Omega^{d c}\left(e_{A S}^{(a b)}\right)_{c d}=0$. In the $S p(4)$ case, one can verify that the resulting five nonvanishing matrices satisfy the orthonormalization condition $\operatorname{Tr} e_{A S}^{(a b)} e_{A S}^{(c d)}=-\delta^{(a b)(c d)}$, while the matrix $e_{A S}^{(13)}$ vanishes identically. The explicit form of the antisymmetric link variables $U_{\mu}^{A S}(x)$ descends from the fundamental link variables $U_{\mu}(x)$, as

$$
\begin{gathered}
\left(U_{\mu}^{A S}\right)_{(a b)(c d)}(x) \equiv \operatorname{Tr}\left[\left(e_{A S}^{(a b)}\right)^{\dagger} U_{\mu}(x) e_{A S}^{(c d)} U_{\mu}^{\mathrm{T}}(x)\right], \\
\text { with } \quad a<b, c<d .
\end{gathered}
$$

Finally, the Dirac operator for the 2-index antisymmetric representation $D^{A S}$ is obtained by replacing $\left(U_{\mu}\right)_{a b}$ by $\left(U_{\mu}^{A S}\right)_{(a b)(c d)}$ and $Q$ by $\Psi$ in Eq. (31).

Masses and decay constants for the mesons are extracted from the asymptotic behavior of $C_{M, M^{\prime}}(t)$ at large Euclidean time. We assume it to be dominated by a single mesonic state. If $M=M^{\prime}$, for all meson interpolating operators we can write

$$
C_{M, M}(t) \stackrel{t \rightarrow \infty}{\longrightarrow} \frac{\left|\left\langle 0\left|\mathcal{O}_{M}\right| M\right\rangle\right|^{2}}{2 m_{M}}\left[e^{-m_{M} t}+e^{-m_{M}(T-t)}\right],
$$

where $T$ is the temporal extent of the lattice. In our conventions, the meson states $|M\rangle$ are normalized by writing $M=M^{A} T^{A}$, with $T^{A}$ the generators of the global $S U\left(2 N_{f}\right)$ or $S U\left(2 n_{f}\right)$ symmetry. The value of the pseudoscalar decay constant in QCD in these conventions would be $f_{\mathrm{PS}}=f_{\pi} \simeq 93 \mathrm{MeV}$. We also consider the correlator defined with $M=\mathrm{PS}$ and $M^{\prime}=\mathrm{AV}$, for which the large-time behavior is given by

$C_{\mathrm{PS}, \mathrm{AV}}(t) \stackrel{t \rightarrow \infty}{\longrightarrow} \frac{\left\langle 0\left|\mathcal{O}_{\mathrm{AV}}\right| \mathrm{PS}\right\rangle\left\langle 0\left|\mathcal{O}_{\mathrm{PS}}\right| \mathrm{PS}\right\rangle^{*}}{2 m_{\mathrm{PS}}}\left[e^{-m_{\mathrm{PS}} t}-e^{-m_{\mathrm{PS}}(T-t)}\right]$,

having restricted attention to the components of the $\mathrm{AV}$ operator with index $\mu=1,2,3$.

We parameterize the vacuum-to-meson matrix elements for fundamental fermions in such a way that the decay constants $f_{M}$ obey the following relations:

$$
\begin{aligned}
\left\langle 0\left|\mathcal{O}_{\mathrm{AV}}\right| \mathrm{PS}\right\rangle & =\left\langle 0\left|\bar{Q}_{1} \gamma_{5} \gamma_{\mu} Q_{2}\right| \mathrm{PS}\right\rangle \equiv f_{\mathrm{PS}} p_{\mu}, \\
\left\langle 0\left|\mathcal{O}_{\mathrm{V}}\right| \mathrm{V}\right\rangle & =\left\langle 0\left|\bar{Q}_{1} \gamma_{\mu} Q_{2}\right| \mathrm{V}\right\rangle \equiv f_{\mathrm{V}} m_{\mathrm{V}} \epsilon_{\mu}, \\
\left\langle 0\left|\mathcal{O}_{\mathrm{AV}}\right| \mathrm{AV}\right\rangle & =\left\langle 0\left|\bar{Q}_{1} \gamma_{5} \gamma_{\mu} Q_{2}\right| \mathrm{AV}\right\rangle \equiv f_{\mathrm{AV}} m_{\mathrm{AV}} \epsilon_{\mu},
\end{aligned}
$$


where the polarization vector $\epsilon_{\mu}$ is transverse to the momentum $p_{\mu}$ and normalized by $\epsilon_{\mu}^{*} \epsilon^{\mu}=1$. (For operators constituted by antisymmetric fermions we replace the fields $Q$ by $\Psi$.) For spin-1 $\mathrm{V}$ and $\mathrm{AV}$ mesons we extract both masses and decay constants from Eqs. (35) and (37). In the case of the pseudoscalar meson, we determine the masses and decay constants by combining Eqs. (35) with $M=\mathrm{PS}$, Eq. (36) and Eq. (37).

The matrix elements at finite lattice spacing $a$ have to be renormalized. For Wilson fermions, the axial and vector currents receive multiplicative (finite) renormalization. The renormalization factors $Z_{\mathrm{A}}$ and $Z_{\mathrm{V}}$ are defined by the relations

$f_{\mathrm{PS}}=Z_{\mathrm{A}} f_{\mathrm{PS}}^{\text {bare }}, \quad f_{\mathrm{V}}=Z_{\mathrm{V}} f_{\mathrm{V}}^{\text {bare }}, \quad f_{\mathrm{AV}}=Z_{\mathrm{A}} f_{\mathrm{AV}}^{\text {bare }}$.

In this work we determine the renormalization factors via one-loop perturbative matching, and for Wilson fermions the relevant matching coefficients are written as [94]

$$
Z_{\mathrm{A}(\mathrm{V})}=1+C(R)\left(\Delta_{\Sigma_{1}}+\Delta_{\Gamma}\right) \frac{\tilde{g}^{2}}{16 \pi^{2}},
$$

where $\Gamma=\gamma_{5} \gamma_{\mu}$ for $Z_{\mathrm{A}}$ and $\Gamma=\gamma_{\mu}$ for $Z_{\mathrm{V}}$. The eigenvalues of the quadratic Casimir operators with fermions in the fundamental and antisymmetric representations of $S p(4)$ are $C(F)=5 / 4$ and $C(A S)=2$, respectively. The matching factors in Eq. (39) are computed by one-loop integrals within the continuum $\overline{\mathrm{MS}}$ (modified minimal subtraction) regularization scheme. The resulting numerical values are $\Delta_{\Sigma_{1}}=-12.82, \Delta_{\gamma_{\mu}}=-7.75$ and $\Delta_{\gamma_{5} \gamma_{\mu}}=-3.0$ [62,94]. Following the prescription in Ref. [95], in order to improve the convergence of perturbative expansion we replace the bare coupling $g$ by the tadpole improved coupling defined as $\tilde{g}^{2}=g^{2} /\langle P\rangle .\langle P\rangle$ is the average plaquette value, and this procedure removes large tadpole-induced additive renormalization arising with Wilson fermions.

\section{B. Continuum extrapolation}

Extrapolations to the continuum limit are carried out following the same procedure as in Ref. [67]. We borrow the ideas of tree-level Wilson chiral perturbation theory $(\mathrm{W} \chi \mathrm{PT})$, which we truncate at the next-to-leading order (NLO) in the double expansion in fermion mass and lattice spacing [96,97] (see also Ref. [98], as well as [99,100], though written in the context of improvement). Tree-level results for the full theory can be extended to (partially) quenched calculations, since quenching effects only arise from integrals in fermion loops [97]. But we cannot a priori determine the range of validity of tree-level $\mathrm{W} \chi \mathrm{PT}$ at NLO. On the one hand, if we were too close to the chiral limit, we would need to include loop integrals (the well-known chiral $\operatorname{logs}$ ). On the other hand, if we were in the heavy mass regime, then we would need to include more higher-order terms. As we will discuss later, most of our data sit somewhere in between these two extrema, and as a consequence we can empirically find appropriate ranges of fermion mass over which tree-level NLO $\mathrm{W}_{\chi} \mathrm{PT}$ well describes the numerical data.

We apply the scale-setting procedure discussed in Sec. III B and define the lattice spacing in units of the gradient-flow scale as $\hat{a} \equiv a / w_{0}$. All other dimensional quantities are treated accordingly, so that masses are rescaled as in $\hat{m}_{M} \equiv w_{0} m_{M}$ and decay constants as in $\hat{f}_{M}=w_{0} f_{M}$. Tree-level NLO $\mathrm{W} \chi \mathrm{PT}$ assumes that the decay constant squared $\hat{f}_{\mathrm{PS}}^{2, \mathrm{NLO}}$ is linearly dependent on both $\hat{m}_{\mathrm{PS}}^{2}$ and $\hat{a}$. We extend this assumption to all other observables as well, hence defining the ansatz

$$
\begin{gathered}
\hat{f}_{M}^{2, \mathrm{NLO}} \equiv \hat{f}_{M}^{2, \chi}\left(1+L_{f, M}^{0} \hat{m}_{\mathrm{PS}}^{2}\right)+W_{f, M}^{0} \hat{a}, \\
\hat{m}_{M}^{2, \mathrm{NLO}} \equiv \hat{m}_{M}^{2, \chi}\left(1+L_{m, M}^{0} \hat{m}_{\mathrm{PS}}^{2}\right)+W_{m, M}^{0} \hat{a},
\end{gathered}
$$

for decay constants squared and masses squared, respectively. We note that the fermion mass $m_{f}$ appearing in the standard $\mathrm{W} \chi \mathrm{PT}$ has been replaced by the pseudoscalar mass squared by using LO $\chi \mathrm{PT}$ results, according to which $\hat{m}_{\mathrm{PS}}^{2}=2 B m_{f}$. The low-energy constant $B$ could in principle be determined via a dedicated study of the fermion mass, but this would go beyond our current aims. The empirical prescription we adopt requires us to identify the largest possible region of lattice data showing evidence of the linear behavior described above and then fit the data in order to identify the additive contribution proportional to $\hat{a}$. Extrapolation to the continuum is obtained by subtracting this contribution from the lattice measurements.

\section{Quenched spectrum: Fundamental fermions}

Reference [62] reported the quenched spectrum of the lightest PS, V, and AV flavored mesons for two values of the lattice coupling, $\beta=7.62$ and 8.0, with fermions in the fundamental representation. In this section, we extend the exploration of the quenched theory in several directions. First, we consider three more values of the coupling, $\beta=7.7,7.85$, and 8.2, as mentioned in Sec. III A, aiming to perform continuum extrapolations, along the lines described in Sec. IV B. Second, in order to remove potential finite-volume effects, we restrict the bare fermion mass $m_{0}$ to ensembles that satisfy the condition $m_{\mathrm{PS}} L \geq 7.5$, in line with the results of the study with dynamical fermions [67]. Only part of the data in [62] meets this restriction, over the range of $m_{0} \in[-0.7,-0.79]$ at $\beta=7.62$, measured on the lattice with extension $48 \times 24^{3}$ - corresponding to the ensemble denoted as QB1 in Table II. For the other values of the lattice coupling we perform new calculations by using lattices with extension $60 \times 48^{3}$. The details of all the ensembles are found in Sec. III A and summarized in Table II. 

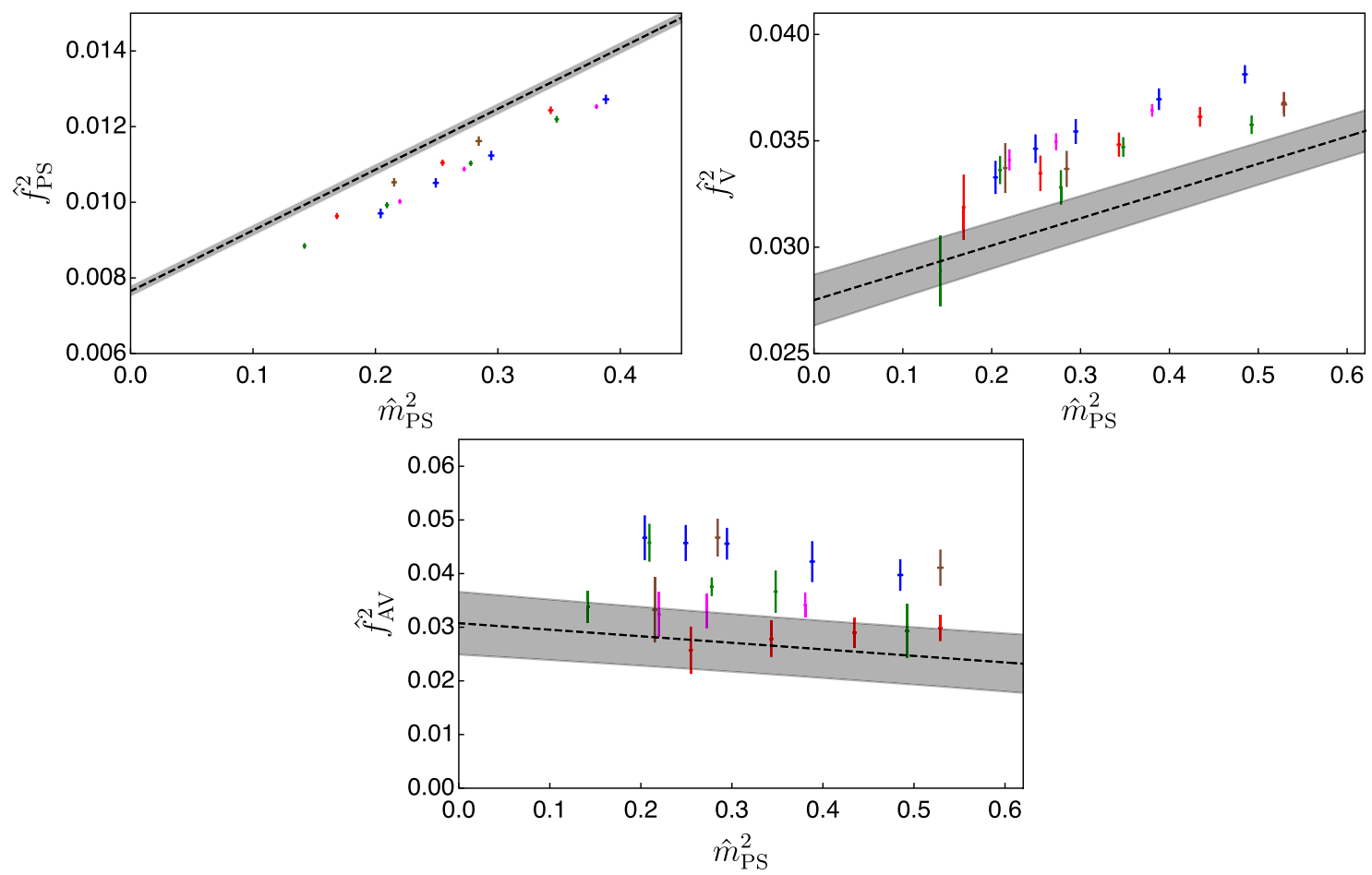

FIG. 3. Decay constants squared of PS, V, and AV mesons (constituted of fermions transforming in the fundamental representation), as a function of the PS meson mass squared $\hat{m}_{\mathrm{PS}}^{2}$, for $\beta=7.62$ (blue), 7.7 (purple), 7.85 (green), 8.0 (red), and 8.2 (brown). All quantities are expressed in units of the gradient-flow scale $w_{0}$. The results of the continuum and massless extrapolations are represented by the gray bands.

For a given ensemble $\mathrm{QB} i$ (with $i=1, \ldots, 5$ ) we introduce various choices of bare mass $a m_{0}$ of the fundamental fermions (see Table IX) and calculate two-point Euclidean correlation functions of pseudoscalar PS, vector $\mathrm{V}$, axial-vector $\mathrm{AV}$, scalar $\mathrm{S}$, (antisymmetric) tensor T and axial-tensor AT meson operators, using the interpolating operators in Table IV. We follow the standard procedure described in Sec. IVA, and we extract the masses and decay constants from the correlated fit of the data for the correlation functions as in Eq. (35). In the case of the pseudoscalar meson, we simultaneously fit the data for the correlators $C_{\mathrm{PS}, \mathrm{PS}}(t)$ and $C_{\mathrm{PS}, \mathrm{AV}}(t)$ according to Eqs. (35) and (36). The fitting intervals over the asymptotic (plateau) region at large Euclidean time are chosen to optimize the $\chi^{2}$ while keeping the interval as large as possible. Such optimized values are shown in the numerical fits presented in Tables IX and X in Appendix G.

Notice that, in the case of AV, AT and S mesons, we are not able to find an acceptable plateau region for several among the lightest choices of fermion masses. This problem appears when approximately reaching the threshold for decay to three pseudoscalars. Similar problems have been observed before in the literature on quenched theories (see for example Refs. [101-103]) and may be due to the appearance of two types of new features, both of which are ultimately due to violations of unitarity: polynomial factors correct the exponential behavior of the large-time correlation functions, and finite-volume effects do not decouple in the infinite-volume limit. We pragmatically decided to ignore measurements showing evidence of these phenomena and discard them from the analysis.

The resulting values of meson masses and decay constants are presented in Tables XIII, XIV and XVI in Appendix G. In Table XIII we also present the results of $m_{\mathrm{PS}} L$ and $f_{\mathrm{PS}} L$. For all the listed measurements the lattice volumes are large enough that the finite-volume effects are expected to be negligible as $m_{\mathrm{PS}} L \gtrsim 7.5$, and the lowenergy EFT is applicable as $f_{\mathrm{PS}} L \gtrsim 1.6$. All fermion masses are large enough that the decay of a $\mathrm{V}$ meson into two PS mesons is kinematically forbidden. The resulting values of the masses measured from the correlators involving $\mathcal{O}_{\mathrm{V}}$ and $\mathcal{O}_{\mathrm{T}}$ are statistically consistent with each other, in support of theoretical prediction: the $\mathrm{V}$ and $\mathrm{T}$ operators interpolate the same physical states with $J^{P}=1^{-}$(identified with the $\rho$ meson in the case of real-world QCD).

We perform simultaneous continuum and massless extrapolations by fitting the data for (quenched) meson masses and decay constants to Eqs. (40) and (41). We restrict the range of masses used for the extrapolations to $\hat{m}_{\mathrm{PS}}^{2} \lesssim 0.4$ for the PS states, and to ensembles yielding $\hat{m}_{\mathrm{PS}}^{2} \lesssim 0.6$ for all other states, in order to retain the largest possible range of masses within which the data show linear dependence on $\hat{m}_{\mathrm{PS}}^{2}$. In Figs. 3 and 4 we show the results of decay constants and masses, with different colors being 

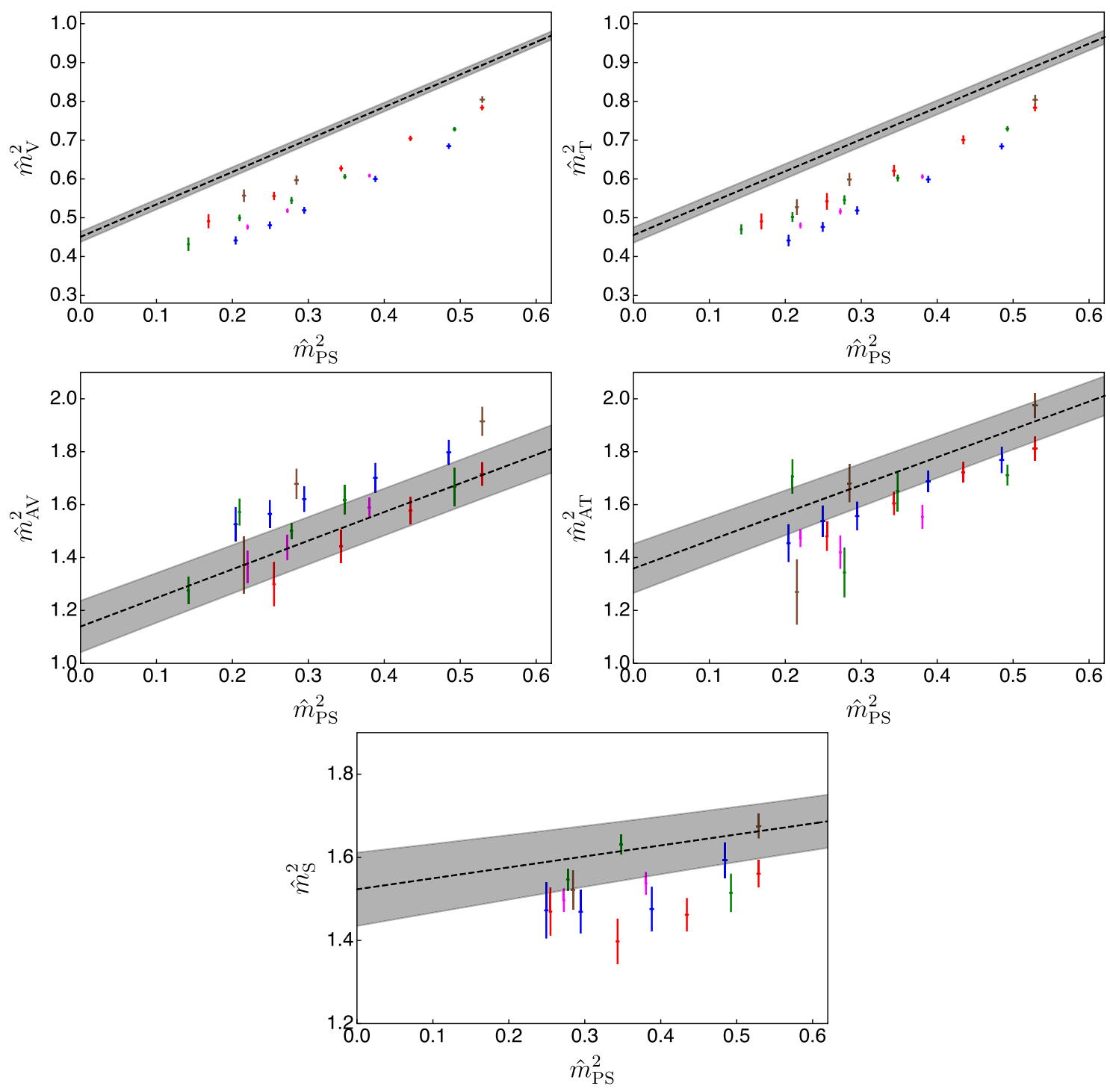

FIG. 4. Masses squared of $\mathrm{V}, \mathrm{T}, \mathrm{AV}, \mathrm{AT}$ and $\mathrm{S}$ mesons (constituted of fermions in the fundamental representation), as a function of the PS meson mass squared $\hat{m}_{\mathrm{PS}}^{2}$, for $\beta=7.62$ (blue), 7.7 (purple), 7.85 (green), 8.0 (red), and 8.2 (brown). All quantities are expressed in units of the gradient-flow scale $w_{0}$. The results of the continuum and massless extrapolations are represented by the gray bands.

used to denote ensembles at different $\beta$ values. In the figures we also present the continuum-extrapolated values (denoted by gray bands, the widths of which represent the statistical uncertainties), obtained after subtracting artifacts arising from finite lattice spacing. We find that $\hat{f}_{\mathrm{PS}}^{2}, \hat{m}_{\mathrm{V}}^{2}$ and $\hat{m}_{\mathrm{T}}^{2}$ are significantly affected by the discretization of the Euclidian space. And such a long continuum extrapolation can be understood from the fact that we have used the standard Wilson fermions. The size of lattice artifacts in all other quantities is comparable with that of statistical uncertainties.

From the numerical fits we determine the constants appearing in Eqs. (40) and (41), and we report them in Table V. The numbers in the first and second parentheses are the statistical and systematic uncertainties of the fits, respectively. In the table, we also present the values of $\chi^{2} / N_{\text {dof }}$. Some large values of $\chi^{2} / N_{\text {dof }}$ indicate that either the uncertainties of the individual data were underestimated or the fit functions are not sufficient to correctly describe the data. Although it would be difficult to fully account for the systematics associated with the continuum extrapolation with limited number of lattice spacings, we estimate the systematic uncertainties in the fits by taking the maximum and minimum values obtained from the set of data excluding the coarsest lattice (the ensemble with $\beta=7.62$ ) and including or excluding the heaviest measurements. Notice in the table that this process yields large estimates for the systematic uncertainty for those fits that result in a large value of $\chi^{2} / N_{\text {dof }}$ at the minimum. Finally, the resulting values in the massless limit, $\hat{f}_{M}^{2, \chi}$ and $\hat{m}_{M}^{2, \chi}$, should be taken with a due level of caution, since the considered masses are still relatively heavy and only the corrections corresponding to the tree-level terms in the chiral expansion are used in the 
TABLE V. Results of the fit of the coefficients in Eqs. (40) and (41), used in the continuum and massless extrapolations of masses and decay constants of mesons in the quenched simulations involving Dirac fermions in the fundamental representation. The numbers in parentheses represent, respectively, statistical and systematic uncertainties due to the fit.

\begin{tabular}{|c|c|c|c|c|}
\hline & $\hat{f}_{M}^{2, \chi}$ & $L_{f, M}^{0}$ & $W_{f, M}^{0}$ & $\chi^{2} / N_{\mathrm{dof}}$ \\
\hline PS & $0.00765(13)(11)$ & $2.101(38)(51)$ & $-0.00190(24)(15)$ & 3.1 \\
\hline $\mathrm{V}$ & $0.0275(12)(4)$ & $0.47(51)(24)$ & $0.0060(18)(4)$ & 1.5 \\
\hline \multirow[t]{2}{*}{ AV } & $0.031(6)(10)$ & $-0.40(18)(25)$ & $0.019(10)(20)$ & 4.2 \\
\hline & $\hat{m}_{M}^{2, \chi}$ & $L_{m, M}^{0}$ & $W_{m, M}^{0}$ & $\chi^{2} / N_{\mathrm{dof}}$ \\
\hline $\mathrm{V}$ & $0.451(13)(5)$ & $1.86(7)(4)$ & $-0.257(20)(6)$ & 0.4 \\
\hline $\mathrm{T}$ & $0.455(20)(7)$ & $1.81(8)(5)$ & $-0.256(31)(9)$ & 0.9 \\
\hline $\mathrm{AV}$ & $1.14(10)(14)$ & $0.96(14)(18)$ & $0.13(16)(29)$ & 3.8 \\
\hline AT & $1.36(9)(13)$ & $0.78(10)(10)$ & $-0.19(14)(24)$ & 3.1 \\
\hline $\mathrm{S}$ & $1.52(9)(4)$ & $0.18(6)(12)$ & $-0.14(13)(7)$ & 4.0 \\
\hline
\end{tabular}

fits. We leave more dedicated studies of the massless extrapolation to our future work with fully dynamical and light fermions.

As seen in Table $\mathrm{XV}$, for each given value of $\beta$ the ratio $\hat{m}_{\mathrm{V}} / \hat{f}_{\mathrm{PS}}$ is approximately constant over the mass region $\hat{m}_{\mathrm{V}} / \hat{m}_{\mathrm{PS}} \gtrsim 1.3$. From a simple linear extrapolation to the continuum of these constant vector masses in units of $\hat{f}_{\mathrm{PS}}$, we find that $\hat{m}_{\mathrm{V}} / \sqrt{2} \hat{f}_{\mathrm{PS}}=5.42(5)$. A more rigorous, yet compatible, estimate of the massless limit is obtained by taking the extrapolated results in Table $\mathrm{V}$ and yields $\hat{m}_{\mathrm{V}}^{\chi} / \sqrt{2} \hat{f}_{\mathrm{PS}}^{\chi}=5.48(9)(4)$.
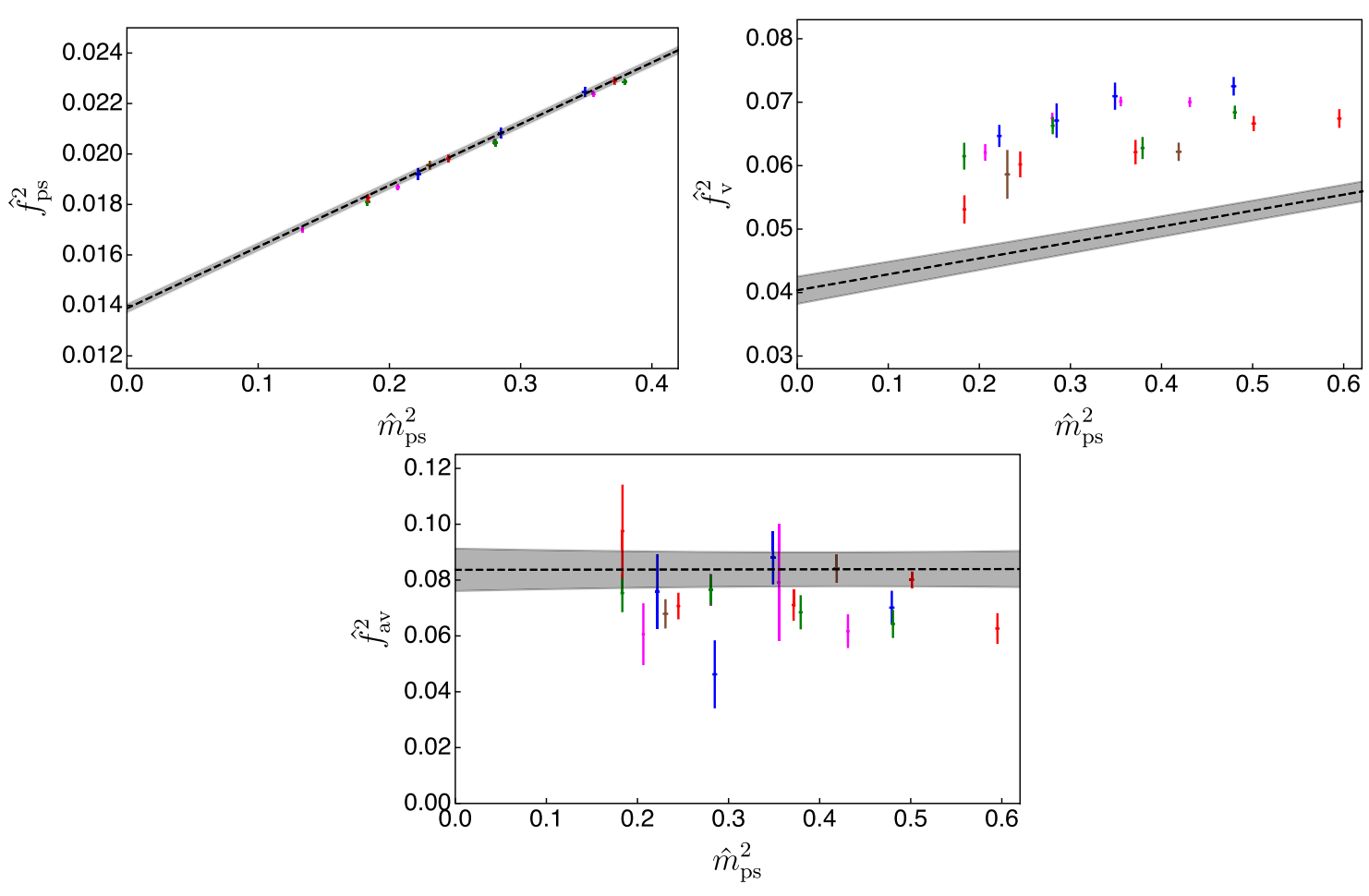

FIG. 5. Decay constants squared of ps, $v$, and av mesons (constituted of fermions in the antisymmetric representation), as a function of the ps meson mass squared $\hat{m}_{\mathrm{ps}}^{2}$, for $\beta=7.62$ (blue), 7.7 (purple), 7.85 (green), 8.0 (red), and 8.2 (brown). All quantities are expressed in units of the gradient-flow scale $w_{0}$. The results of the continuum and massless extrapolations are represented by the gray bands.

\section{Quenched spectrum: Antisymmetric fermions}

We turn now our attention to the quenched spectrum of the lightest flavored mesons involving the fermions transforming in the antisymmetric representation of $S p(4)$. We use the same ensembles listed in Table II, but the bare masses $m_{0}$ of the fermions are listed in Table XI of Appendix G. As with fundamental fermions, we choose the values of $a m_{0}$ to satisfy the condition of $m_{\mathrm{ps}} L \geq 7.5$. In the table, we also present the fitting intervals used for the extraction of the masses and the decay constants of $\mathrm{ps}, \mathrm{v}, \mathrm{av}$, and $\mathrm{s}$ mesons as well as the resulting values of $\chi^{2} / N_{\text {dof }}$. The results for $t$ and 

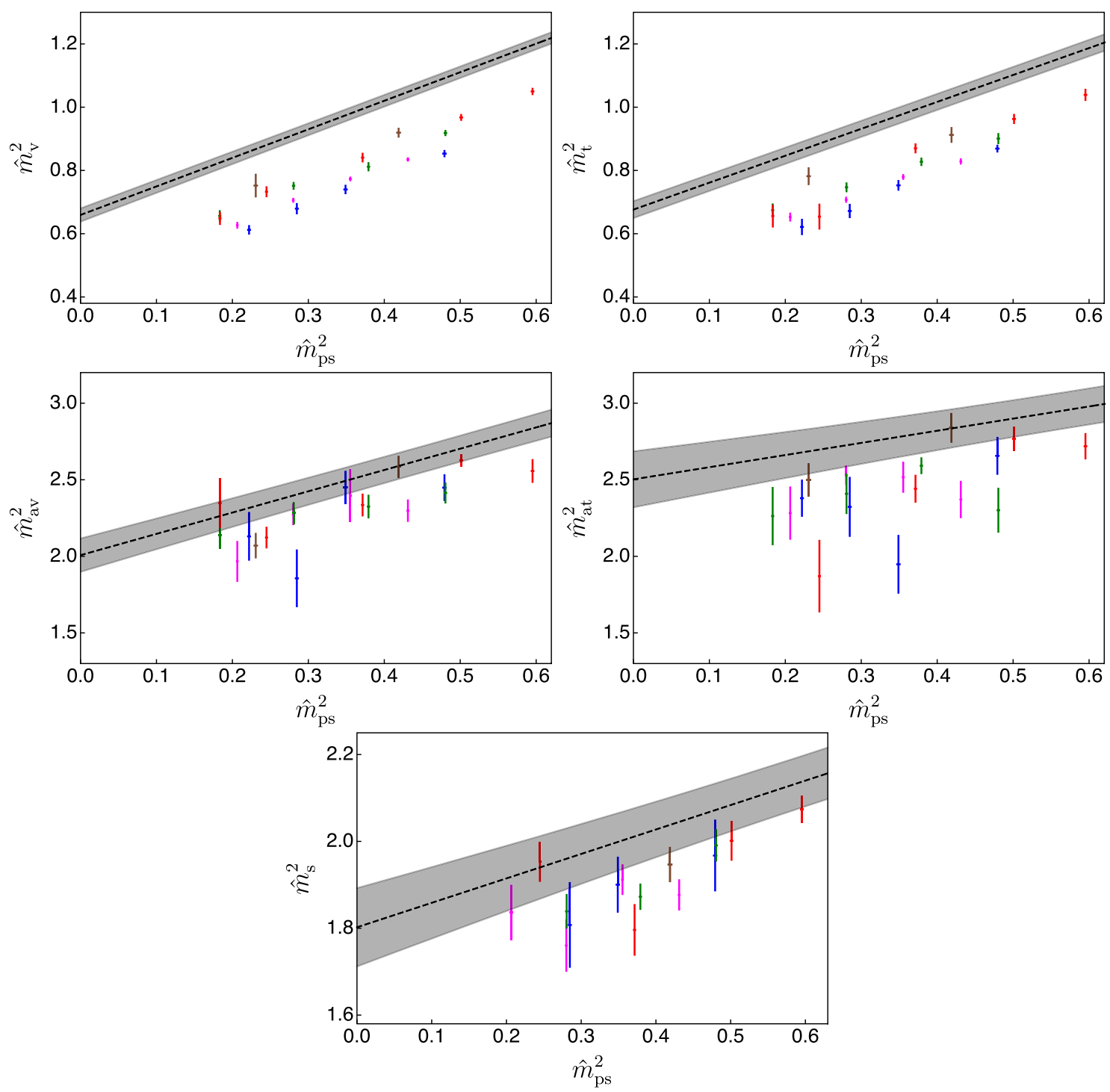

FIG. 6. Masses squared of $\mathrm{v}, \mathrm{t}, \mathrm{av}$, at and $\mathrm{s}$ mesons (constituted of fermions transforming in the antisymmetric representation), as a function of the ps meson mass squared $\hat{m}_{\mathrm{ps}}^{2}$, for $\beta=7.62$ (blue), 7.7 (purple), 7.85 (green), 8.0 (red), and 8.2 (brown). All quantities are expressed in units of the gradient-flow scale $w_{0}$. The results of the continuum and massless extrapolations are represented by the gray bands.

at mesons are shown in Table XII of the same appendix. We apply to the antisymmetric case the same numerical treatment and analysis techniques used for the fundamental fermions. As in the case of the fundamental representation, we could not find an acceptable plateau region for some measurements at the smallest fermion masses, in the cases of $\mathrm{v}$, av and s mesons.

In Appendix $\mathrm{G}$ we also present the numerical results of the masses and decay constants of ps, $\mathrm{v}$ and av mesons, as well as the masses of $\mathrm{s}, \mathrm{t}$ and at mesons. See Tables XVII, XVIII and XX. As shown in Table XVII, all the measurements meet the aforementioned condition $m_{\mathrm{ps}} L \geq 7.5$. In addition, we find that $f_{\mathrm{ps}} L \geq 2.3$, which supports the applicability of low-energy EFT techniques. Furthermore, the meson masses in units of $\hat{f}_{\mathrm{ps}}$ and the ratio $\hat{f}_{\mathrm{v}} / \hat{f}_{\mathrm{ps}}$ are presented in Tables XIX and XX. As already seen in the results for fundamental fermions, in all the measurements we find that the results of $\hat{m}_{\mathrm{t}}$ are consistent with those of $\hat{m}_{\mathrm{v}}$, given the current statistical uncertainties.

We perform the numerical fits of masses and decay constants by using the tree-level NLO $\mathrm{W} \chi \mathrm{PT}$ described by Eqs. (40) and (41). In Figs. 5 and 6, we present the fit results denoted by gray bands as well as numerical results of the masses and the decay constants measured at given lattice parameters. For the fits we consider the same ranges of $\hat{m}_{\mathrm{ps}}^{2}$ taken for the case of fundamental fermions: $\hat{m}_{\mathrm{ps}}^{2} \lesssim$ 0.4 and $\hat{m}_{\mathrm{ps}}^{2} \lesssim 0.6$, respectively, for the ps and all other states. Over these mass ranges no significant deviation from linearity of the data in $\hat{a}$ and $\hat{m}_{\mathrm{ps}}^{2}$ is visible in our data. Different colors denote different lattice couplings, while the 
TABLE VI. Results of the fit of the coefficients in Eqs. (40) and (41), used in the continuum and massless extrapolations of masses and decay constants of mesons in the quenched simulations involving Dirac fermions transforming in the 2-index antisymmetric representation. In parentheses we show statistical and systematic errors, respectively.

\begin{tabular}{|c|c|c|c|c|}
\hline & $\hat{f}_{M}^{2, \chi}$ & $L_{f, M}^{0}$ & $W_{f, M}^{0}$ & $\chi^{2} / N_{\text {dof }}$ \\
\hline ps & $0.01388(18)(10)$ & $1.754(41)(28)$ & $-0.00028(26)(15)$ & 1.3 \\
\hline $\mathrm{V}$ & $0.0404(21)(7)$ & $0.626(91)(16)$ & $0.0310(28)(17)$ & 2.4 \\
\hline \multirow[t]{2}{*}{ av } & $0.084(8)(5)$ & $0.01(13)(9)$ & $-0.022(12)(9)$ & 2.3 \\
\hline & $\hat{m}_{M}^{2, \chi}$ & $L_{m, M}^{0}$ & $W_{m, M}^{0}$ & $\chi^{2} / N_{\text {dof }}$ \\
\hline $\mathrm{v}$ & $0.657(21)(21)$ & $1.375(56)(5)$ & $-0.336(34)(4)$ & 0.8 \\
\hline $\mathrm{t}$ & $0.675(29)(19)$ & $1.26(7)(7)$ & $-0.326(47)(19)$ & 1.2 \\
\hline av & $2.01(11)(7)$ & $0.70(10)(5)$ & $-0.33(17)(11)$ & 2.1 \\
\hline at & $2.50(18)(7)$ & $0.32(12)(7)$ & $-0.48(24)(8)$ & 2.4 \\
\hline $\mathrm{s}$ & $1.80(9)(13)$ & $0.32(7)(14)$ & $-0.21(12)(15)$ & 1.6 \\
\hline
\end{tabular}

widths of the bands represent the statistical uncertainties of the continuum extrapolations.

The resulting fit values are reported in Table VI. The numbers in the first and second parentheses are the statistical and systematic uncertainties of the fits, respectively. Once more, we estimate the fitting systematics by taking the maximum and minimum values obtained from the set of data excluding the coarsest lattices at $\beta=7.62$ and including or excluding the heaviest measurements.

As in the case of fundamental fermions $Q$, we find that for each $\beta$ value the vector masses in units of the pseudoscalar decay constant are almost constant over the
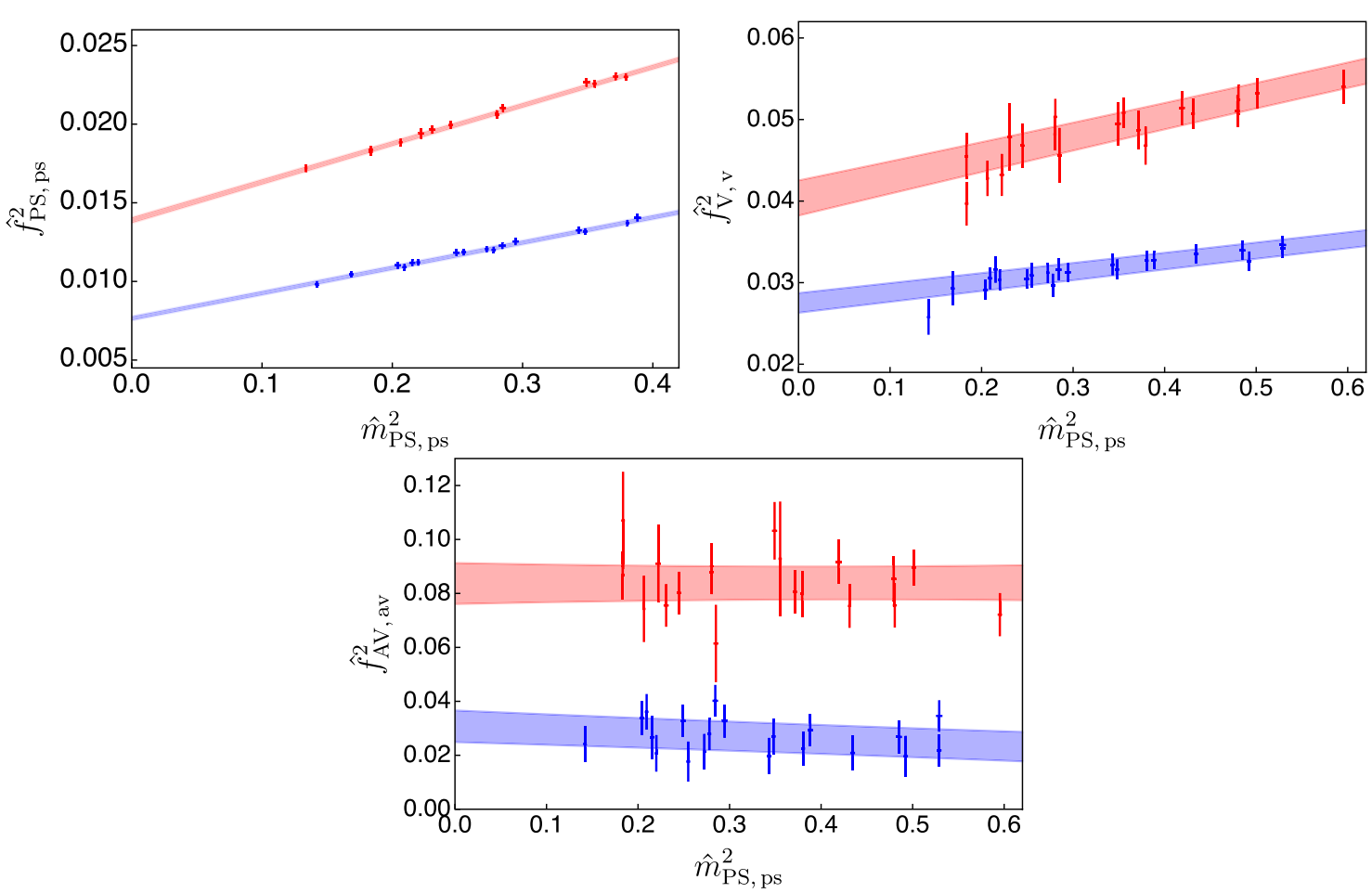

FIG. 7. Comparison of the decay constant squared (in the continuum limit) of the mesons as a function of the pseudoscalar meson mass squared, in units of $w_{0}$, for fermion constituents transforming in the fundamental (blue) or 2-index antisymmetric (red) representation. range of $\hat{m}_{\mathrm{v}} / \hat{m}_{\mathrm{ps}} \gtrsim 1.3$ - see Table XIX. After performing a simple linear extrapolation of these constants, we find that $\hat{m}_{\mathrm{v}} / \sqrt{2} \hat{f}_{\mathrm{ps}}=4.72(4)$ in the continuum limit. A more rigorous, yet compatible, estimate is obtained by making use of the extrapolated results in Table VI: we find $\hat{m}_{\mathrm{v}}^{\chi} / \sqrt{2} \hat{f}_{\mathrm{ps}}^{\chi}=4.80(12)(4)$. The resulting value of the ratio is smaller than that for the fundamental fermions by $13 \%$.

\section{E. Quenched spectrum: Comparison}

Figure 7 shows a visual comparison between the decay constants of the pseudoscalar, vector, and axial-vector 

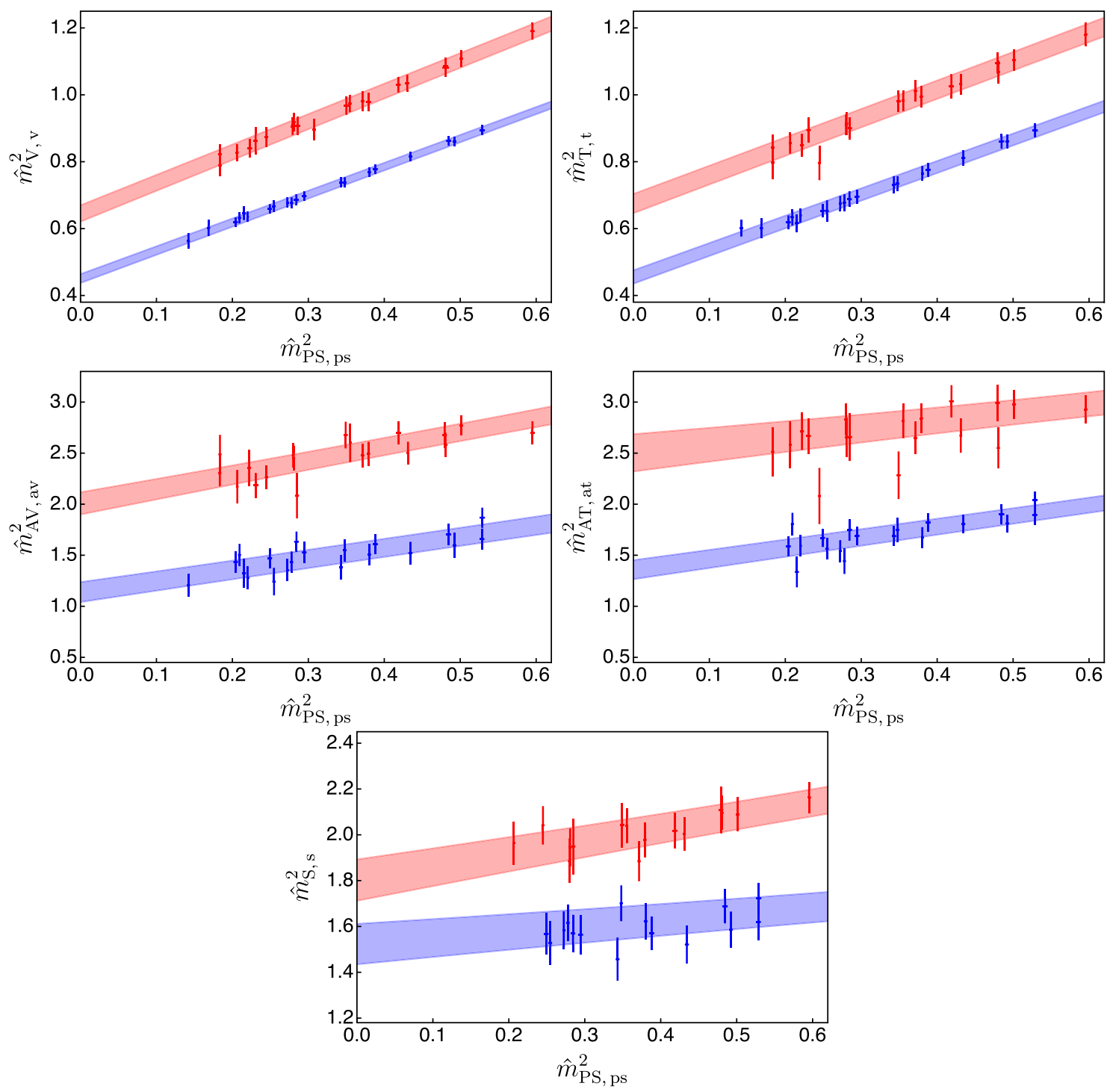

FIG. 8. Comparison of the mass squared (in the continuum limit) of the mesons as a function of the pseudoscalar meson mass squared, in units of $w_{0}$, for fermion constituents transforming in the fundamental (blue) or 2-index antisymmetric (red) representation.

mesons, made of fermions transforming in the fundamental representation of $S p(4)(\mathrm{PS}, \mathrm{V}, \mathrm{AV}$ ) and in the 2-index antisymmetric representation (ps, v, av). In order to make the comparison, we plot the continuum-limit results by naively identifying the masses of the pseudoscalars $\hat{m}_{\mathrm{PS}}^{2}=$ $\hat{m}_{\mathrm{ps}}^{2}$ as the abscissa. The comparison at finite mass should be taken with some caution, as the symmetry-breaking operators controlling the mass of PS and ps states are distinct, but the massless extrapolations can be compared unambiguously. We repeat the exercise also for the masses of all the mesons and show the result in Fig. 8.

In all cases we considered, masses and decay constants of bound states made of fermions $\Psi$ transforming in the 2index antisymmetric representation are considerably larger than those made of fermions $Q$ transforming in the fundamental representation. Focusing on the massless limit, we find that the ratio $\hat{f}_{\mathrm{av}}^{2} / \hat{f}_{\mathrm{AV}}^{2}=2.7 \pm 1.1$ is the largest, while $\hat{m}_{\mathrm{s}}^{2} / \hat{m}_{\mathrm{S}}^{2}=1.18 \pm 0.13$ is the smallest, and the other results are distributed in the range between these two values. The hierarchy between the pseudoscalar decay constants is important in the CHM context; we find that $\hat{f}_{\mathrm{ps}}^{2} / \hat{f}_{\mathrm{PS}}^{2}=1.81 \pm 0.04$. It is also to be noted that the mass of the vector states $\mathrm{v}$ is larger, but not substantially so, in respect to that of the corresponding $\mathrm{V}$ mesons, with $\hat{m}_{\mathrm{v}}^{2} / \hat{m}_{\mathrm{V}}^{2}=1.46 \pm 0.08$.

How much of the above holds true for the dynamical calculations is not known and is an interesting topic for future studies. It was shown in Ref. [67] that, by comparing quenched and dynamical calculations for mesons in the fundamental representation (performed in comparable ranges of fermion mass), and after both the continuum and massless extrapolations were performed, the discrepancies are not too large: $\mathcal{O}(25 \%)$ for $\hat{m}_{\mathrm{S}}^{2}, \mathcal{O}(20 \%)$ for $\hat{f}_{\mathrm{PS}}^{2}$, $\mathcal{O}(10 \%)$ for $\hat{m}_{\mathrm{V}}^{2}$, and smaller for the other measurements. 
Whether this is due to the fact that all the calculations in Ref. [67] are performed in a range of fermion masses that are comparatively large or to other reasons-the large- $N$ behavior of the theory might already be dominating the dynamics of $S p(4)$ mesons, for example-is not currently known and should be studied in future dedicated investigations. Yet, it is suggestive that no dramatic discrepancy has emerged so far, for all the observables we considered.

We conclude this section by reminding the reader that the calculations performed for this paper, being done with the quenched approximation, are insensitive to the number of fundamental flavors $N_{f}$ and antisymmetric flavors $n_{f}$ and hence apply to other models, beyond the phenomenologically relevant case with $N_{f}=2$ and $n_{f}=3$. A recent lattice study within the $S U(3)$ gauge theory [104] of the ratio $m_{\rho} / f_{\pi}$ between the mass of the rho mesons and the decay constant of the pions (corresponding to $m_{\mathrm{V}} / f_{\mathrm{PS}}$ in this paper) shows no appreciable dependence on the number of flavors $N_{f} \lesssim 6$-as long as the theory is deep inside the regime in which chiral symmetry breaking occurs. It would be interesting to measure whether this holds true also for other representations, in the dynamical theories. Meanwhile, we find that in our quenched calculation, after taking both the continuum and massless limits, for the fundamental representation we have $\hat{m}_{\mathrm{V}}^{2} / \hat{f}_{\mathrm{PS}}^{2}=59.0 \pm$ 2.2 , while for the antisymmetric representation we find $\hat{m}_{\mathrm{v}}^{2} / \hat{f}_{\mathrm{ps}}^{2}=47.3 \pm 2.3{ }^{6}{ }^{6}$ The discrepancy reaches beyond the $3 \sigma$ level, suggesting that this ratio-which enters into the Kawarabayashi-Suzuki-Riazuddin-Fayyazuddin (KSRF) relation $M_{\rho}^{2} /\left(g_{\rho \pi \pi}^{2} f_{\pi}^{2}\right)=2[106,107]$-depends on the fermion representation. By comparison, the ratio obtained from the numerical studies with dynamical Dirac fermions in the fundamental representation is $\hat{m}_{V}^{2} / \hat{f}_{P S}^{2}=65.4 \pm 5.0$ [67], which is slightly larger than the result of our quenched calculation. Once more, checking this result (as well as the KSRF relations) in the full dynamical theory with fermions in the antisymmetric representation would be of great interest.

\section{GLOBAL FITS}

In this section, we perform a global fit of the continuumextrapolated masses and decay constants of PS, V, and AV mesons to the EFT described in Sec. II C. As stated there, the EFT equations are applicable both to mesons constituted of fermions in the fundamental as well as 2-index antisymmetric representations of the $S p(4)$ gauge group. We also recall from Ref. [62] that several working assumptions have been used to arrive at Eqs. (19)-(24). We follow in the analysis the prescription introduced in Ref. [67]. We only repeat some of the essential features of

\footnotetext{
${ }^{6}$ These data have been used in Ref. [105] to compare these quantities with other theories.
}

the process, while referring the reader to Ref. [67] for details. We focus instead on the results of the global fit.

We start by restricting the data analyzed to lie in the mass range over which all the measured masses and decay constants can be extrapolated to the continuum limit using Eqs. (40) and (41). In the case of the fundamental representation, we restrict our measurements to include only QB1FM3-QB1FM6, QB2FM1QB2FM3, QB3FM4-QB3FM7, QB4FM6-QB4FM8, and QB5FM2-QB5FM3. In the case of antisymmetric representation, we restrict to QB1ASM4-QB1ASM6, QB2ASM3-QB2ASM6, QB3ASM2-QB3ASM4, QB4ASM4-QB4ASM6, and QB5ASM2. As anticipated in Sec. IV B, we use the $\mathrm{LO} \chi \mathrm{PT}$ result for the pseudoscalar mass and replace the fermion mass in Eqs. (19)-(24) by $\hat{m}_{\mathrm{PS}}^{2}=2 B m_{f}$. In the mass range considered, this replacement is supported by the numerical data, as $\hat{m}_{\mathrm{PS}}^{2}$ is found to be approximately linear with the mass of the fermion $\hat{m}_{0}$. Accordingly, we expand the EFT equations and truncate at the linear order in $\hat{m}_{\mathrm{PS}}^{2}$. The resulting fit equations have been presented as Eqs. (6.1)-(6.5) in Ref. [67]. The ten unknown low-energy constants, denoted as $\left(\hat{f}, \hat{F}, b, c, g_{V}, \kappa, \hat{v}_{1}, \hat{v}_{2}, \hat{y}_{3}, \hat{y}_{4}\right)$, are appropriately redefined by introducing the gradient-flow scale $w_{0}$.

We perform the numerical global fits of the data to the EFTs, via standard $\chi^{2}$ minimization, by using 200 bootstrapped samples and a simplified $\chi^{2}$ function that is built by just summing the individual $\chi^{2}$ functions for the five independent fit equations. The fit results satisfy the constraints obtained from the unitarity conditions in Eq. (6.8) of Ref. [67]. In practice, we guide the fits by an initial minimization of the full dataset. In Fig. 9 we present the results of the global fit along with the continuum-extrapolated data used for the fits, by further comparing the results originating from fundamental and antisymmetric fermions. In the figure, the fit results are presented by shaded bands, the widths of which represent the statistical uncertainties. The quality of the fits is measured by the fact that $\chi^{2} / N_{\text {dof }} \sim 0.6$ at the minimum, although one should remember that correlations have not been taken into consideration in the analysis. The results of continuum and massless extrapolations, displayed in Figs. 7 and 8, are in good agreement, even in proximity of the massless limit, with those of this alternative analysis.

As pointed out in Ref. [67], some of the parameters in the EFTs are not well constrained by the global fit of measurements coming from two-point functions only. Hence, we do not report the individual best-fit results, which are affected by flat directions and large correlations. Yet, in the same reference it is observed that some (nontrivial) combinations of the parameters may be determined well. One of the most interesting such quantities is the coupling constant associated with the decay of a vector meson $\mathrm{V}$ (or v) into two pseudoscalar mesons PS (ps). These couplings play the same role as the $g_{\rho \pi \pi}$ 

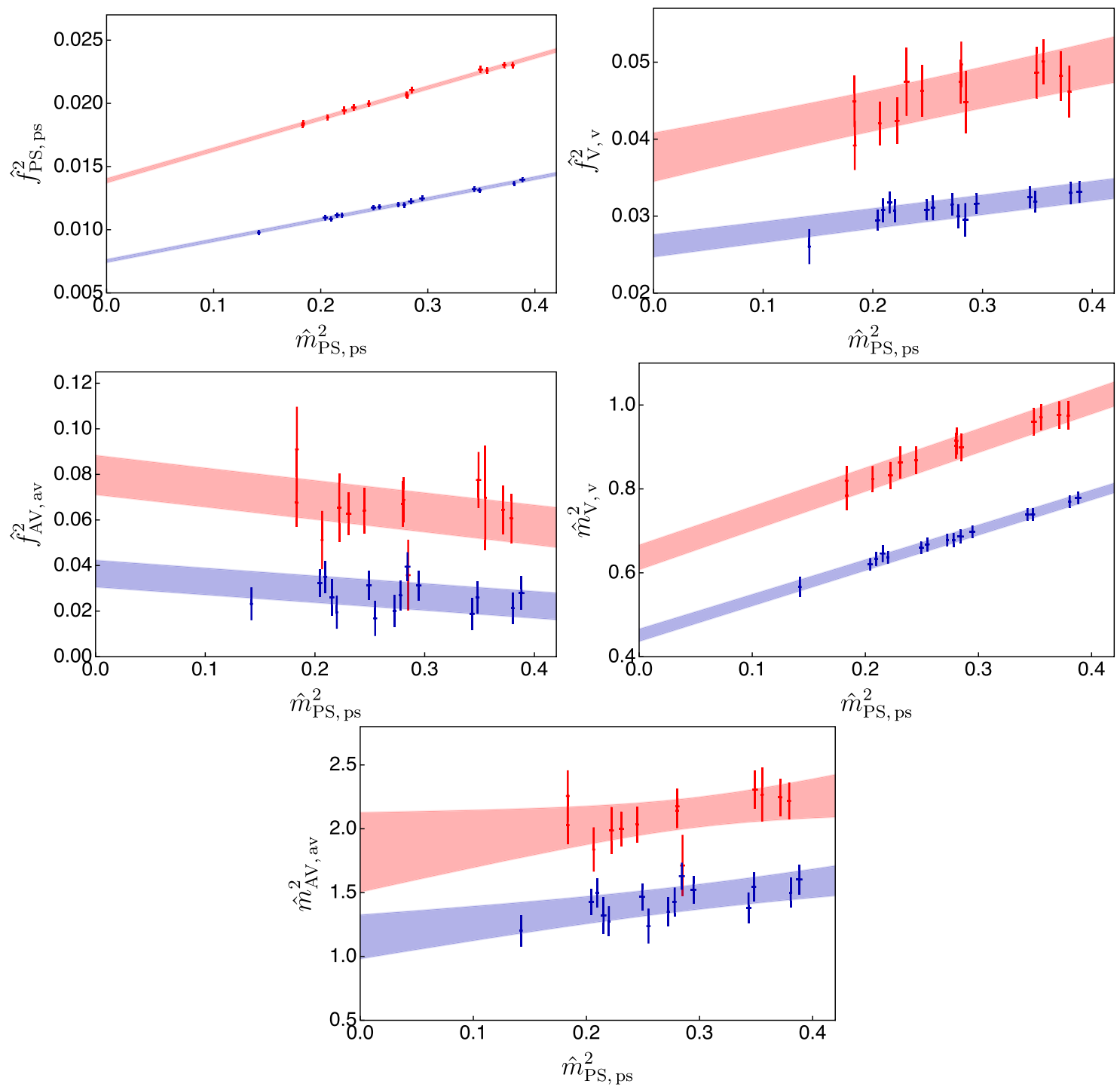

FIG. 9. Decay constants and masses in the continuum limit after subtracting lattice artifacts due to the finite lattice spacing. The global fit results are denoted by blue solid bands for the mesons constituted of fundamental fermions $Q$, and red bands for the ones constituted of antisymmetric fermions $\Psi$. The width of the bands indicates the statistical errors.

in low-energy QCD. The resulting values in the cases of fundamental and antisymmetric fermions are

$g_{\mathrm{VPP}}^{\chi}=4.95(21)(8) \quad$ and $\quad g_{\mathrm{vpp}}^{\chi}=3.80(24)(16)$,

respectively, where the suffix $\chi$ denotes the result of simultaneous continuum and massless extrapolations. As shown in Fig. 10, the distributions of this quantity exhibit a regular Gaussian shape, from which we estimate the statistical uncertainty - the numbers in the first parentheses of Eq. (42). The numbers in the second parentheses in Eq. (42) denote the systematic errors of the fits with similar caveats to those discussed in Sec. IV C, that we estimated by taking the maximum and minimum values obtained from the set of data excluding the coarsest ensemble and including or excluding the heaviest measurements.
The EFT analyses performed in this section is affected by several limitations - in particular by the quenched approximation and by the comparatively large fermion massesand thus one should interpret the results with some caution. Yet, it is interesting to compare the EFT results with phenomenological models and with available measurements obtained with dynamical fermions transforming in the fundamental representation. We first compare the EFT results in Eq. (42) with the ones predicted from the KSRF relation, $g_{\mathrm{VPP}}=m_{\mathrm{V}} / \sqrt{2} m_{\mathrm{PS}}$. We find that the left-hand side is smaller than the right-hand side of this relation by about $10 \%$ and $23 \%$, for the fundamental and antisymmetric representations, respectively. These discrepancies are larger than the uncertainties associated with the fits and might indicate that the KSRF relation does not describe the quenched theories accurately, particularly in the case of the 

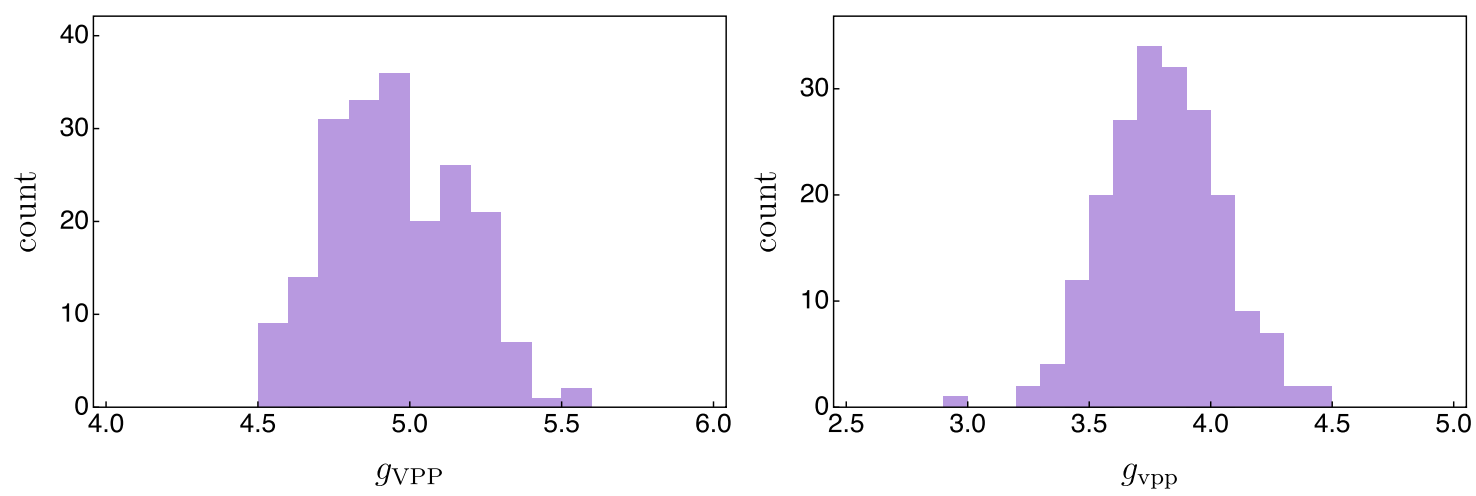

FIG. 10. Histogram distribution of the $g_{\mathrm{VPP}}$ (left panel) and $g_{\mathrm{vpp}}$ (right panel) couplings, obtained from the quenched calculation by applying the global fit strategy discussed in the text. In the former, $g_{\mathrm{VPP}}$ denotes the coupling between mesons composed of fermions in the fundamental. In the latter case the constituent fermions transform in the 2-index antisymmetric representation.

antisymmetric representation, although this statement is affected by uncontrolled systematic uncertainties due to the use of the EFT with such large values of $g_{\mathrm{VPP}}$ and $g_{\mathrm{vpp}}$, as well as large fermion masses. We also find that for the fundamental representation the quenched value of $g_{\mathrm{VPP}}^{\chi}$ is smaller by $29 \%$ compared to the dynamical value of $g_{\mathrm{VPP}}^{\chi}=$ 6.0(4)(2) [67], yielding again a discrepancy that is significantly larger than the fit uncertainties. It would be interesting to repeat these tests with dynamical fermions in the antisymmetric representation and in general to explore more directly the low-mass regimes of all these theories, but these are tasks that we leave for future extensive studies.

\section{CONCLUSIONS AND OUTLOOK}

Composite Higgs and (partial) top compositeness emerge naturally as the low-energy EFT description of gauge theories with fermion matter content in mixed representations of the gauge group. Motivated by this framework, we considered the $S p(4)$ gauge theory with $N_{f}$ quenched Wilson-Dirac fermions $Q$ transforming in the fundamental representation of $S p(4)$, as well as $n_{f}$ quenched fermions $\Psi$ in the 2-index antisymmetric representation. While the quenched theory is not expected to reproduce the full dynamics, it provides a useful comparison case for future full dynamical calculations. We generated lattice ensembles consisting of gauge configurations by means of the $\mathrm{HB}$ algorithm, modified appropriately the HIRep code [80], considered meson operators $\mathcal{O}_{M}$ bilinear in these fermions (see Table IV for explicit definitions of the operators), and measured two-point Euclidean correlation functions of such operators on discrete lattices (and in the quenched approximation).

We hence extracted decay constants $f_{M}$ and masses $m_{M}$ of the flavored mesons sourced by the operators $\mathcal{O}_{M}$, with $M=\mathrm{PS}, \mathrm{V}, \mathrm{AV}, \mathrm{S}, \mathrm{T}$, and $\mathrm{AT}$ (and $M=\mathrm{ps}, \mathrm{v}, \mathrm{av}, \mathrm{s}, \mathrm{t}$, and at), defined in Table IV. We renormalized the decay constants, expressed all dimensional quantities in terms of the gradient-flow scale $w_{0}$, and-having restricted attention to ensembles for which finite-volume effects can be ignored-applied tree-level $\mathrm{W} \chi \mathrm{PT}$ to extrapolate toward the continuum and massless limits the results for mesons constituted of both fermion species. We also performed a first global fit of the continuum results that makes use of the EFT describing the lightest spin-1 states (besides the pseudoscalars). It is constructed by extending with the language of hidden local symmetry the chiralLagrangian description of the pNGBs spanning the $S U\left(2 N_{f}\right) \times S U\left(2 n_{f}\right) / S p\left(2 N_{f}\right) \times S O\left(2 n_{f}\right)$ coset.

Our main results for the physical observables in the continuum limit are listed in the tables and plots in Secs. IV C and IVD and graphically illustrated in Sec. IVE (see in particular Figs. 7 and 8). They can be summarized as follows. In the quenched approximation, after extrapolation to the massless limit, all dimensional quantities extracted from two-point correlation functions involving operators constituted of $\Psi$ fermions are larger than the corresponding observables involving $Q$ fermions. The two extremes are $\hat{m}_{\mathrm{S}}^{2} / \hat{m}_{\mathrm{S}}^{2}=1.18 \pm 0.13$ and $\hat{f}_{\mathrm{av}}^{2} / \hat{f}_{\mathrm{AV}}^{2}=2.7 \pm$ 1.1 , respectively, with all other ratios between observables in the two sectors falling between these two values. (Of particular interest for model building are the ratios $\hat{m}_{\mathrm{v}}^{2} / \hat{m}_{\mathrm{V}}^{2}=1.46 \pm 0.08 \quad$ and $\quad \hat{f}_{\mathrm{ps}}^{2} / \hat{f}_{\mathrm{PS}}^{2}=1.81 \pm 0.04$.) The error bars comprise both statistical as well as systematic errors, the latter arising from the continuum and massless extrapolations as discussed in details in Sec. IV. Furthermore, we found statistically significant violations of the KSRF relations by the mesons made of antisymmetric fermions, at least in the quenched approximation. (The extraction of the $g_{\mathrm{VPP}}$ and $g_{\mathrm{vpp}}$ couplings from the global fit of two-point function data collected with large fermion mass to the EFT is affected by unknown systematic effects, and hence this should be taken as a preliminary result.)

Despite the physical limitations of the studied quenched theory, this paper opens the way toward addressing a number of interesting questions in future related work, a 
first class of which is related to the comparison of the quenched calculations to the full dynamical ones, in particular for the case of fermions in the antisymmetric representation. While it was observed elsewhere [67] that the quenched approximation captures remarkably well the dynamics of fundamental fermions (at least for the range of masses hitherto explored), there is no clear reason for this to happen also in the antisymmetric case, for which large- $N$ arguments are less constraining. In order to address this point, one would require to study the dynamical simulations with $\Psi$ fermions, in the phenomenologically relevant low-mass ranges of the dynamical calculations, and also to generalize our approach to $S p(2 N)$ gauge theories. The reader may be aware of the possibility that, with higherdimensional representations and large numbers of fermion degrees of freedom, some of the $\operatorname{Sp}(2 N)$ theories we are interested in might be close to the edge of the conformal window and behave very differently. [For perturbative studies within the $\operatorname{Sp}(2 N)$ class, see for instance Ref. [108-110], and references therein.]

The extensive line of research outlined in the previous paragraph complements the development of our program of studies in the context of top compositeness, that as outlined in Ref. [62] requires one to consider the dynamical theory in the presence of mixed representations. This is a novel area of exploration for lattice gauge theories, for which the literature is somewhat limited (see for instance Refs. [21,27,29,33,38]). New fermion bound states, sometimes referred to as chimera baryons, can be sourced by operators that involve gauge-invariant combinations of fermions in mixed representations. (The anomalous dimensions of chimera baryons are discussed for example in $[40,61,111]$.) The study of these states is necessary in the context of top compositeness, as they are interpreted as top partners.

A third group of future research projects can be envisioned to explore the role of higher-dimensional operators, for which the material in the Appendixes of this paper is technically useful. These operators play a role in determining the physics of vacuum (mis)alignment and of electroweak symmetry breaking, as their matrix elements enter the calculation of the potential in the low-energy EFT description. These studies would provide an additional link to phenomenological investigations of composite Higgs models, bringing lattice calculations in close contact with model-building considerations and searches for new physics at the Large Hadron Collider (LHC).

Finally, it would be interesting to investigate the finite temperature behavior of these theories. As discussed in Appendix E 1, it is important to characterize symmetry restoration and symmetry enhancement that appear at high temperatures, generalizing what has been studied about QCD to the case of real and pseudoreal representations, for which the group structure of the global symmetries and their breaking is expected to be different.

\section{ACKNOWLEDGMENTS}

We acknowledge useful discussions with Axel Maas, Roman Zwicky and Daniel Nogradi. The work of E. B., M. M., and J.R. has been funded in part by the Supercomputing Wales project, which is part funded by the European Regional Development Fund (ERDF) via Welsh Government. The work of D. K. H. was supported by Basic Science Research Program through the National Research Foundation of Korea (NRF) funded by the Ministry of Education (NRF-2017R1D1A1B06033701). The work of J.-W. L. is supported in part by the National Research Foundation of Korea grant funded by the Korea government (MSIT) (NRF-2018R1C1B3001379) and in part by Korea Research Fellowship program funded by the Ministry of Science, ICT and Future Planning through the National Research Foundation of Korea (2016H1D3A1909283). The work of C.-J. D. L. is supported by the Taiwanese MoST Grant No. 105-2628-M009-003-MY4. The work of B. L. and M.P. has been supported in part by the STFC Consolidated Grants No. ST/ L000369/1 and No. ST/P00055X/1. B. L. and M.P. received funding from the European Research Council (ERC) under the European Union's Horizon 2020 research and innovation program under Grant Agreement No. 813942. The work of B. L. is further supported in part by the Royal Society Grant No. WM170010 under the Wolfson Merit Award scheme. J. R. acknowledges support from Academy of Finland Grant No. 320123. D. V. acknowledges support from the INFN HPC-HTC project. Numerical simulations have been performed on the Swansea SUNBIRD system, on the local HPC clusters in Pusan National University (PNU) and in National ChiaoTung University (NCTU), and on the Cambridge Service for Data Driven Discovery (CSD3). The Swansea SUNBIRD system is part of the Supercomputing Wales project, which is part funded by the European Regional Development Fund (ERDF) via Welsh Government. CSD3 is operated in part by the University of Cambridge Research Computing on behalf of the STFC DiRAC HPC Facility. The DiRAC component of CSD3 was funded by BEIS capital funding via STFC capital Grants No. ST/P002307/1 and No. ST/R002452/1 and STFC operations Grant No. ST/R00689X/1. DiRAC is part of the National eInfrastructure.

\section{APPENDIX A: SPINORS}

We summarize in this Appendix our conventions in the treatment of spinors, which are useful, for example, in switching between the two-component and the four-component notation (see also Ref. [50]). The former is best suited to highlight the symmetries of the system, while the latter is the formalism adopted as a starting point for the lattice numerical treatment. We highlight some important 
symmetry aspects that offer insight in the theories studied in this paper.

For two-component spinors, we use the Pauli matrices, denoted as $\tau^{i}$, with $i=1,2,3$, and

$$
\tau^{1}=\left(\begin{array}{ll}
0 & 1 \\
1 & 0
\end{array}\right), \quad \tau^{2}=\left(\begin{array}{cc}
0 & -i \\
i & 0
\end{array}\right), \quad \tau^{3}=\left(\begin{array}{cc}
1 & 0 \\
0 & -1
\end{array}\right) .
$$

Given a two-component spinor $u$, with no internal quantum numbers, we define the $\mathcal{C}$-conjugate $u_{C} \equiv i \tau^{2} u^{*} \equiv-\tilde{C} u^{*}$. Furthermore, we introduce the notation $\sigma^{\mu} \equiv\left(\mathbb{1}_{2},-\tau^{i}\right)$ and $\bar{\sigma}^{\mu} \equiv\left(\mathbb{1}_{2}, \tau^{i}\right)$.

We adopt conventions in which the space-time Minkowski metric is

$$
\eta_{\mu \nu} \equiv\left(\begin{array}{cccc}
1 & & & \\
& -1 & & \\
& & -1 & \\
& & & -1
\end{array}\right)=\eta^{\mu \nu} .
$$

The Dirac algebra is defined by the anticommutation relation

$$
\left\{\gamma^{\mu}, \gamma^{\nu}\right\}=2 \eta^{\mu \nu},
$$

with the $4 \times 4$ matrix $\gamma^{0}$ Hermitian, while the three $\gamma^{i}$ are anti-Hermitian, so that $\gamma^{0} \gamma^{\mu} \gamma^{0}=\gamma^{\mu \dagger}$. Chirality is defined by the eigenvalues of the matrix $\gamma_{5} \equiv i \gamma^{0} \gamma^{1} \gamma^{2} \gamma^{3}$, that satisfies the relation $\left\{\gamma^{\mu}, \gamma^{5}\right\}=0$.

The charge-conjugation matrix $C=i \gamma^{2} \gamma^{0}$ obeys the defining relations $C \gamma^{\mu} C^{-1}=-\gamma^{\mu \mathrm{T}}$ and $C^{2}=-\mathbb{1}_{4}=-C C^{\dagger}$. The chiral representation of the $\gamma^{\mu}$ matrices is

$$
\begin{array}{ll}
\gamma^{0}=\left(\begin{array}{cc}
0 & \mathbb{1}_{2} \\
\mathbb{1}_{2} & 0
\end{array}\right), & \gamma^{i}=\left(\begin{array}{cc}
0 & -\tau^{i} \\
\tau^{i} & 0
\end{array}\right), \\
\gamma^{5}=\left(\begin{array}{cc}
\mathbb{1}_{2} & 0 \\
0 & -\mathbb{1}_{2}
\end{array}\right), & C=\left(\begin{array}{cc}
-i \tau^{2} & 0 \\
0 & i \tau^{2}
\end{array}\right),
\end{array}
$$

which implies the useful relations

$$
\gamma^{0} \gamma^{\mu}=\left(\begin{array}{cc}
\bar{\sigma}^{\mu} & 0 \\
0 & \sigma^{\mu}
\end{array}\right), \quad C \gamma^{0} \gamma^{\mu} C^{-1}=\left(\begin{array}{cc}
\sigma^{\mu} & 0 \\
0 & \bar{\sigma}^{\mu}
\end{array}\right) .
$$

We also define the matrices

$$
\sigma^{\mu \nu} \equiv \frac{i}{2}\left[\gamma^{\mu}, \gamma^{\nu}\right],
$$

which obey the relations $\left[\gamma_{5}, \sigma^{\mu \nu}\right]=0, \gamma^{0} \sigma^{\mu \nu} \gamma^{0}=\left(\sigma^{\mu \nu}\right)^{\dagger}$ and $\gamma_{5} \sigma^{\mu \nu}=\frac{i}{2} \epsilon^{\mu \nu \rho \sigma} \sigma_{\rho \sigma}$, where $\epsilon^{\mu \nu \rho \sigma}$ is the completely antisymmetric Levi-Civita symbol. In the chiral representation for the $\gamma^{\mu}$ matrices, the six $\sigma^{\mu \nu}$ matrices are block diagonal and can be written as $\sigma^{\mu \nu}=\left(\begin{array}{cc}\sigma_{L L}^{\mu \nu} & 0 \\ 0 & \sigma_{R R}^{\mu \nu}\end{array}\right), \quad \sigma^{\mu \nu} \gamma_{5}=\left(\begin{array}{cc}\sigma_{L L}^{\mu \nu} & 0 \\ 0 & -\sigma_{R R}^{\mu \nu}\end{array}\right)=\gamma_{5} \sigma^{\mu \nu}$.

By isolating the spatial indices $i$, one finds that

$$
\sigma^{0 i}=i\left(\begin{array}{cc}
\tau^{i} & 0 \\
0 & -\tau^{i}
\end{array}\right), \quad \sigma^{i j}=\epsilon^{i j k}\left(\begin{array}{cc}
\tau^{k} & 0 \\
0 & \tau^{k}
\end{array}\right)
$$

We introduce the notation $\bar{\lambda} \equiv \lambda^{\dagger} \gamma^{0}$. A single Majorana spinor $\lambda$ obeys the relation $\lambda= \pm \lambda_{C} \equiv \pm C \bar{\lambda}^{\mathrm{T}} \equiv \pm C \gamma^{0} \lambda^{*}=$ $\pm i \gamma^{2} \lambda^{*}$. We conventionally resolve the \pm ambiguity by the choice of the + sign. Starting from a two-component spinor $u$, a four-component Majorana spinor is

$$
\lambda=\left(\begin{array}{c}
u \\
i \tau^{2} u^{*} \equiv-\tilde{C} u^{*}
\end{array}\right),
$$

so that $\lambda=\lambda_{C}$. The left-handed (LH) chiral projector is $P_{L}=\frac{1}{2}\left(\mathbb{1}_{4}+\gamma_{5}\right)$, so that a four-component LH chiral spinor $\lambda_{L}=P_{L} \lambda$ satisfies $P_{L} \lambda_{L}=\lambda_{L}$. Analogous definitions apply to the right-handed (RH) projector $P_{R}$ and spinor $\lambda_{R}$. The decomposition in LH and RH four-component chiral Weyl spinors is given by

$$
\lambda_{L}=\left(\begin{array}{l}
u \\
0
\end{array}\right), \quad \lambda_{R}=\left(\begin{array}{c}
0 \\
i \tau^{2} u^{*} \equiv-\tilde{C} u^{*}
\end{array}\right)
$$

and yields the relations $\lambda_{L}=C \overline{\lambda_{R}}$ and $\overline{\lambda_{L}}=\lambda_{R}^{\mathrm{T}} C=-\lambda_{R}^{\mathrm{T}} C^{-1}$. Clearly, $u, \lambda, \lambda_{L}$ and $\lambda_{R}$ are different ways to encode the same information.

Consider two distinct, two-component spinors $u$ and $d$, with no additional internal degrees of freedom (aside from the spinor index $\alpha=1,2$ ). When taken together, they naturally define the fundamental representation of a global $U(2)$ symmetry. Their components are described by Grassmann variables, satisfying the two nontrivial relations ${ }^{7}$

$$
u^{\alpha *} d^{\beta}=-d^{\beta} u^{\alpha *}, \quad\left(u^{\alpha *} d^{\beta}\right)^{*}=d^{\beta *} u^{\alpha},
$$

and analogous for all other combinations.

A Dirac four-component spinor is obtained by joining the LH projection of the Majorana spinor built starting from $u$ and the RH projection of the Majorana spinor corresponding to $d$, so that $Q=U_{L}+D_{R}$ with

\footnotetext{
${ }^{7}$ The first one is the defining relation of the anticommuting Grassmann variable, while the second is required for consistency of the definition of absolute value as a real number $\xi^{*} \xi=\left(\xi^{*} \xi\right)^{*} \neq 0$.
} 


$$
U_{L} \equiv\left(\begin{array}{l}
u \\
0
\end{array}\right), \quad D_{R} \equiv\left(\begin{array}{c}
0 \\
-\tilde{C} d^{*}
\end{array}\right), \quad D_{L} \equiv\left(\begin{array}{l}
d \\
0
\end{array}\right), \quad U_{R} \equiv\left(\begin{array}{c}
0 \\
-\tilde{C} u^{*}
\end{array}\right)
$$

and $Q_{C} \equiv C \bar{Q}^{\mathrm{T}}=C \gamma^{0} Q^{*}=D_{L}+U_{R}$, while $\overline{Q_{C}}=Q^{\mathrm{T}} C$.

By inspection, one finds that the following relations hold true:

$$
\begin{aligned}
\bar{Q} P_{L} Q & =\overline{D_{R}} U_{L}=d^{\mathrm{T}} \tilde{C} u, & & \left(\bar{Q} P_{L} Q\right)^{*}=\bar{Q} P_{R} Q=-u^{\dagger} \tilde{C} d^{*}, \\
\overline{Q_{C}} P_{L} Q_{C} & =\overline{U_{R}} D_{L}=u^{\mathrm{T}} \tilde{C} d, & & \left(\overline{Q_{C}} P_{L} Q_{C}\right)^{*}=\overline{Q_{C}} P_{R} Q_{C}=-d^{\dagger} \tilde{C} u^{*}, \\
\bar{Q} P_{L} Q_{C} & =\overline{D_{R}} D_{L}=d^{\mathrm{T}} \tilde{C} d, & & \left(\bar{Q} P_{L} Q_{C}\right)^{*}=\overline{Q_{C}} P_{R} Q=-d^{\dagger} \tilde{C} d^{*}, \\
\overline{Q_{C}} P_{L} Q & =\overline{U_{R}} U_{L}=u^{\mathrm{T}} \tilde{C} u, & & \left(\overline{Q_{C}} P_{L} Q\right)^{*}=\bar{Q} P_{R} Q_{C}=-u^{\dagger} \tilde{C} u^{*},
\end{aligned}
$$

and by using the $\gamma^{\mu}$ matrices, one also finds the relations

$$
\begin{aligned}
\bar{Q} \gamma^{\mu} P_{L} Q & =\overline{U_{L}} \gamma^{\mu} U_{L}=u^{\dagger} \bar{\sigma}^{\mu} u, & & \left(\bar{Q} \gamma^{\mu} P_{L} Q\right)^{\mathrm{T}}=-\overline{Q_{C}} \gamma^{\mu} P_{R} Q_{C}=-u^{\mathrm{T}} \bar{\sigma}^{\mu *} u^{*}, \\
\overline{Q_{C}} \gamma^{\mu} P_{L} Q_{C} & =\overline{D_{L}} \gamma^{\mu} D_{L}=d^{\dagger} \bar{\sigma}^{\mu} d, & & \left(\overline{Q_{C}} \gamma^{\mu} P_{L} Q_{C}\right)^{\mathrm{T}}=-\bar{Q} \gamma^{\mu} P_{R} Q=-d^{\mathrm{T}} \bar{\sigma}^{\mu *} d^{*}, \\
\bar{Q} \gamma^{\mu} P_{L} Q_{C} & =\overline{U_{L}} \gamma^{\mu} D_{L}=u^{\dagger} \bar{\sigma}^{\mu} d, & & \left(\bar{Q} \gamma^{\mu} P_{L} Q_{C}\right)^{\mathrm{T}}=-\bar{Q} \gamma^{\mu} P_{R} Q_{C}=-d^{\mathrm{T}} \bar{\sigma}^{\mu *} u^{*}, \\
\overline{Q_{C}} \gamma^{\mu} P_{L} Q & =\overline{D_{L}} \gamma^{\mu} U_{L}=d^{\dagger} \bar{\sigma}^{\mu} u, & & \left(\overline{Q_{C}} \gamma^{\mu} P_{L} Q\right)^{\mathrm{T}}=-\overline{Q_{C}} \gamma^{\mu} P_{R} Q=-u^{\mathrm{T}} \bar{\sigma}^{\mu *} d^{*},
\end{aligned}
$$

as well as

$$
\begin{aligned}
\left(\bar{Q} \gamma^{\mu} P_{L} Q\right)^{*} & =\bar{Q} \gamma^{\mu} P_{L} Q, \\
\left(\overline{Q_{C}} \gamma^{\mu} P_{L} Q_{C}\right)^{*} & =\overline{Q_{C}} \gamma^{\mu} P_{L} Q_{C}, \\
\left(\bar{Q} \gamma^{\mu} P_{L} Q_{C}\right)^{*} & =\overline{Q_{C}} \gamma^{\mu} P_{L} Q, \\
\left(\overline{Q_{C}} \gamma^{\mu} P_{L} Q\right)^{*} & =\bar{Q} \gamma^{\mu} P_{L} Q_{C} .
\end{aligned}
$$

By definition, the transpose of a $\mathbb{C}$ number is trivial, and hence $\xi A \chi=(\xi A \chi)^{\mathrm{T}} \equiv-\chi^{\mathrm{T}} A^{\mathrm{T}} \xi^{\mathrm{T}}$, for any $\xi, \chi$ spinor written in terms of Grassmann variables and $A$ any matrix of $\mathbb{C}$ numbers. This implies the relation

$$
\bar{Q} P_{L} Q-\overline{Q_{C}} P_{L} Q_{C}=d^{\mathrm{T}} \tilde{C} u-u^{\mathrm{T}} \tilde{C} d=0,
$$

which will be useful later. Some algebra shows that the following identity between real numbers holds:

$$
\begin{aligned}
& \frac{1}{2}\left(i \bar{Q} \gamma^{\mu} \partial_{\mu} Q-i \overline{\partial_{\mu} Q} \gamma^{\mu} Q\right) \\
& \quad=\frac{1}{2} \sum_{j=1}^{2}\left(i q^{j \dagger} \bar{\sigma}^{\mu} \partial_{\mu} q^{j}-i \partial_{\mu} q^{j \dagger} \bar{\sigma}^{\mu} q^{j}\right),
\end{aligned}
$$

where $q^{j}=(u, d)$ and where the $U(2)=U(1) \times S U(2)$ global symmetry is now made manifest. This is adopted as the kinetic term of the Dirac spinor $Q$.

The Lagrangian density for the Dirac spinor $Q$ admits also a mass term. By virtue of the relations $\tilde{C}^{\dagger}=-\tilde{C}=\tilde{C}^{\mathrm{T}}$, and by the Grassmann nature of the spinors, it can be written in terms of the symmetric matrix $\omega \equiv \tau^{1}$ :

$$
\begin{aligned}
-\mathcal{M} \bar{Q} Q & =-\mathcal{M}\left(\overline{U_{L}} D_{R}+\overline{D_{R}} U_{L}\right) \\
& =-\mathcal{M}\left(-u^{\dagger} \tilde{C} d^{*}+d^{\mathrm{T}} \tilde{C} u\right) \\
& =-\frac{1}{2} \mathcal{M}\left(-u^{\dagger} \tilde{C} d^{*}-d^{\dagger} \tilde{C} u^{*}+u^{\mathrm{T}} \tilde{C} d+d^{\mathrm{T}} \tilde{C} u\right) \\
& =-\frac{1}{2} \mathcal{M} \sum_{j k} \omega_{j k}\left(q^{j \mathrm{~T}} \tilde{C} q^{k}-q^{j \dagger} \tilde{C} q^{k *}\right) .
\end{aligned}
$$

This term breaks the symmetry to the subgroup $O(2) \in$ $U(2){ }^{8}$

The real Lagrangian density of a single Dirac fermion is then

$$
\begin{aligned}
\mathcal{L}= & \frac{1}{2}\left(i \bar{Q} \gamma^{\mu} \partial_{\mu} Q-i \overline{\partial_{\mu} Q} \gamma^{\mu} Q\right)-\mathcal{M} \bar{Q} Q \\
= & \bar{Q}\left(i \gamma^{\mu} \partial_{\mu}-\mathcal{M}\right) Q+\partial_{\mu}(\ldots) \\
= & \frac{1}{2} \sum_{j}\left(i q^{j \dagger} \bar{\sigma}^{\mu} \partial_{\mu} q^{j}-i \partial_{\mu} q^{j \dagger} \bar{\sigma}^{\mu} q^{j}\right) \\
& -\frac{1}{2} \mathcal{M} \sum_{j k} \omega_{j k}\left(q^{j \mathrm{~T}} \tilde{C} q^{k}-q^{j \dagger} \tilde{C} q^{k *}\right),
\end{aligned}
$$

\footnotetext{
${ }^{8}$ If the spinors have additional, internal degrees of freedom, their anticommuting nature, which ultimately descends from Fermi-Dirac statistics, might enforce to antisymmetrize over them and can lead to the replacement of the symmetric $\omega$ with an antisymmetric $\Omega$. Such is indeed the case if $Q$ transforms in the fundamental of $\operatorname{Sp}(2 N)$, for example. Alternatively, if one has to antisymmetrize in two gauge indices, as in the case discussed in Ref. [112] and also in the case relevant to the $\Psi$ spinors on the antisymmetric 2-index representation, symmetry breaking is, once more, controlled by the symmetric matrix $\omega$.
} 
the first line of which (by ignoring the surface term) yields the Dirac equation:

$$
\left(i \gamma^{\mu} \partial_{\mu}-\mathcal{M}\right) Q=0 .
$$

Equation (A21) can be generalized by adding a symmetric $\mathcal{M}_{j k}$ Majorana mass matrix via the replacement $\mathcal{M} \omega_{j k} \rightarrow \mathcal{M}_{j k}$ in the two-component formulation:

$$
\begin{aligned}
2 \mathcal{L}_{M}^{\prime}= & \sum_{j}\left(i q^{j \dagger} \bar{\sigma}^{\mu} \partial_{\mu} q^{j}-i \partial_{\mu} q^{j \dagger} \bar{\sigma}^{\mu} q^{j}\right) \\
& -\sum_{j k}\left(\mathcal{M}_{j k} q^{j \mathrm{~T}} \tilde{C} q^{k}-\mathcal{M}_{j k}^{*} q^{j \dagger} \tilde{C} q^{k *}\right) .
\end{aligned}
$$

The Majorana mass term can then be written also in terms of four-component Dirac spinors by applying the projector $P_{L}$ and $C$ along the lines of Eq. (A13), as follows:

$\mathcal{L}^{\prime}=-\frac{1}{2}\left(\left(P_{L} Q\right)^{\mathrm{T}},\left(P_{L} Q_{C}\right)^{\mathrm{T}}\right) C \mathcal{M}\left(\begin{array}{c}P_{L} Q \\ P_{L} Q_{C}\end{array}\right)+$ H.c.,

where the matrix $\mathcal{M}$ is defined as

$\mathcal{M}=\left(\begin{array}{cc}\mathcal{M}_{u u} & \mathcal{M}_{u d}=\frac{1}{2}\left(\mathcal{M}_{s}+\mathcal{M}_{a}\right) \\ \mathcal{M}_{d u}=\frac{1}{2}\left(\mathcal{M}_{s}-\mathcal{M}_{a}\right) & \mathcal{M}_{d d}\end{array}\right)$.

If there are no other internal degrees of freedom, $\mathcal{M}$ is symmetric, with $\mathcal{M}_{u d}=\mathcal{M}_{d u}$. In the language of $U(2)$, the product of two doublets naturally decomposes as $3 \oplus 1$ of $U(2)$ :

$3 \sim\left(\begin{array}{c}\overline{Q_{C}} P_{L} Q=u^{\mathrm{T}} \tilde{C} u \\ \frac{1}{2}\left(\bar{Q} P_{L} Q+\overline{Q_{C}} P_{L} Q_{C}\right)=\frac{1}{2}\left(d^{\mathrm{T}} \tilde{C} u+u^{\mathrm{T}} \tilde{C} d\right) \\ \bar{Q} P_{L} Q_{C}=d^{\mathrm{T}} \tilde{C} d\end{array}\right)$,

$1 \sim \frac{1}{2}\left(\bar{Q} P_{L} Q-\bar{Q}_{C} P_{L} Q_{C}\right)=\frac{1}{2}\left(d^{\mathrm{T}} \tilde{C} u-u^{\mathrm{T}} \tilde{C} d\right)$.

The latter vanishes in the absence of additional degrees of freedom, due to Eq. (A17).

\section{APPENDIX B: A NOTE ABOUT MASSIVE VECTORS}

A massive vector of mass $m$ in $D=4$ space-time dimensions can be described by two equivalent quantum theories, with different field content and Lagrangian densities (see for instance the detailed discussions in Refs. [113-116], and references therein).

(i) A vector field $A_{\mu}$ couples to a scalar field $\pi$, with Lagrangian density

$$
\mathcal{L}_{0}=-\frac{1}{4} F_{\mu \nu} F^{\mu \nu}-\frac{1}{2}\left(\partial_{\mu} \pi+m A_{\mu}\right)\left(\partial^{\mu} \pi+m A^{\mu}\right),
$$

where $F_{\mu \nu}=\partial_{\mu} A_{\nu}-\partial_{\nu} A_{\mu} \cdot \mathcal{L}_{0}$ is invariant under the gauge transformations

$$
\pi \rightarrow \pi+m \alpha, \quad A_{\mu} \rightarrow A_{\mu}-\partial_{\mu} \alpha,
$$

with $\alpha=\alpha(x)$. The gauge choice $\alpha=-\pi / m$ removes $\pi$ from the Lagrangian density, which then depends only on a massive vector field.

(ii) A 2-index antisymmetric form $\mathcal{B}_{\mu \nu}$ is coupled to a vector $\mathcal{A}_{\mu}$ (not to be confused with $A_{\mu}$ ), and the Lagrangian density is

$$
\mathcal{L}_{1}=-\frac{1}{12} G_{\mu \nu \rho} G^{\mu \nu \rho}-\frac{1}{4} \mathcal{H}_{\mu \nu} \mathcal{H}^{\mu \nu}
$$

where $\quad \mathcal{F}_{\mu \nu} \equiv \partial_{\mu} \mathcal{A}_{\nu}-\partial_{\nu} \mathcal{A}_{\mu}, \quad \mathcal{H}_{\mu \nu} \equiv \mathcal{F}_{\mu \nu}+m \mathcal{B}_{\mu \nu}$ and $G_{\mu \nu \rho} \equiv \partial_{\mu} \mathcal{B}_{\nu \rho}+\partial_{\rho} \mathcal{B}_{\mu \nu}+\partial_{\nu} \mathcal{B}_{\rho \mu}$. The Lagrangian $\mathcal{L}_{1}$ is invariant under the gauge transformation

$$
\mathcal{A}_{\mu} \rightarrow \mathcal{A}_{\mu}+m \alpha_{\mu}, \quad \mathcal{B}_{\mu \nu} \rightarrow \mathcal{B}_{\mu \nu}-\partial_{\mu} \alpha_{\nu}+\partial_{\nu} \alpha_{\mu},
$$

with the vector $\alpha_{\mu}=\alpha_{\mu}(x)$. The gauge choice $\alpha_{\mu}=$ $-\mathcal{A}_{\mu} / m$ removes $\mathcal{A}_{\mu}$ from the Lagrangian density, which then depends only on a massive 2-form field. The Lagrangian $\mathcal{L}_{1}$ can also be rewritten, by defining $\mathcal{K}_{\mu \nu} \equiv \frac{1}{2 m} \epsilon_{\mu \nu \rho \sigma} \mathcal{H}^{\rho \sigma}$, in the form

$$
\mathcal{L}_{1}=\frac{1}{2} \partial^{\alpha} \mathcal{K}_{\mu \alpha} \partial^{\beta} \mathcal{K}_{\beta}^{\mu}+\frac{m^{2}}{4} \mathcal{K}_{\mu \nu} \mathcal{K}^{\mu \nu}
$$

Gauge invariance is not manifest in this form. The Lagrangians $\mathcal{L}_{0}$ and $\mathcal{L}_{1}$ are equivalent at the level of the path integrals they define [113-116]. Hence, the use of antisymmetric massive 2-index tensors provides an alternative, equivalent descriptions of massive vectors.

In physical terms, there is no difference between these two (or rather, three) formulations. Important differences are introduced by the coupling to matter fields and sources. For example, one can couple fermions to $A_{\mu}$ via the new term

$$
\mathcal{L}_{A}=i g \bar{Q} \gamma^{\mu} A_{\mu} P_{L} Q,
$$

with $Q$ a Dirac fermion and $g$ the coupling. For the antisymmetric tensor, one may write

$$
\mathcal{L}_{\mathcal{B}}=g \bar{Q} \sigma^{\mu \nu} \mathcal{B}_{\mu \nu} P_{L} Q .
$$

While $\mathcal{L}_{A}$ couples the spin-1 field to the LH component only of $Q$, in $\mathcal{L}_{\mathcal{B}}$ the $\mathrm{LH}$ and $\mathrm{RH}$ projections are coupled to one another, so that while $\mathcal{L}_{0}$ and $\mathcal{L}_{1}$ in isolation define the 
same theory, the addition of $\mathcal{L}_{\mathcal{A}}$ or $\mathcal{L}_{\mathcal{B}}$ leaves different global symmetries and different coupled theories.

\section{APPENDIX C: ABOUT LIE GROUPS, ALGEBRAS AND SM EMBEDDING}

Here we summarize some group theory notions relevant for models of composite Higgs and top quark compositeness based on the $S U(4) / S p(4) \otimes S U(6) / S O(6)$ coset $[12,43]$. We do not repeat unnecessary details-in particular, our special choice of $S U(4)$ generators can be found elsewhere [50] - but we explicitly show the embedding of the SM gauge group (and fields, when useful).

The $S U(4) / S p(4)$ coset governs the Higgs sector of the Standard Model. Given the form of $\Omega$ in Eq. (5), the unbroken subgroup $S O(4) \sim S U(2)_{L} \times S U(2)_{R}$ is the subset of the unbroken global $S p(4) \subset S U(4)$ that is generated by the following elements of the associated algebra:

$$
\begin{aligned}
& T_{L}^{1}=\frac{1}{2}\left(\begin{array}{cccc}
0 & 0 & 1 & 0 \\
0 & 0 & 0 & 0 \\
1 & 0 & 0 & 0 \\
0 & 0 & 0 & 0
\end{array}\right), \quad T_{L}^{2}=\frac{1}{2}\left(\begin{array}{cccc}
0 & 0 & -i & 0 \\
0 & 0 & 0 & 0 \\
i & 0 & 0 & 0 \\
0 & 0 & 0 & 0
\end{array}\right), \quad T_{L}^{3}=\frac{1}{2}\left(\begin{array}{cccc}
1 & 0 & 0 & 0 \\
0 & 0 & 0 & 0 \\
0 & 0 & -1 & 0 \\
0 & 0 & 0 & 0
\end{array}\right), \\
& T_{R}^{1}=\frac{1}{2}\left(\begin{array}{cccc}
0 & 0 & 0 & 0 \\
0 & 0 & 0 & 1 \\
0 & 0 & 0 & 0 \\
0 & 1 & 0 & 0
\end{array}\right), \quad T_{R}^{2}=\frac{1}{2}\left(\begin{array}{cccc}
0 & 0 & 0 & 0 \\
0 & 0 & 0 & -i \\
0 & 0 & 0 & 0 \\
0 & i & 0 & 0
\end{array}\right), \quad T_{R}^{3}=\frac{1}{2}\left(\begin{array}{cccc}
0 & 0 & 0 & 0 \\
0 & 1 & 0 & 0 \\
0 & 0 & 0 & 0 \\
0 & 0 & 0 & -1
\end{array}\right) \text {. }
\end{aligned}
$$

The $T_{L}$ generators satisfy the $S U(2)_{L}$ algebra $\left[T_{L}^{i}, T_{L}^{j}\right]=i \epsilon^{i j k} T_{L}^{k}$, and similarly $\left[T_{R}^{i}, T_{R}^{j}\right]=i \epsilon^{i j k} T_{R}^{k}$, while $\left[T_{L}^{i}, T_{R}^{j}\right]=0$. In the vacuum aligned with $\Omega$ in Eq. (5), this is the natural choice of embedding of the $S O(4)$ symmetries of the Higgs potential. Following the notation in Refs. [50,62], the matrix of the five pNGB fields parametrizing the $S U(4) / S p(4)$ coset is

$$
\pi(x)=\frac{1}{2 \sqrt{2}}\left(\begin{array}{cccc}
\pi^{3}(x) & \pi^{1}(x)-i \pi^{2}(x) & 0 & -i \pi^{4}(x)+\pi^{5}(x) \\
\pi^{1}(x)+i \pi^{2}(x) & -\pi^{3}(x) & i \pi^{4}(x)-\pi^{5}(x) & 0 \\
0 & -i \pi^{4}(x)-\pi^{5}(x) & \pi^{3}(x) & \pi^{1}(x)+i \pi^{2}(x) \\
i \pi^{4}(x)+\pi^{5}(x) & 0 & \pi^{1}(x)-i \pi^{2}(x) & -\pi^{3}(x)
\end{array}\right) .
$$

The real fields $\pi^{1}, \pi^{2}, \pi^{4}$, and $\pi^{5}$ combine into the Higgs doublet, while $\pi^{3}$ is a SM singlet.

The $S U(6) / S O(6)$ coset is relevant to top compositeness. The choice of $n_{f}=3$ Dirac fermions on the 2-index antisymmetric representation of $S p(4)$ matches the number of colours in the $S U(3)_{c}$ gauge group of the Standard Model. The natural subgroup $S U(3)_{L} \times S U(3)_{R} \subset S U(6)$ is generated by

$$
t_{L}^{B}=\frac{1}{2}\left(\begin{array}{c|c}
\lambda^{B} & \mathbb{O}_{3} \\
\hline \mathbb{O}_{3} & \mathbb{O}_{3}
\end{array}\right), \quad t_{R}^{B}=\frac{1}{2}\left(\begin{array}{c|c}
\mathbb{O}_{3} & \mathbb{O}_{3} \\
\hline \mathbb{O}_{3} & -\lambda^{B *}
\end{array}\right),
$$

with $\lambda^{B}$ the eight Hermitian Gell-Mann matrices, normalized according to the relation $\operatorname{Tr} \lambda^{A} \lambda^{B}=2 \delta^{A B}$ (so that $\left.\operatorname{Tr} t_{L}^{A} t_{L}^{B}=\frac{1}{2} \delta^{A B}\right)$.

By defining $t_{c}^{B} \equiv\left(t_{L}^{B}+t_{R}^{B}\right)$, with the choice of $\omega$ in Eq. (5), one can verify that $\omega t_{c}^{B}+t_{c}^{B \mathrm{~T}} \omega=0$, that the structure constants $\left[t_{c}^{A}, t_{c}^{B}\right]=i f^{A B C} t_{c}^{C}$ are those of the $s u(3)_{c}$ algebra, and that $\operatorname{Tr} t_{c}^{A} t_{c}^{B}=\delta^{A B}$ is twice the fundamental. The latter property is due to the fact that we are writing the $S U(3)_{c}$ generators as $6 \times 6$ matrices acting on two-component spinors. We hence identify $t_{c}^{B}$ as the generators of the $S U(3)_{c}$ gauge symmetry of the Standard Model. An additional, independent, unbroken generator of $S U(6)$ is given by

$$
X \equiv\left(\begin{array}{l|r}
\mathbb{1}_{3} & \mathbb{O}_{3} \\
\hline \mathbb{O}_{3} & -\mathbb{1}_{3}
\end{array}\right),
$$

which also commutes with the generators of $S U(3)_{c}$. The generator $Y$ of the hypercharge $U(1)_{Y}$ gauge symmetry of the Standard Model is a linear combination of $X$ and $T_{R}^{3}$ (see also Ref. [36] and references therein). 


\section{Weakly coupling the SM gauge group}

In this Appendix, we perform a technical exercise. We compute the (divergent) contributions to the effective potential due to the gauging of the relevant SM subgroups of the global $S U(4) \times S U(6)$ symmetry and discuss their effects on the potential of the pNGBs. The purpose of this exercise is to show explicitly how by gauging part of the global symmetry one breaks it. We also identify the decomposition of the representations according to the unbroken subgroup.

We adopt the external field method and borrow the regulated Coleman-Weinberg potential $V_{1}$ from Ref. [117], computed by assuming that a hard momentum cutoff $\Lambda$ is applied to the one-loop integrals. With our conventions we write

$V_{1}=\frac{\Lambda^{2}}{32 \pi^{2}} \mathrm{~S} \operatorname{Tr} \mathcal{M}^{2}+\frac{1}{64 \pi^{2}} \mathrm{~S} \operatorname{Tr}\left[\left(\mathcal{M}^{2}\right)^{2} \log \frac{\mathcal{M}^{2}}{\Lambda^{2}}+c_{i}\right]$,

where in the trace STr fermions have negative weight and where $c_{i}$ are scheme-dependent coefficients. The matrix $\mathcal{M}^{2}$ is obtained as follows: consider $\mathcal{L}_{i}$ in Eq. (13), gauge the relevant subgroups, by promoting the derivatives to covariant derivatives, and compute the mass matrices of all the fields, as a function of the (background, external) scalar fields.

When applied to the $S U(4) / S p(4)$ part of the theory (and for $M=0$ ), this procedure involves only loops of gauge bosons and yields a quadratically divergent contribution to the mass of four of the pNGBs-labeled $\pi^{1}, \pi^{2}, \pi^{4}$ and $\pi^{5}$ in Eq. (C3):

$$
\delta_{4} m_{\pi}^{2}=\frac{\Lambda^{2}}{32 \pi^{2}}\left(\frac{9}{2} g_{L}^{2}+\frac{3}{2} g_{R}\right)
$$

where $g_{L}$ is the coupling associated with the $S U(2)_{L}$ group with generators in Eqs. (C1), while $g_{R}$ is the coupling associated with the $U(1)_{R}$ subgroup generated by $T_{R}^{3}$ from Eqs. (C2). The four masses are exactly degenerate, and the mass of $\pi^{3}$ does not receive a correction, as it is associated with a generator that commutes with $S U(2)_{L} \times S U(2)_{R}$, and is hence left unbroken by the weak gauging of the SM gauge group -in practice, the mass of $\pi^{3}$ arises for $M \neq 0$ due to the explicit breaking of the global symmetry of the Lagrangian.

When applied to the 20 pNGBs that describe the $S U(6) / S O(6)$ coset, the loops involve the $S U(3)_{c}$ gauge bosons, with the embedding chosen in this Appendix, and strength $g_{S}$, as well as the $U(1)_{X}$ gauge boson generated by Eq. (C5), with strength $g_{X}$. We find that the mass of $12 \mathrm{pNGBs}$ - transforming as $6_{\mathbb{C}}$ of $S U(3)_{c}$-receive the quadratically divergent contribution

$$
\delta_{12} m_{\pi}^{2}=\frac{\Lambda^{2}}{32 \pi^{2}}\left(10 g_{S}^{2}+24 g_{X}^{2}\right),
$$

and the other eight, which form the adjoint of $S U(3)_{c}$, receive the mass correction

$$
\delta_{8} m_{\pi}^{2}=\frac{\Lambda^{2}}{32 \pi^{2}}\left(9 g_{S}^{2}\right) .
$$

The complex $6_{\mathbb{C}}$ of $S U(3)_{c}$ has nontrivial $U(1)_{X}$ charge, while the eight real components of the adjoint representation of $S U(3)_{c}$ have vanishing $U(1)_{X}$ charge. All 20 pNGBs receive also a degenerate, explicit contribution to their mass, which is controlled by $m$.

\section{APPENDIX D: TOPOLOGICAL CHARGE HISTORY AND MESONIC SPECTRAL OBSERVABLES}

This Appendix reports some technical details and supplementary numerical studies that are not used in the main body of the paper. We saw in Sec. III B that finer lattice spacings were associate with longer autocorrelation times of the topological charge $Q$ (see Fig. 2), with the ensemble with the finest lattice spacing (which we denoted by QB5) showing a particularly long autocorrelation time and a marginal central value of $Q$. To verify that this observation does not affect our main results, we produce a second set of 2400 additional trajectories with the same lattice parameters for QB5, which we call QB5. In Fig. 11 we report the topological history and statistical distribution of QB $\overline{5}$. The
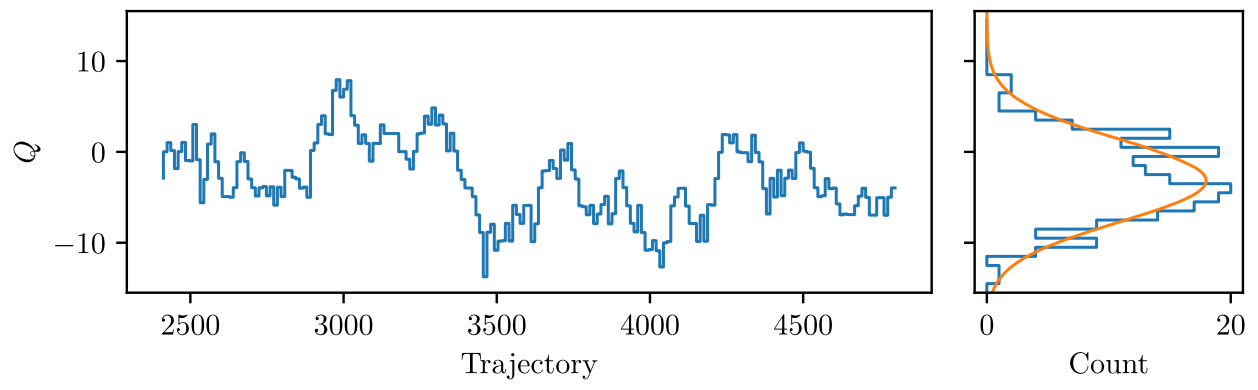

FIG. 11. Topological charge history (left), and histogram (right), for the ensemble QB $\overline{5}$. Fitted parameters are $Q_{0}=-3.17(31)$, $\sigma=4.53(31)$, and $\tau_{\text {exp }}=9.2(1)$. 
TABLE VII. Masses and (renormalized) decay constants, in lattice units, extracted from the measurements QB5FM2 and QB $\overline{5} F M 2$. In parentheses are reported the statistical errors.

\begin{tabular}{lll}
\hline \hline Measurement & \multicolumn{1}{c}{ QB5FM2 } & \multicolumn{1}{c}{ QB $\overline{5} \mathrm{FM} 2$} \\
\hline$a m_{\mathrm{PS}}$ & $0.1850(4)$ & $0.1848(3)$ \\
$a m_{\mathrm{V}}$ & $0.2680(15)$ & $0.2722(17)$ \\
$a m_{\mathrm{AV}}$ & $0.449(7)$ & $0.437(7)$ \\
$a m_{\mathrm{S}}$ & $0.428(6)$ & $0.437(7)$ \\
$a m_{\mathrm{T}}$ & $0.2685(23)$ & $0.2676(25)$ \\
$a m_{\mathrm{AT}}$ & $0.450(9)$ & $0.448(9)$ \\
$a f_{\mathrm{PS}}$ & $0.03740(16)$ & $0.03765(13)$ \\
$a f_{\mathrm{V}}$ & $0.0637(8)$ & $0.0646(9)$ \\
$a f_{\mathrm{AV}}$ & $0.0749(27)$ & $0.0682(21)$ \\
\hline \hline
\end{tabular}

behavior of the topological charge is consistent with ergodicity.

We measure, in the quenched approximation, the masses and (renormalized) decay constants for mesons built of fermions $Q$ transforming in the fundamental representation of $S p(4)$. The results are shown in Table VII. We compare the measurements in QB5FM2 (used in the main analysis in the body of the paper) with the ones from the ensemble QB $\overline{5}$ with the same fermion mass (denoted QB $\overline{5} F M 2$ ). The two sets of measurements are in agreement, within statistical errors, with all nine measurements within $1 \sigma$ and $2 \sigma$ of each other. Notice that systematic uncertainties are not included. In the body of the paper we did not include QB $\overline{5}$ in the analysis.

\section{APPENDIX E: GLOBAL SYMMETRIES AND CLASSIFICATION OF MESONS}

In this Appendix, we review some symmetry properties of the mesons in more general gauge theories of relevance as candidates for the microscopic origin of CHMs. We discuss the cosets that control the long-distance behavior of the theory at low temperatures and describe patterns of symmetry restoration and symmetry enhancement at high temperatures. We keep the discussion as general as possible but occasionally exemplify our observations with the specific case of the $S p(4)$ gauge theory with $N_{f}=2$ and $n_{f}=3$.

Given a Lie group $G$ and its subgroup $H$, a generic element $\mathfrak{g}$ of the associated Lie algebra $\mathcal{G}$ can be decomposed as $\mathfrak{g}=\mathfrak{h}+\mathfrak{f}$, with $\mathfrak{h} \in \mathcal{H}$ an element of the algebra associated with $H$ and $\mathfrak{k} \in \mathcal{K}$ an element of the complement of $\mathcal{H}$ in $\mathcal{G}$. The coset space $G / H$ is said to be symmetric if, for all possible choices of $\mathfrak{h}$ and $\mathfrak{k}$, the following properties are true:

$$
[\mathfrak{h}, \mathfrak{h}] \in \mathcal{H}, \quad[\mathfrak{h}, \mathfrak{f}] \in \mathcal{K}, \quad[\mathfrak{x}, \mathfrak{f}] \in \mathcal{H} .
$$

These properties define in an unambiguous way an unbroken, multiplicative $\mathbb{Z}_{2}$ symmetry, which we can call $G$ parity, which is compatible with the Lie algebra and upon which $\mathfrak{f}$ is assigned $G$ parity -, while $\mathfrak{h}$ is assigned $G$ parity + .

Three classes of cosets are commonly considered in the CHM context (see for instance Table I in Ref. [36]). They all emerge from gauge theories at the microscopic level.

(i) $S U\left(N_{f}\right)_{L} \times S U\left(N_{f}\right)_{R} / S U\left(N_{f}\right)_{V}$ cosets are, for example, realized in $S U(N)$ gauge theories with $N_{f}$ fundamental Dirac fermions.

(ii) $S U\left(2 N_{f}\right) / S p\left(2 N_{f}\right)$ cosets are, for example, realized in $S p(2 N)$ gauge theories with $N_{f}$ fundamental Dirac fermions.

(iii) $S U\left(2 N_{f}\right) / S O\left(2 N_{f}\right)$ cosets are, for example, realized in $S p(2 N)$ gauge theories with $N_{f}$ Dirac fermions transforming in the 2-index antisymmetric representation.

All these cosets are symmetric, and the resulting $G$ parity is a symmetry of the theories. It allows selection rules for scattering and decay processes to be established. We now discuss each of these possibilities in some detail, with emphasis on the properties of the mesons associated with the theories they emerge from.

We begin by reviewing the case of the $S U(3)$ gauge theory with $N_{f}=2$ light flavors. It describes the light mesons in QCD. The associated coset is $S U(2)_{L} \times S U(2)_{R} / S U(2)_{V}$. Much of what one learns from this theory is applicable to the other symmetric cosets listed above, with modifications that will be discussed later. In Table VIII, we report some information about light QCD mesons with $S=C=B=0$, taken from the Particle Data Group [118]. We found Refs. [119-126] and Appendix B in Ref. [127] particularly useful for the discussion that follows.

It is conventional to denote the states of QCD by the quantum numbers $I^{G} J^{P C}$, where $I$ is the isospin [the representation of the unbroken $\left.S U(2)_{V}\right]$ and $J$ the spin. The assignment of $G$ parity for the isotriplets coincides with the traditional $G$ parity: it is related to charge conjugation $\mathcal{C}$ of the neutral component in an isomultiplet, and the isospin $I$ of the isomultiplet, by the relation $G \equiv \mathcal{C}(-1)^{I}$, hence providing a link between the internal symmetry described above and a space-time symmetry. A second subtle link between internal and space-time symmetries involves the notion of spatial parity $P$; the $S U(2)_{L}$ and $S U(2)_{R}$ symmetries act, respectively, on the LH and $\mathrm{RH}$ projections of the spinors, while the unbroken subgroup is the (vectorial) symmetric combination of the two.

We start the discussion from the isotriplets $I=1$. The pions $\pi$ and axial vectors $a_{1}$ are, respectively, the lightest spin- 0 and spin- 1 states and are associated with the broken generators of the global symmetry, so that they have $G=-$; the vector mesons $\rho$ are associated with the unbroken group $S U(2)_{V}$ and have $G=+$. As a result, the $\rho$ decays to two $\pi$ 's, while the $a_{1}$ decays to three $\pi$ 's (or also one $\pi$ and one $\rho$ ). $G$ parity is a useful practical tool: while $\rho$ and $a_{1}$ particles both transform in the adjoint representation of the unbroken $S U(2)_{V}$ (isospin)- the 
TABLE VIII. Light mesons with $S=C=B=0$ in QCD $[118,128]$, their approximate masses, and quantum numbers. Charge conjugation $\mathcal{C}$ refers to the neutral component in multiplets including electrically charge particles, and $G \equiv \mathcal{C}(-1)^{I}$ for the whole multiplet. We show also representative hadronic decay modes. The three-body decay of $\eta$ violates isospin, and hence $G$ parity, and yields $\Gamma / M \sim 10^{-5}$.

\begin{tabular}{lccc}
\hline \hline Particle & $\begin{array}{c}\text { Mass } \\
(\mathrm{MeV})\end{array}$ & $I^{G} J^{P \mathcal{C}}$ & $\begin{array}{c}\text { Hadronic } \\
\text { decay mode(s) }\end{array}$ \\
\hline$f_{0}(500)$ & 500 & $0^{+} 0^{++}$ & $\pi \pi$ \\
$f_{0}(980)$ & 980 & $0^{+} 0^{++}$ & $\pi \pi$ \\
$\eta$ & 548 & $0^{+} 0^{-+}$ & $\pi \pi \pi(\Delta I)$ \\
$\eta^{\prime}$ & 960 & $0^{+} 0^{-+}$ & $\eta \pi \pi$ \\
$\omega$ & 783 & $0^{-} 1^{--}$ & $\pi \pi \pi$ \\
$\phi$ & 1019 & $0^{-} 1^{--}$ & $\bar{K} K$ \\
$f_{1}(1285)$ & 1282 & $0^{+} 1^{++}$ & $4 \pi, \eta \pi \pi$ \\
$f_{1}(1420)$ & 1426 & $0^{+} 1^{++}$ & $\overline{K^{*}} K, \bar{K} K \pi$ \\
$h_{1}$ & 1170 & $0^{-} 1^{+-}$ & $\rho \pi$ \\
$h_{1}^{\prime}$ & $1440[128]$ & $0^{-} 1^{+-}$ & $\overline{K^{*}} K$ \\
$\omega(1420)$ & 1420 & $0^{-} 1^{--}$ & $\rho \pi$ \\
$\phi(1680)$ & 1680 & $0^{-} 1^{--}$ & $\overline{K^{*}} K$ \\
$\pi$ & 135 & $1^{-} 0^{-+}$ & - \\
$a_{0}$ & 980 & $1^{-} 0^{++}$ & $\eta \pi$ \\
$\rho$ & 775 & $1^{+} 1^{--}$ & $\pi \pi$ \\
$a_{1}$ & 1230 & $1^{-} 1^{++}$ & $\rho \pi, \pi \pi \pi$ \\
$b_{1}$ & 1230 & $1^{++} 1^{+-}$ & $\omega \pi$ \\
$\rho(1450)$ & 1465 & $1^{+} 1^{--}$ & $\pi \pi$ \\
\hline \hline & & &
\end{tabular}

unbroken subgroup and the coset have the same dimension-they are distinguished unambiguously by the different assignments of $G$, and hence they decay in different ways.

The global symmetries naturally extend to $U(2)_{L} \times$ $U(2)_{R} / U(2)_{V}$. The additional unbroken vectorial $U(1)_{B}$ is the baryon number, and all mesons have vanishing $U(1)_{B}$ charge. The broken, anomalous, global, axial $U(1)_{A}$ symmetry plays an interesting role in relation to parity $P$. The axial $U(1)_{A}$ partners of the pions $\pi$, named $a_{0}$, have the same $G$ parity but opposite $P$. If the $U(1)_{A}$ were exact, $\pi$ and $a_{0}$ would be degenerate.

In the $J=1, I=1$ sector, the role played by the $U(1)_{A}$ symmetry is more subtle. The $\rho$ and $a_{1}$ mesons are sourced by bilinear operators $\mathrm{V}$ and $\mathrm{AV}$, in which spinor indices are contracted on the $\gamma^{\mu}$ and $\gamma^{\mu} \gamma_{5}$ matrices, respectively. Such operators involve either two LH or two RH chiral spinors: the action of $U(1)_{A}$ leaves them both invariant, as they are two independent singlets of $U(1)_{A}$. But there is an important complication, as massive vectors in four dimensions can equivalently be described by 2 -index antisymmetric tensors (see Appendix B and references therein). Two additional sources of spin- 1 states $\mathrm{T}$ and AT are obtained by contracting the spinor indices on $\sigma^{\mu \nu}$ and $\sigma^{\mu \nu} \gamma_{5}$, respectively. The two operators T and AT couple the $\mathrm{LH}$ and $\mathrm{RH}$ chiral projections of the fermions, in a way that is similar to the $J=0$ isotriplet operators PS and S, that source the $\pi$ and $a_{0}$ particles. They form a doublet of the $U(1)_{A} \sim O(2)$ symmetry.

Because of symmetry breaking, the operator $\mathrm{T}$ (built with $\sigma^{\mu \nu}$ ) has the same quantum numbers $I^{G} J^{P C}=1^{+} 1^{--}$ as the source V (built from $\gamma^{\mu}$ ). The lightest and next-tolightest states in this channel [ $\rho$ and $\rho(1450)$ in Table VIII] can approximately be thought of as resulting from the mixing of two states that have different $S U(2)_{L} \times S U(2)_{R}$ transformation properties and are sourced by different operators $\mathrm{V}$ and $\mathrm{T}$. The $U(1)_{A}$ partner of the combination of $\rho$ and $\rho(1450)$ sourced by T is denoted by $b_{1}$ (see again Table VIII) and sourced by AT.

Because we are also comparing with real-world QCD, we should notice that the isosinglet $I=0$ sector is complicated by the fact that real-world light mesons are better explained by a model in which one includes $2+1$ quarks, including the heavier strange quark $s$. The isosinglet mesons include an additional tower of states, due to mixing with the $(\bar{s} s)$ singlet. In Table VIII, this results in the doubling of states with $I=0$ in respect to the $I=1$ case, as we chose to retain pairs of mesons with identical quantum numbers. The $G$-parity assignment of each isosinglet state is the opposite of that of the corresponding isotriplet with the same $J^{P C}$. Notice in the table that the decay of the $\eta$ to $3 \pi$ violates $G$ parity: it yields a suppressed rate $\Gamma$, with $\Gamma / M \sim 10^{-5}$, and originates from explicit breaking of isospin in real-world QCD, in which, for instance, up and down quarks are not degenerate in mass.

The $I=0$ and $J=0$ lightest states are the $f_{0}(500) / f_{0}(980)$ and their $U(1)_{A}$ partners, the $\eta / \eta^{\prime}$ pair. The $I=0$ and $J=1$ sector contains the pairs $\omega / \phi$, $f_{1}(1285) / f_{1}(1420), h_{1} / h_{1}^{\prime}$, and $\omega(1420) / \phi(1680)$. The four of them play the same roles as, respectively, the $\rho, a_{1}$, $b_{1}$, and $\rho(1450)$ mesons, in the isotriplet case. One linear combination of the two $\omega / \phi$ pairs is sourced by the bilinear operator T, which forms a doublet of $U(1)_{A}$ with the source AT of the $h_{1} / h_{1}^{\prime}$ pair.

Let us see how these considerations have to be modified for enlarged cosets. [Both the $S U\left(2 N_{f}\right) / S p\left(2 N_{f}\right)$ and $S U\left(2 N_{f}\right) / S O\left(2 N_{f}\right)$ contain the $S U\left(N_{f}\right)_{L} \times S U\left(N_{f}\right)_{R} /$ $S U\left(N_{f}\right)_{V}$ subspace, enhanced because of the (pseudo)real nature of the underlying fermion representations.] The unbroken baryon number $U(1)_{B}$ is a subset of the unbroken part of these two cosets rather than commuting with it. Diquark operators hence source mesons that complete the representation of $S U\left(N_{f}\right)_{V}$ into full representations of the unbroken $S p\left(2 N_{f}\right)$ or $S O\left(2 N_{f}\right)$. The unbroken group and the coset have in these cases different dimension, so that representations with different $G$ parity have different dimensionality as well (see Table IV for instance), rendering $G$ parity redundant, at least as a way to distinguish among them. 
We conclude by discussing explicitly the general form of the operators to be used as sources. We start from a twocomponent spinor $\chi$ transforming in the fundamental representation of the global $S U\left(2 N_{f}\right)$ symmetry group. A spin- 0 local operator $J^{0}$ takes the schematic form $\chi^{\mathrm{T}} \tilde{C} \chi$. The product of two fundamental decomposes as

$2 N_{f} \otimes 2 N_{f}=\left[N_{f}\left(2 N_{f}-1\right)\right] \oplus\left[N_{f}\left(2 N_{f}+1\right)\right]$,

into the 2-index antisymmetric and symmetric representations of $S U\left(2 N_{f}\right)$, respectively. Furthermore, depending on whether the unbroken subgroup is $S p\left(2 N_{f}\right)$ or $S O\left(2 N_{f}\right)$, either the antisymmetric or the symmetric combination is reducible and further decomposes into the unbroken subgroup by projecting one element along the elementary symplectic matrix $\Omega$ or the symmetric $\omega$, respectively. Excitations sourced by this operator correspond to the $f_{0}$ of QCD-like theories, while those along the complement correspond to the PS flavored states (the $\pi$ of QCD). The representation that is irreducible, instead, would coincide with the adjoint of the unbroken subgroup, except that it vanishes because of Fermi statistics-unless one considers nonlocal operators.

Along the same lines, the spin-1 local operator $J^{1}$ schematically reads $\chi^{\dagger} \bar{\sigma}^{\mu} \chi$, and the decomposition in $S U\left(2 N_{f}\right)$ takes the form

$$
\overline{2 N_{f}} \otimes 2 N_{f}=1 \oplus\left[4 N_{f}^{2}-1\right] .
$$

After decomposition into the representations of the unbroken subgroup, the adjoint splits into its antisymmetric and symmetric parts, and hence one ultimately finds the same decomposition as in the spin- 0 case. This property descends from the (pseudo)real nature of $\operatorname{Sp}\left(2 N_{f}\right)$ and $S O\left(2 N_{f}\right)$, that do not distinguish the fundamental representation $2 N_{f}$ from its conjugate. It is useful to notice that the four operators $\mathrm{V}, \mathrm{T}, \mathrm{AV}$, and AT states source only one state that is a singlet. This is different from the $S U\left(N_{f}\right)_{L} \times S U\left(N_{f}\right)_{R} /$ $S U\left(N_{f}\right)_{V}$, where each of the four operators sources a singlet of the unbroken group: the three additional $J=1$ states are part of the irreducible representations sourced by $\mathrm{V}, \mathrm{T}$, and AT.

Summarizing for the $S U\left(2 N_{f}\right) / S p\left(2 N_{f}\right)$ and $S U\left(2 N_{f}\right) /$ $S O\left(2 N_{f}\right)$ cases: pseudoscalar PS and axial-vector AV multiplets have the same degeneracies, as do the flavored scalar S-the $U(1)_{A}$ partners of the PS states. The vector states $\mathrm{V}$ belong to a different representation of the unbroken group common also to the antisymmetric tensor T, as well as to its $U(1)_{A}$ partner AT. In the unbroken $S p\left(2 N_{f}\right)$ case, the $\mathrm{V}, \mathrm{T}$ and AT mesons span a complete symmetric representation of $S p\left(2 N_{f}\right)$, with $\mathrm{PS}, \mathrm{S}$, and $\mathrm{AV}$ on the (antisymmetric) complement. The reverse is true in the case of $S O\left(2 N_{f}\right)$ (see again Table IV). In both cases, the singlet sector is simpler: it consists of two spin-0 states forming a $U(1)_{A}$ doublet and of one isolated spin-1 singlet state.

\section{Symmetry restoration and enhancement}

At high temperatures, the fermion condensates melt, leading to restoration of the global symmetries. Both the non-Abelian $S U\left(2 N_{f}\right)$ [or $S U\left(N_{f}\right) \times S U\left(N_{f}\right)$ ] global symmetry, as well as the Abelian $U(1)_{A}$ symmetry are restored [129] (see also [130-132] for progress on $N_{f}=2$ lattice QCD). As a consequence of the former, one might find that the states sourced by $\mathrm{V}$ and $\mathrm{AV}$ operators become degenerate (the $\rho$ and $a_{1}$ in the QCD-like case). Because of the latter, $U(1)_{A}$ multiplets should become degenerate, for example the states sourced by PS and S operators (the $\pi$ and $a_{0}$ mesons in the QCD-like case). See for instance Ref. [50] and references therein.

Recent studies have emerged suggesting that, because the thermal bath reduces the space-time symmetries, the global internal symmetry is further enhanced, with the emergence of a new chiral-spin symmetry that combines with the global symmetries. We refer the reader to Refs. [119-125] for this research field, in which the specific case of the QCD-like, $S U(3)$ theory with $N_{f}=2$ is discussed in great detail, and numerical evidence of the emergence of a $S U(4)$ global symmetry is exposed, in the channels with spin $J>0$. In the following, we limit ourselves to producing a summary of what would be the (testable) expectations in the three main cosets of interest to $\mathrm{CHMs}$, if the corresponding symmetry-restoration and symmetry-enhancement patterns were to be confirmed.

(i) The $S U\left(N_{f}\right)_{L} \times S U\left(N_{f}\right)_{R} / S U\left(N_{f}\right)_{V}$ cosets can emerge, at $T=0$, from theories with complex representations, for example the $S U(N)$ gauge theories with $N_{f}$ fundamental Dirac fermions. These cosets are accompanied by the anomalous $U(1)_{A}=$ $U(1)_{L} \times U(1)_{R} / U(1)_{B}$ Abelian coset. At high temperatures, the global $U\left(N_{f}\right)_{L} \times U\left(N_{f}\right)_{R}$ symmetry is restored and enhanced to $U\left(2 N_{f}\right)=U(1)_{A} \times$ $S U\left(2 N_{f}\right)$. For example, in the $N_{f}=2$ case that approximates QCD, in the $J=1$ sector, four of the $I=1$ states (the two lightest $\rho$, the $a_{1}$, and the $b_{1}$ ) and four of the $I=0$ states (the two $\omega / \phi$, the $f_{1} / f_{1}^{\prime}$, and the $h_{1} / h_{1}^{\prime}$ ) have been measured to become degenerate, which would be compatible with forming a complete 16-dimensional adjoint representation of the $U(1) \times S U(4)$ enhanced global symmetry group [119-126]. In the $J=0$ sector, this symmetry is not manifest: the $\pi$ and $a_{0}$ combine with the $f_{0} / f_{0}^{\prime}$ and the $\eta / \eta^{\prime}$ to form $2 N_{f}^{2}$ degenerate states, the adjoint of the symmetry group $U\left(N_{f}\right)_{L} \times$ $U\left(N_{f}\right)_{R}$. [It would require an additional $2 N_{f}^{2}$ components, two copies of the adjoint of $U\left(N_{f}\right)$, to complete the adjoint of $U\left(2 N_{f}\right)$.]

(ii) The $S U\left(2 N_{f}\right) / S p\left(2 N_{f}\right)$ cosets can emerge, at $T=0$, from theories with pseudoreal representations, for example the $S p(2 N)$ gauge theories with 
$N_{f}$ Dirac fundamental fermions. In addition, the anomalous $U(1)_{A}$ is also spontaneously broken. What would have been the unbroken $U(1)_{B}$ associated with the baryon number in the case of complex representations is now a subgroup of the nonanomalous $\operatorname{Sp}\left(2 N_{f}\right)$. Going to a high temperature, the symmetry is expected to be first restored and then enhanced to $S p\left(4 N_{f}\right)$. For example, in the $N_{f}=2$ case, in the $J=1$ sector of the spectrum, the ten $\mathrm{V}$ and ten $\mathrm{T}$ mesons (which include both the correspondent of the $\rho$ and $\omega / \phi$ of QCD), the five AV mesons (corresponding to the $a_{1}$ ), the ten AT mesons (which include both states corresponding to the $b_{1}$ and $h_{1} / h_{1}^{\prime}$ of QCD) and the singlet vector state (corresponding to the $f_{1} / f_{1}^{\prime}$ ) form a complete, 36-dimensional adjoint representation of $S p(8)$. The $J=0$ sector is not expected to show high- $T$ symmetry enhancement. PS flavored states combine with the singlet to form the antisymmetric representation (in the QCD analogy, they correspond to the $\pi$ and the $f_{0} / f_{0}^{\prime}$ ), and their $U(1)_{A}$ partners (the $a_{0}$ and the $\eta / \eta^{\prime}$ in QCD) combine to form the complex, antisymmetric 2-index representation of $S U\left(2 N_{f}\right)$, which has dimension $2 \times N_{f}\left(2 N_{f}-1\right)$. [It would require finding an adjoint representation of $S U\left(2 N_{f}\right)$ of dimension $4 N_{f}^{2}-1$, to yield a total of $8 N_{f}^{2}-2 N_{f}-1$, which is the 2-index antisymmetric of $S p\left(4 N_{f}\right)$. In the $N_{f}=2$ case, the antisymmetric of $S p(8)$ decomposes as $27=15+6_{\mathbb{C}}$ in terms of $S U(4)$, and the 15 is missing.]

(iii) The $S U\left(2 n_{f}\right) / S O\left(2 n_{f}\right)$ cosets can emerge, at $T=0$, from theories with real representations, for example the $S p(2 N)$ gauge theories with $n_{f}$ Dirac fermions transforming as the 2-index antisymmetric representation. At high temperatures, the restoration of the symmetry is expected to be followed by its enhancement to a global $S O\left(4 n_{f}\right)$. For example, if $n_{f}=3$, in the spin-1 flavored sector the $15 \mathrm{v}, 15 \mathrm{t}$ and 15 at mesons sourced by the operators in Table IV will be degenerate with the $20^{\prime}$ av mesons. In addition, a flavor-singlet vector will also be degenerate and together will yield the 66-dimensional antisymmetric (adjoint) representation of $S O(12)$, with spin $J=1$. In the spin $J=0$ sector, the $20^{\prime}$ ps and $20^{\prime}$ s mesons, together with the $1+1$ flavor singlets form the $21_{\mathbb{C}}$ representation of $S U(6)$. [It would require an additional 35, the adjoint of $S U(6)$, to make the 2-index symmetric traceless 77dimensional representation of $S O(12)$.]

\section{APPENDIX F: BILINEAR OPERATORS AS SOURCES}

We collect in this Appendix technical clarifications about gauge-invariant operators $\mathcal{O}_{M}$, written in terms of the four- component fermions $Q$ and $\Psi$, to be used as sources in the lattice calculations of the spectrum of composite states. We consider the $S p(2 N)$ gauge theory, without specifying $N$. When possible, we also write our expression in a form that applies to $S p\left(2 N_{f}\right)$ and $S O\left(2 n_{f}\right)$ groups with general $N_{f}$ fundamental and $n_{f}$ antisymmetric Dirac fermions. For concreteness, in Appendix F 1 we explicitly identify the irreducible representations of the unbroken global $S p(4)$ group of relevance in the context of CHMs, as well as their $S U(2)_{L} \times S U(2)_{R}$ decompositions.

In the case of $N_{f}$ Dirac spinors transforming in the fundamental representation of the gauge group $S p(2 N)$, the fermion bilinear operators are written as in terms of $N_{f} \times$ $N_{f}$ block matrices, built from the $N_{f}$ Dirac fermions

$$
Q^{i a}=\left(\begin{array}{c}
q^{i a} \\
-\Omega^{a b}\left(\tilde{C} q^{N_{f}+i *}\right)_{b}
\end{array}\right),
$$

and, following the same lines leading to Eqs. (A12), their conjugate Dirac fermion as

$$
\begin{aligned}
Q_{C}^{i a} & \equiv-\gamma_{5} \Omega^{a b} C\left(\bar{Q}^{i \mathrm{~T}}\right)_{b}=-\gamma_{5} \Omega^{a b} C \gamma^{0}\left(Q^{i *}\right)_{b} \\
& =\left(\begin{array}{c}
q^{N_{f}+i a} \\
-\Omega^{a b}\left(\tilde{C} q^{i *}\right)_{b}
\end{array}\right) .
\end{aligned}
$$

Notice a difference, with respect to the definition leading to Eq. (A12), in how we define the conjugate spinor: the factor of $-\gamma_{5} \Omega^{a b}$ is introduced, in order to make the decomposition in LH and RH chiral components of $Q$ and $Q_{C}$ take the same form. We stress that $Q_{C}^{i}$ is physically equivalent to $Q^{i}$, and hence one can identify the $N_{f}$ Dirac fermions with $Q^{i}$.

We write explicitly the form of the general $2 N_{f} \times 2 N_{f}$ matrices built as bilinears in spinors, both in two-component and four-component notation, which read

$$
\begin{aligned}
& J_{0}=\left(\begin{array}{c|c}
\overline{Q_{C}^{i a}} P_{L} Q^{j a} & \overline{Q_{C}^{i a}} P_{L} Q_{C}^{j a} \\
\hline \overline{Q^{i a}} P_{L} Q^{j a} & \overline{Q^{i a}} P_{L} Q_{C}^{j a}
\end{array}\right) \\
& =\Omega_{a b}\left(\begin{array}{c|c}
q^{i a \mathrm{~T}} \tilde{C} q^{j b} & q^{i a \mathrm{~T}} \tilde{C} q^{N_{f}+j b} \\
\hline q^{N_{f}+i a \mathrm{~T}} \tilde{C} q^{j b} & q^{N_{f}+i a \mathrm{~T}} \tilde{C} q^{N_{f}+j b}
\end{array}\right), \\
& J_{1}^{\mu}=\left(\begin{array}{c|c}
\overline{Q^{i a}} \gamma^{\mu} P_{L} Q^{j a} & \overline{Q^{i a}} \gamma^{\mu} P_{L} Q_{C}^{j a} \\
\hline \overline{Q_{C}^{i a}} \gamma^{\mu} P_{L} Q^{j a} & \overline{Q_{C}^{i a}} \gamma^{\mu} P_{L} Q_{C}^{j a}
\end{array}\right) \\
& =\left(\begin{array}{c|c}
q^{i a \dagger} \bar{\sigma}^{\mu} q^{j a} & q^{i a \dagger} \bar{\sigma}^{\mu} q^{N_{f}+j a} \\
\hline q^{N_{f}+i a \dagger} \bar{\sigma}^{\mu} q^{j a} & q^{N_{f}+i a \dagger} \bar{\sigma}^{\mu} q^{N_{f}+j a}
\end{array}\right),
\end{aligned}
$$

and 


$$
\begin{aligned}
J_{1^{\prime}}^{\mu \nu} & =\left(\begin{array}{c|c}
\overline{Q_{C}^{i a}} \sigma^{\mu \nu} P_{L} Q^{j a} & \overline{Q_{C}^{i a}} \sigma^{\mu \nu} P_{L} Q_{C}^{j a} \\
\hline \overline{Q^{i a}} \sigma^{\mu \nu} P_{L} Q^{j a} & \overline{Q^{i a}} \sigma^{\mu \nu} P_{L} Q_{C}^{j a}
\end{array}\right) \\
& =\Omega_{a b}\left(\begin{array}{c|c}
q^{i a \mathrm{~T}} \tilde{C} \sigma_{L L}^{\mu \nu} q^{j b} & q^{i a \mathrm{~T}} \tilde{C} \sigma_{L L}^{\mu \nu} q^{N_{f}+j b} \\
\hline q^{N_{f}+i a \mathrm{~T}} \tilde{C} \sigma_{L L}^{\mu \nu} q^{j b} & q^{N_{f}+i a \mathrm{~T}} \tilde{C} \sigma_{L L}^{\mu \nu} q^{N_{f}+j b}
\end{array}\right) .
\end{aligned}
$$

The symplectic matrix $\Omega_{2 N_{f}}$ is defined as a $2 N_{f} \times 2 N_{f}$ antisymmetric matrix such that $\left(\Omega_{2 N_{f}}\right)^{2}=-\mathbb{1}_{2 N_{f}}$. Because of the contraction with $\Omega_{a b}, J_{0}$ is antisymmetric. This bilinear condenses, and hence the unbroken subgroup is $\operatorname{Sp}\left(2 N_{f}\right)$. The decomposition of $J_{0}, J_{1}^{\mu}$ and $J_{1^{\prime}}^{\mu \nu}$ in their irreducible representations is $2 N_{f} \otimes 2 N_{f}=\left[N_{f}\left(2 N_{f}-1\right)-1\right] \oplus$ $1 \oplus N_{f}\left(2 N_{f}+1\right)$ :

$$
\begin{aligned}
J_{0,1,1^{\prime}}= & {\left[\frac{1}{2}\left(J_{0,1,1^{\prime}}-\left(J_{0,1,1^{\prime}}\right)^{\mathrm{T}}\right)+\frac{\Omega_{2 N_{f}}}{2 N_{f}} \operatorname{Tr}\left\{\Omega_{2 N_{f}} J_{0,1,1^{\prime}}\right\}\right] } \\
& -\frac{\Omega_{2 N_{f}}}{2 N_{f}} \operatorname{Tr}\left\{\Omega_{2 N_{f}} J_{0,1,1^{\prime}}\right\}+\frac{1}{2}\left(J_{0,1,1^{\prime}}+\left(J_{0,1,1^{\prime}}\right)^{\mathrm{T}}\right) \\
\equiv & J_{0,1,1^{\prime}}^{(A)}+J_{0,1,1^{\prime}}^{(1)}+J_{1,1^{\prime}}^{(S)} .
\end{aligned}
$$

We highlighted here the fact that the symmetric part of $J_{0}$ vanishes identically. Notice also that the singlets are antisymmetric. Both the $J_{1}$ and $J_{1^{\prime}}$ decompose into symmetric and antisymmetric parts, the latter expected to be related to heavier states. For the $S U\left(2 N_{f}\right) / S p\left(2 N_{f}\right)$ coset the operators in Table IV are identified as follows:

$$
\mathcal{O}_{\mathrm{PS}}=J_{0}^{(A)}, \quad \mathcal{O}_{\mathrm{V}}=J_{1}^{(S)}, \quad \mathcal{O}_{\mathrm{AV}}=J_{1}^{(A)}, \quad \mathcal{O}_{\mathrm{T}}=J_{1^{\prime}}^{(S)},
$$

with the $\mathcal{O}_{\mathrm{S}}$ and $\mathcal{O}_{\mathrm{AT}}$ operators being the $U(1)_{A}$ conjugates of $\mathcal{O}_{\mathrm{PS}}$ and $\mathcal{O}_{\mathrm{T}}$, respectively.

With matter content including $n_{f}$ Dirac fermions in the antisymmetric representation of $\operatorname{Sp}(2 N)$ (for $N>1$ ) we introduce the analogous $j_{0,1,1^{\prime}}$ operators built from the fermions

$$
\Psi^{i a b}=\left(\begin{array}{c}
\psi^{i a b} \\
-\Omega^{a c} \Omega^{b d}\left(\tilde{C} \psi^{n_{f}+i^{*}}\right)_{c d}
\end{array}\right),
$$

with $i=1, \ldots, n_{f}$, together with their conjugates

$$
\begin{aligned}
\Psi_{C}^{i a b} & \equiv \Omega^{a c} \Omega^{b d} C\left(\overline{\Psi^{i T}}\right)_{c d} \equiv \Omega^{a c} \Omega^{b d} C \gamma^{0}\left(\Psi^{i *}\right)_{c d} \\
& =\left(\begin{array}{c}
\psi^{n_{f}+i a b} \\
-\Omega^{a c} \Omega^{b d}\left(\tilde{C} \psi^{i^{*}}\right)_{c d}
\end{array}\right) .
\end{aligned}
$$

We conventionally align the vacuum with the matrix $\omega_{2 n_{f}}$, the $2 n_{f} \times 2 n_{f}$ symmetric matrix such that $\left(\omega_{2 n_{f}}\right)^{2}=\mathbb{1}_{2 n_{f}}$, generalizing Eq. (5). We decompose $j_{0,1,1^{\prime}}$ in irreducible representations as $2 n_{f} \otimes 2 n_{f}=\left[n_{f}\left(2 n_{f}+1\right)-1\right] \oplus 1 \oplus$ $n_{f}\left(2 n_{f}-1\right)$ :

$$
\begin{aligned}
j_{0,1,1^{\prime}}= & {\left[\frac{1}{2}\left(j_{0,1,1^{\prime}}+\left(j_{0,1,1^{\prime}}\right)^{\mathrm{T}}\right)-\frac{\omega_{2 n_{f}}}{2 n_{f}} \operatorname{Tr}\left\{\omega_{2 n_{f}} j_{0,1,1^{\prime}}\right\}\right] } \\
& +\frac{\omega_{2 n_{f}}}{2 n_{j}} \operatorname{Tr}\left\{\omega_{2 n_{f}} j_{0,1,1^{\prime}}\right\}+\frac{1}{2}\left(j_{0,1,1^{\prime}}-\left(j_{0,1,1^{\prime}}\right)^{\mathrm{T}}\right) \\
\equiv & j_{0,1,1^{\prime}}^{(S)}+j_{0,1,1^{\prime}}^{(1)}+j_{1,1^{\prime}}^{(A)} .
\end{aligned}
$$

We highlighted the fact that the antisymmetric part of $j_{0}$ vanishes. In this case, the singlets are symmetric matrices.

We write these operators in $n_{f} \times n_{f}$ blocks as matrix representations of the global $S U\left(2 n_{f}\right)$ symmetry:

$$
\begin{aligned}
j_{0} & =\left(\begin{array}{l|c}
\overline{\Psi_{C}^{i a b}} P_{L} \Psi^{j a b} & \overline{\Psi_{C}^{i a b}} P_{L} \Psi_{C}^{j a b} \\
\hline \overline{\Psi^{i a b}} P_{L} \Psi^{j a b} & \overline{\Psi^{i a b}} P_{L} \Psi_{C}^{j a b}
\end{array}\right) \\
& =\Omega_{a c} \Omega_{b d}\left(\begin{array}{c|c}
\psi^{i a b \mathrm{~T}} \tilde{C} \psi^{j c d} & \psi^{i a b \mathrm{~T}} \tilde{C} \psi^{n_{f}+j c d} \\
\hline \psi^{n_{f}+i a b \mathrm{~T}} \tilde{C} \psi^{j c d} & \psi^{n_{f}+i a b \mathrm{~T}} \tilde{C} \psi^{n_{f}+j c d}
\end{array}\right),
\end{aligned}
$$

$$
\begin{aligned}
j_{1}^{\mu} & =\left(\begin{array}{c|c}
\overline{\Psi^{i a b}} \gamma^{\mu} P_{L} \Psi^{j a b} & \overline{\Psi^{i a b}} \gamma^{\mu} P_{L} \Psi_{C}^{j a b} \\
\hline \overline{\Psi_{C}^{i a b}} \gamma^{\mu} P_{L} \Psi^{j a b} & \left.\overline{\Psi_{C}^{i a b}} \gamma^{\mu} P_{L} \Psi_{C}^{j a b}\right)
\end{array}\right) \\
& =\left(\begin{array}{c|c}
\psi^{i a b^{\dagger}} \bar{\sigma}^{\mu} \psi^{j a b} & \psi^{i a b \dagger} \bar{\sigma}^{\mu} \psi^{n_{f}+j a b} \\
\hline \psi^{n_{f}+i a b^{\dagger}} \bar{\sigma}^{\mu} \psi^{j a b} & \psi^{n_{f}+i a b^{\dagger}} \bar{\sigma}^{\mu} \psi^{n_{f}+j a b}
\end{array}\right),
\end{aligned}
$$

and

$$
\begin{aligned}
j_{1^{\prime}}^{\mu \nu} & =\left(\begin{array}{c|c}
\overline{\Psi_{C}^{i a b}} \sigma^{\mu \nu} P_{L} \Psi^{j a b} & \overline{\Psi_{C}^{i a b}} \sigma^{\mu \nu} P_{L} \Psi_{C}^{j a b} \\
\hline \overline{\Psi^{i a b}} \sigma^{\mu \nu} P_{L} \Psi^{j a b} & \overline{\Psi^{i a b}} \sigma^{\mu \nu} P_{L} \Psi_{C}^{j a b}
\end{array}\right) \\
& =\Omega_{a c} \Omega_{b d}\left(\begin{array}{c|c}
\psi^{i a b \mathrm{~T}} \tilde{C} \sigma_{L L}^{\mu \nu} \psi^{j c d} & \psi^{i a b \mathrm{~T}} \tilde{C} \sigma_{L L}^{\mu \nu} \psi^{n_{f}+j c d} \\
\hline \psi^{n_{f}+i a b \mathrm{~T}} \tilde{C} \sigma_{L L}^{\mu \nu} \psi^{j c d} & \psi^{n_{f}+i a b \mathrm{~T}} \tilde{C} \sigma_{L L}^{\mu \nu} \psi^{n_{f}+j c d}
\end{array}\right) .
\end{aligned}
$$

In the $S U\left(2 n_{f}\right) / S O\left(2 n_{f}\right)$ case, the operators in Table IV are identified as follows:

$\mathcal{O}_{\mathrm{ps}}=j_{0}^{(S)}, \quad \mathcal{O}_{\mathrm{v}}=j_{1}^{(A)}, \quad \mathcal{O}_{\mathrm{av}}=j_{1}^{(S)}, \quad \mathcal{O}_{\mathrm{t}}=j_{1^{\prime}}^{(A)}$.

Again, $\mathcal{O}_{\mathrm{s}}$ and $\mathcal{O}_{\text {at }}$ operators are the $U(1)_{A}$ conjugates of $\mathcal{O}_{\text {ps }}$ and $\mathcal{O}_{\mathrm{t}}$, respectively.

As explained also in Appendix E, operators $J_{0}$ that source spin- 0 states, and operators $J_{1}$ sourcing spin- 1 states can be classified in terms of the original global $S U\left(2 N_{f}\right)$ (enlarged) symmetry, according to which the former 
transform as $2 N_{f} \otimes 2 N_{f}=\left[N_{f}\left(2 N_{f}-1\right)\right] \oplus\left[N_{f}\left(2 N_{f}+1\right)\right]$, and the latter as $\overline{2 N_{f}} \otimes 2 N_{f}=1 \oplus\left[4 N_{f}^{2}-1\right]$ of $S U\left(2 N_{f}\right)$. Only once the $S U\left(2 N_{f}\right)$ is broken to its $S p\left(2 N_{f}\right)$ group, $J_{0}$ and $J_{1}$ decompose in the same set of irreducible representations of the subgroup. Similarly, operators $J_{1^{\prime}}$ couple LH to RH components of the Dirac fields, and hence while they source particles with the same spin as $J_{1}$, they transform under $S U\left(2 N_{f}\right)$ in the same way as $J_{0}$. Once the symmetry is broken, mixing between the particles sourced by $J_{1}$ and $J_{1^{\prime}}$ will ensue, but in general these are different sources for different $S U\left(2 N_{f}\right)$ particles. The same considerations apply to the $j_{0,1,1^{\prime}}$ operators and their decompositions in irreducible representations of the $S O\left(2 n_{f}\right)$ subgroup.

One can now explicitly decompose the matrices $J_{0,1,1^{\prime}}$ (and $\left.j_{0,1,1^{\prime}}\right)$ into a given basis of the $U\left(N_{f}\right)$ [and $U\left(n_{f}\right)$ ], by making a choice of generators $T^{A}$ (and $t^{B}$ ) of the group. We devote the next subsection to showing the result of this process in the $S U(4) / S p(4)$ case, for some of the interesting operators.

\section{1. $S U(4) / S p(4)$ composite operators}

We focus on the $\operatorname{Sp}(2 N)$ theory with $N_{f}=2$ fundamental fermions, and its $S U(4) / S p(4)$ coset, which is relevant in the CHM context (see also the discussion in [55]).

The pions in Eq. (C3) are sourced by the antisymmetric $\mathcal{O}_{\mathrm{PS}}=J_{0}^{(A)}$ operators. We can add to the Lagrangian density in Eq. (16) the following source term:

$$
\mathcal{L}_{\pi}=\frac{\sqrt{2} f}{2} \operatorname{Tr}\left[J_{0} \Sigma^{*}+\text { H.c. }\right],
$$

where $\Sigma=e^{2 i \pi / f} \Omega$ is the antisymmetric matrix defined in Eq. (12). We can expand for small $\pi / f$ and make use of the antisymmetry in flavor space of $J_{0}$. We make explicit use of the generators as written in Eq. (B.4) of Ref. [50]. Looking at the decomposition of the 5 according to $S O(4) \sim S U(2)_{L} \times S U(2)_{R} \subset S p(4)$, we find that the $S O(4)$-singlet $\pi^{3}$ is sourced by the operator $\mathcal{O}_{\mathrm{PS}, 3} \equiv$ $i \delta \mathcal{L}_{\pi} / \delta \pi^{3}$ that reads

$$
\begin{aligned}
\mathcal{O}_{\mathrm{PS}, 3} & =\frac{i}{2}\left(i J_{0}^{13}-i J_{0}^{31}-i J_{0}^{24}+i J_{0}^{42}+\text { c.c. }\right) \\
& =\left(\overline{Q^{1 a}} \gamma^{5} Q^{1 a}-\overline{Q^{2 a}} \gamma^{5} Q^{2 a}\right)
\end{aligned}
$$

where we made abundant use of Eqs. (A13). The 4 of $S O(4)$ are sourced by the following operators $\mathcal{O}_{\mathrm{PS}, A} \equiv$ $i \delta \mathcal{L}_{\pi} / \delta \pi^{A}$ given by ${ }^{9}$

\footnotetext{
${ }^{9}$ We remind the reader that the sources for $\pi^{1}, \pi^{2}$ and $\pi^{3}$ would be the same sources as for the coset $S U(2)_{L} \times S U(2)_{R} / S U(2)_{V}$. The additional pions are due to the symmetry enhancement in replacing $S U(2 N)$ with $S p(2 N)$ as the gauge group.
}

$$
\begin{aligned}
\mathcal{O}_{\mathrm{PS}, 1} & =\frac{i}{2}\left(+i J_{0}^{14}-i J_{0}^{41}+i J_{0}^{23}-i J_{0}^{32}+\text { c.c. }\right) \\
& =\left(\overline{Q^{1 a}} \gamma^{5} Q^{2 a}+\overline{Q^{2 a}} \gamma^{5} Q^{1 a}\right), \\
\mathcal{O}_{\mathrm{PS}, 2} & =\frac{i}{2}\left(-J_{0}^{14}+J_{0}^{41}+J_{0}^{23}-J_{0}^{32}+\text { c.c. }\right) \\
& =\left(-i \overline{Q^{1 a}} \gamma^{5} Q^{2 a}+i \overline{Q^{2 a}} \gamma^{5} Q^{1 a}\right), \\
\mathcal{O}_{\mathrm{PS}, 4} & =\frac{i}{2}\left(+J_{0}^{12}-J_{0}^{21}-J_{0}^{34}+J_{0}^{43}+\text { c.c. }\right) \\
& =-i\left(\overline{Q^{1 a}} Q_{C}^{2 a}+\overline{Q_{C}^{2 a}} Q^{1 a}\right), \\
\mathcal{O}_{\mathrm{PS}, 5} & =\frac{i}{2}\left(-i J_{0}^{12}+i J_{0}^{21}-i J_{0}^{34}+i J_{0}^{43}+\text { c.c. }\right) \\
& =i\left(-i \overline{Q^{1 a}} Q_{C}^{2 a}+i \overline{Q_{C}^{2 a}} Q^{1 a}\right) .
\end{aligned}
$$

The additional operator given by

$$
\begin{aligned}
\mathcal{O}_{\mathrm{PS}, 0} & \equiv-\frac{1}{2}\left(J_{0}^{13}+J_{0}^{24}-J_{0}^{31}-J_{0}^{42}+\text { c.c }\right) \\
& =\left(\overline{Q^{1 a}} Q^{1 a}+\overline{Q^{2 a}} Q^{2 a}\right)
\end{aligned}
$$

is aligned along $\Omega$, in the internal space. It is the operator that develops a nontrivial VEV. It sources the $S p(4)$ singlet, which has the same role as the $f_{0}(500)$ of QCD (see Table VIII). Notice that these 6 operators put together transform as the antisymmetric representation of the global $S U(4)$, before the decomposition in $S p(4)$ irreducible representations as $6=1+5$.

Six additional operators $\mathcal{O}_{\mathrm{S}, i}$ can be obtained by replacing $\mathbb{1}_{4} \rightarrow i \gamma^{5}$ from the operators $\mathcal{O}_{\mathrm{PS}, i}$ for $i=0, \ldots, 5$. Such operators are related to the former by the (anomalous) global $U(1)_{A} \sim O(2)$ symmetry. [In QCD, the $U(1)_{A}$ partners of the $\pi$ and $f_{0}(500)$ particles are, respectively, the $a_{0}$ and $\eta / \eta^{\prime}$ particles.]

The sources of the spin-1 fields are given in terms of the operators in $J_{1}^{\mu}$. In Sec. II C we introduced the fields $A_{\mu}=\sum_{A=1}^{15} A_{\mu}^{A} T^{i}$ (we drop here the subscript 6), with $T^{A}$ the Hermitian generators of $S U(4)$ normalized as $\operatorname{Tr} T^{A} T^{B}=\frac{1}{2} \delta^{A B}$. As in Ref. [50], it is convenient to label the broken generators with $A=1, \ldots, 5$ and the unbroken ones with $A=6, \ldots, 15$. We hence add to the Lagrangian density the following source term:

$$
\mathcal{L}_{A}=\sqrt{2} \operatorname{Tr}\left[J_{1}^{\mu} A_{\mu}^{\mathrm{T}}\right]+\text { H.c.. }
$$

Starting from the AV sources, we find that $\mathcal{O}_{\mathrm{AV}, A}^{\mu} \equiv \frac{\delta \mathcal{L}_{A}}{\delta A_{\mu}^{A}}$ are given by the following: 


$$
\begin{aligned}
& \mathcal{O}_{\mathrm{AV}, 1}^{\mu}=\frac{1}{2}\left(J_{1}^{12}+J_{1}^{21}+J_{1}^{34}+J_{1}^{43}+\text { c.c. }\right)=\overline{Q^{1 a}} \gamma^{\mu} \gamma^{5} Q^{2 a}+\overline{Q^{2 a}} \gamma^{\mu} \gamma^{5} Q^{1 a}, \\
& \mathcal{O}_{\mathrm{AV}, 2}^{\mu}=\frac{1}{2}\left(-i J_{1}^{12}+i J_{1}^{21}+i J_{1}^{34}-i J_{1}^{43}+\text { c.c. }\right)=-i \overline{Q^{1 a}} \gamma^{\mu} \gamma^{5} Q^{2 a}+i \overline{Q^{2 a}} \gamma^{\mu} \gamma^{5} Q^{1 a}, \\
& \mathcal{O}_{\mathrm{AV}, 3}^{\mu}=\frac{1}{2}\left(J_{1}^{11}-J_{1}^{22}+J_{1}^{33}-J_{1}^{44}+\text { c.c. }\right)=\overline{Q^{1 a}} \gamma^{\mu} \gamma^{5} Q^{1 a}-\overline{Q^{2 a}} \gamma^{\mu} \gamma^{5} Q^{2 a}, \\
& \mathcal{O}_{\mathrm{AV}, 4}^{\mu}=\frac{1}{2}\left(-i J_{1}^{14}+i J_{1}^{23}-i J_{1}^{32}+i J_{1}^{41}+\text { c.c. }\right)=-i \overline{Q^{1}} \gamma^{\mu} Q_{C}^{2}+i \overline{Q_{C}^{2}} \gamma^{\mu} Q^{1}, \\
& \mathcal{O}_{\mathrm{AV}, 5}^{\mu}=\frac{1}{2}\left(J_{1}^{14}-J_{1}^{23}-J_{1}^{32}+J_{1}^{41}+\text { c.c. }\right)=\overline{Q^{1}} \gamma^{\mu} Q_{C}^{2}+\overline{Q_{C}^{2}} \gamma^{\mu} Q^{1},
\end{aligned}
$$

where we used relations such as $\overline{Q^{2 a}} \gamma^{\mu} P_{L} Q_{C}^{1 a}=\left(\overline{Q^{2 a}} \gamma^{\mu} P_{L} Q_{C}^{1 a}\right)^{\mathrm{T}}=-\overline{Q^{1 a}} \gamma^{\mu} P_{R} Q_{C}^{2 a}$, or $\overline{Q_{C}^{1 a}} \gamma^{\mu} P_{L} Q_{C}^{2 a}=\left(\overline{Q_{C}^{1 a}} \gamma^{\mu} P_{L} Q_{C}^{2 a}\right)^{\mathrm{T}}=$ $-\overline{Q^{2 a}} \gamma^{\mu} P_{R} Q^{1 a}$, and $\left(\overline{Q^{2 a}} \gamma^{\mu} P_{L} Q_{C}^{1 a}\right)^{\dagger}=\overline{Q_{C}^{1 a}} \gamma^{\mu} P_{L} Q^{2 a}$. It hence turns out that the $S O(5)$ fundamental 5 decomposes as $4+1$ of $S O(4)$, with the singlet being sourced by $\mathcal{O}_{\mathrm{AV}, 3}^{\mu}$, while the 4 is sourced by $\mathcal{O}_{\mathrm{AV}, 1}^{\mu}, \mathcal{O}_{\mathrm{AV}, 2}^{\mu}, \mathcal{O}_{\mathrm{AV}, 4}^{\mu}$ and $\mathcal{O}_{\mathrm{AV}, 5}^{\mu}$. For completeness, $\mathcal{O}_{\mathrm{AV}, 1}^{\mu}, \mathcal{O}_{\mathrm{AV}, 2}^{\mu}$ and $\mathcal{O}_{\mathrm{AV}, 3}^{\mu}$ would be the generators that are used in the $S U(2)_{L} \times S U(2)_{R} / S U(2)_{V} \operatorname{coset}$.

Similar expressions hold for the ten operators sourcing the $V$ mesons. We adopt the same basis as in Ref. [50], and within these conventions we find the following:

$$
\begin{aligned}
& \mathcal{O}_{\mathrm{V}, 6}^{\mu}=\frac{1}{2}\left(-i J_{1}^{13}-i J_{1}^{24}+i J_{1}^{31}+i J_{1}^{42}+\text { c.c. }\right)=-\frac{i}{2}\left(\overline{Q^{1 a}} \gamma^{\mu} \gamma^{5} Q_{C}^{1 a}+\overline{Q^{2 a}} \gamma^{\mu} \gamma^{5} Q_{C}^{2 a}-\overline{Q_{C}^{1 a}} \gamma^{\mu} \gamma^{5} Q^{1 a}-\overline{Q_{C}^{2 a}} \gamma^{\mu} \gamma^{5} Q^{2 a}\right) \\
& \left.\mathcal{O}_{\mathrm{V}, 7}^{\mu}=\frac{1}{2}\left(-i J_{1}^{14}-i J_{1}^{23}+i J_{1}^{32}+i J_{1}^{41}+\text { c.c. }\right)=-\frac{i}{2} \overline{\left(Q^{1 a}\right.} \gamma^{\mu} \gamma^{5} Q_{C}^{2 a}+\overline{Q^{2 a}} \gamma^{\mu} \gamma^{5} Q_{C}^{1 a}-\overline{Q_{C}^{1 a}} \gamma^{\mu} \gamma^{5} Q^{2 a}-\overline{Q_{C}^{2 a}} \gamma^{\mu} \gamma^{5} Q^{1 a}\right) \\
& \mathcal{O}_{\mathrm{V}, 8}^{\mu}=\frac{1}{2}\left(-i J_{1}^{12}-i J_{1}^{34}+i J_{1}^{21}+i J_{1}^{43}+\text { c.c. }\right)=-i \overline{Q^{1 a}} \gamma^{\mu} Q^{2 a}+i \overline{Q^{2 a}} \gamma^{\mu} Q^{1 a}, \\
& \left.\mathcal{O}_{\mathrm{V}, 9}^{\mu}=\frac{1}{2}\left(-i J_{1}^{13}+i J_{1}^{24}+i J_{1}^{31}-i J_{1}^{42}+\text { c.c. }\right)=-\frac{i}{2} \overline{\left(Q^{1 a}\right.} \gamma^{\mu} \gamma^{5} Q_{C}^{1 a}-\overline{Q^{2 a}} \gamma^{\mu} \gamma^{5} Q_{C}^{2 a}-\overline{Q_{C}^{1 a}} \gamma^{\mu} \gamma^{5} Q^{1 a}+\overline{Q_{C}^{2 a}} \gamma^{\mu} \gamma^{5} Q^{2 a}\right) \\
& \mathcal{O}_{\mathrm{V}, 10}^{\mu}=\frac{\sqrt{2}}{2}\left(J_{1}^{13}+J_{1}^{31}+\text { c.c. }\right)=\frac{\sqrt{2}}{2}\left(\overline{Q^{1 a}} \gamma^{\mu} \gamma^{5} Q_{C}^{1 a}+\overline{Q_{C}^{1 a}} \gamma^{\mu} \gamma^{5} Q^{1 a}\right) \\
& \mathcal{O}_{\mathrm{V}, 11}^{\mu}=\frac{1}{2}\left(J_{1}^{14}+J_{1}^{23}+J_{1}^{32}+J_{1}^{41}+\text { c.c. }\right)=+\frac{1}{2}\left(\overline{Q^{1 a}} \gamma^{\mu} \gamma^{5} Q_{C}^{2 a}+\overline{Q^{2 a}} \gamma^{\mu} \gamma^{5} Q_{C}^{1 a}+\overline{Q_{C}^{1 a}} \gamma^{\mu} \gamma^{5} Q^{2 a}+\overline{Q_{C}^{2 a}} \gamma^{\mu} \gamma^{5} Q^{1 a}\right) \\
& \mathcal{O}_{\mathrm{V}, 12}^{\mu}=\frac{\sqrt{2}}{2}\left(J_{1}^{24}+J_{1}^{42}+\text { c.c. }\right)=\frac{\sqrt{2}}{2}\left(\overline{Q^{2 a}} \gamma^{\mu} \gamma^{5} Q_{C}^{2 a}+\overline{Q_{C}^{2 a}} \gamma^{\mu} \gamma^{5} Q^{2 a}\right) \\
& \mathcal{O}_{\mathrm{V}, 13}^{\mu}=\frac{1}{2}\left(J_{1}^{12}-J_{1}^{34}+J_{1}^{21}-J_{1}^{43}+\text { c.c. }\right)=\overline{Q^{1 a}} \gamma^{\mu} Q^{2 a}+\overline{Q^{2 a}} \gamma^{\mu} Q^{1 a}, \\
& \mathcal{O}_{\mathrm{V}, 14}^{\mu}=\frac{1}{2}\left(J_{1}^{11}-J_{1}^{22}-J_{1}^{33}+J_{1}^{44}+\text { c.c. }\right)=\overline{Q^{1 a}} \gamma^{\mu} Q^{1 a}-\overline{Q^{2 a}} \gamma^{\mu} Q^{2 a}, \\
& \mathcal{O}_{\mathrm{V}, 15}^{\mu}=\frac{1}{2}\left(J_{1}^{11}+J_{1}^{22}-J_{1}^{33}-J_{1}^{44}+\text { c.c. }\right)=\overline{Q^{1 a}} \gamma^{\mu} Q^{1 a}+\overline{Q^{2 a}} \gamma^{\mu} Q^{2 a} .
\end{aligned}
$$

The $S O(5)$ adjoint 10 decomposes as $4+6$ of $S O(4)$ : the fundamental 4 is sourced by $\mathcal{O}_{\mathrm{V}, 7}^{\mu}, \mathcal{O}_{\mathrm{V}, 8}^{\mu}, \mathcal{O}_{\mathrm{V}, 11}^{\mu}$ and $\mathcal{O}_{\mathrm{V}, 13}^{\mu}$, with the adjoint 6 sourced by the other six operators. Again, for completeness, $\mathcal{O}_{\mathrm{V}, 8}^{\mu}, \mathcal{O}_{\mathrm{V}, 13}^{\mu}$ and $\mathcal{O}_{\mathrm{V}, 14}^{\mu}$ would be the unbroken generators in the $S U(2)_{L} \times S U(2)_{R} / S U(2)_{V}$ coset, corresponding to the $\rho$ mesons in $\mathrm{QCD}$, in which case $\mathcal{O}_{\mathrm{V}, 15}^{\mu}$ would be associated with baryon number and would source the $\omega / \phi$ in QCD.

We can introduce an additional vector field $A_{\mu}^{0}$, to complete the adjoint $S U(4)$ to the adjoint of the whole
$U(1) \times S U(4)$, by adding the generator $T^{0}=\frac{1}{2 \sqrt{2}} \mathbb{1}_{4}$. We hence identify the additional operator

$$
\begin{aligned}
\mathcal{O}_{\mathrm{V}, 0}^{\mu} & \equiv \frac{1}{2}\left(J_{1}^{11}+J_{1}^{22}+J_{1}^{33}+J_{1}^{44}+\text { c.c. }\right) \\
& =\overline{Q^{1 a}} \gamma^{\mu} \gamma^{5} Q^{1 a}+\overline{Q^{2 a}} \gamma^{\mu} \gamma^{5} Q^{2 a} .
\end{aligned}
$$

One can recognize this operator to be aligned with the generator of the anomalous $U(1)_{A}$. It sources the equivalent of the $f_{1}(1285)$ of QCD (see Table VIII). 
These 16 operators do not have a $U(1)_{A}$ partner. But a second set of sources of spin-1 states $\mathrm{T}$ is built from $J_{1^{\prime}}^{(S)}$ states, with the $\sigma^{\mu \nu}$ tensor. Their $U(1)_{A}$ partners (corresponding to the more exotic states $b_{1}$ and $h_{1} / h_{1}^{\prime}$ mesons of QCD) are sourced by operators AT involving the tensor $\sigma^{\mu \nu} \gamma_{5}$ (see also [55]). We do not show this explicitly, as it requires performing again the same exercise illustrated in the previous pages. Sourcing even more exotic states such as $\pi_{1}$ may require using derivatives and nonlocal operators [126], which is beyond our current purposes.

\section{APPENDIX G: NUMERICAL RESULTS OF LATTICE MEASUREMENTS}

In this Appendix we present the numerical details and results of lattice measurements for meson masses and decay constants. In Table IX we first list the name of each measurement $\mathrm{QB} i \mathrm{FM} j$ at given values of $\beta$ and $m_{0}$ for fundamental (quenched) fermions and present the time intervals corresponding to the asymptotic region for the cases of PS, V, AV and S mesons along with the values of $\chi^{2} / N_{\text {dof }}$, which reflect the quality of the numerical fits using Eqs. (35) and (36). Similar results for T and AT mesons are shown in Table X. Analogously, in the case of antisymmetric (quenched) fermions, labeled as $\mathrm{QB} i \mathrm{ASM} j$, we presented the results in Table XI for the cases of ps, v, av and s mesons and in Table XII for $\mathrm{t}$ and at mesons.

In Table XIII we present the extracted values of PS masses and decay constants, as well as $\mathrm{S}$ masses. In the table we also present the results of $m_{\mathrm{PS}} L$ and $f_{\mathrm{PS}} L$. Similarly, in Table XIV we show the results of the masses and decay constants of $\mathrm{V}$ and $\mathrm{AV}$ mesons. For presentation

TABLE IX. Technical details about PS, V, AS, and S lattice correlation functions. For each ensemble and each choice of bare mass $a m_{0}$, we show the fitting intervals of the Euclidean time $I_{\text {fit }}=\left[t_{i}, t_{f}\right]$ between the minimum time $t_{i}$ and maximum time $t_{f}$ retained in the single-exponential fit to the measured correlators of mesons made of fundamental Dirac fermions. We carry out a correlated fit via standard $\chi^{2}$ minimization. We report the values of $\chi^{2}$ (normalized by the number of degrees of freedom) at the minima. In the case of $\mathrm{AV}$ and $\mathrm{S}$ states, we leave blank some entries for which the numerical data do not exhibit a plateau in the effective mass plots, because of numerical noise.

\begin{tabular}{|c|c|c|c|c|c|c|c|c|c|}
\hline \multirow[b]{2}{*}{ Measurement } & \multirow[b]{2}{*}{$a m_{0}$} & \multicolumn{2}{|c|}{ PS } & \multicolumn{2}{|c|}{$\mathrm{V}$} & \multicolumn{2}{|c|}{$\mathrm{AV}$} & \multicolumn{2}{|c|}{$\mathrm{S}$} \\
\hline & & $I_{\text {fit }}$ & $\frac{\chi^{2}}{N_{\text {dof }}}$ & $I_{\text {fit }}$ & $\frac{\chi^{2}}{N_{\mathrm{dof}}}$ & $I_{\text {fit }}$ & $\frac{\chi^{2}}{N_{\text {dof }}}$ & $I_{\text {fit }}$ & $\frac{\chi^{2}}{N_{\mathrm{dof}}}$ \\
\hline QB1FM1 & -0.7 & $14-24$ & 1.2 & $13-24$ & 1.1 & $9-13$ & 0.1 & $9-15$ & 0.6 \\
\hline QB1FM2 & -0.73 & $14-24$ & 1.3 & $13-24$ & 1.2 & $9-16$ & 0.2 & $9-16$ & 0.8 \\
\hline QB1FM3 & -0.75 & $14-24$ & 1.3 & $13-24$ & 1.3 & $9-15$ & 0.3 & $9-15$ & 1.1 \\
\hline QB1FM4 & -0.77 & $14-24$ & 1.0 & $12-24$ & 1.8 & $8-11$ & 0.2 & $8-10$ & 0.3 \\
\hline QB1FM5 & -0.78 & $14-24$ & 1.0 & $12-24$ & 1.5 & $8-12$ & 0.3 & $8-10$ & 0.03 \\
\hline QB1FM6 & -0.79 & $14-24$ & 0.7 & $12-24$ & 1.9 & $8-11$ & 0.7 & & \\
\hline QB2FM1 & -0.73 & $15-30$ & 0.9 & $15-30$ & 0.5 & $11-15$ & 1.6 & $10-12$ & 0.2 \\
\hline QB2FM2 & -0.75 & $15-30$ & 0.9 & $15-30$ & 0.6 & $11-15$ & 1.4 & 9-11 & 0.4 \\
\hline QB2FM3 & -0.76 & $15-30$ & 1.0 & $15-30$ & 0.6 & $11-14$ & 0.8 & & \\
\hline QB3FM1 & -0.6 & $22-30$ & 0.6 & 19-30 & 0.7 & $13-26$ & 0.9 & $13-28$ & 0.9 \\
\hline QB3FM2 & -0.65 & $20-30$ & 0.5 & 19-30 & 0.5 & $13-22$ & 0.3 & $13-22$ & 1.5 \\
\hline QB3FM3 & -0.68 & $22-30$ & 0.9 & $21-30$ & 0.7 & $15-22$ & 0.8 & $14-22$ & 0.8 \\
\hline QB3FM4 & -0.7 & $20-30$ & 0.7 & 19-30 & 0.7 & $13-20$ & 0.2 & $10-14$ & 0.6 \\
\hline QB3FM5 & -0.71 & $18-30$ & 1.1 & $20-30$ & 0.6 & $11-15$ & 0.8 & $10-13$ & 0.6 \\
\hline QB3FM6 & -0.72 & $18-30$ & 0.9 & $17-30$ & 0.9 & $11-15$ & 0.3 & & \\
\hline QB3FM7 & -0.73 & $17-30$ & 1.0 & 19-30 & 0.6 & $11-15$ & 1.0 & & \\
\hline QB4FM1 & -0.6 & $22-30$ & 2.2 & $22-30$ & 1.7 & $15-23$ & 0.8 & $16-25$ & 0.6 \\
\hline QB4FM2 & -0.625 & $22-30$ & 1.7 & $22-30$ & 1.6 & $15-23$ & 0.6 & $16-22$ & 0.4 \\
\hline QB4FM3 & -0.64 & $22-30$ & 1.5 & $22-30$ & 1.1 & $15-23$ & 0.7 & $15-22$ & 0.6 \\
\hline QB4FM4 & -0.65 & $22-29$ & 1.1 & $22-30$ & 0.5 & $15-25$ & 0.2 & $15-22$ & 0.6 \\
\hline QB4FM5 & -0.66 & $22-29$ & 1.3 & $20-30$ & 0.6 & $15-24$ & 0.2 & $15-22$ & 0.6 \\
\hline QB4FM6 & -0.67 & $22-28$ & 1.0 & $20-30$ & 0.5 & $15-24$ & 0.3 & $15-22$ & 0.7 \\
\hline QB4FM7 & -0.68 & $19-28$ & 0.8 & $20-29$ & 0.6 & $15-22$ & 0.3 & $13-18$ & 0.2 \\
\hline QB4FM8 & -0.69 & $19-28$ & 0.7 & $20-29$ & 1.0 & & & & \\
\hline QB5FM1 & -0.62 & $23-30$ & 1.0 & $24-30$ & 0.5 & $17-24$ & 0.1 & $15-23$ & 0.8 \\
\hline QB5FM2 & -0.64 & $21-30$ & 0.6 & $21-30$ & 0.6 & $15-24$ & 0.3 & $14-22$ & 1.4 \\
\hline QB5FM3 & -0.646 & $21-30$ & 0.7 & $21-30$ & 0.6 & $17-24$ & 0.7 & & \\
\hline
\end{tabular}


purposes, we find it useful to show also the meson masses in units of the PS decay constant, as well as the ratios of $\hat{f}_{\mathrm{V}} / \hat{f}_{\mathrm{PS}}$, in Table XV. In Table XVI we report the masses of T and AT states, both in units of the lattice spacing $a$ and of the PS decay constant $\hat{f}_{\text {PS }}$. Analogous results for the masses and decay constants of ps, s, v, av, t, and at mesons composed of antisymmetric fermions are presented in Tables XVII-XX.

TABLE X. Technical details about T and AT lattice correlation functions. For each ensemble and each choice of bare mass $a m_{0}$, we show the fitting intervals of the Euclidean time $I_{\mathrm{fit}}=\left[t_{i}, t_{f}\right]$ between the minimum time $t_{i}$ and maximum time $t_{f}$ retained in the singleexponential fit to the measured correlators of mesons made of fundamental Dirac fermions. We carry out a correlated fit via standard $\chi^{2}$ minimization. We report the values of $\chi^{2}$ (normalized by the number of degrees of freedom) at the minima. In the case of the AT state, we leave blank some entries for which the numerical data did not exhibit a plateau in the effective mass plots, because of numerical noise.

\begin{tabular}{lcccc}
\hline \hline & & $T$ & & AT \\
\cline { 2 - 5 } Measurement & $I_{\text {fit }}$ & $\frac{\chi^{2}}{N_{\text {dof }}}$ & $I_{\text {fit }}$ & $\frac{\chi^{2}}{N_{\text {dof }}}$ \\
\hline QB1FM1 & $13-24$ & 0.5 & $9-13$ & 0.7 \\
QB1FM2 & $12-24$ & 0.5 & $9-13$ & 0.2 \\
QB1FM3 & $12-24$ & 0.7 & $8-13$ & 0.1 \\
QB1FM4 & $12-24$ & 1.1 & $8-13$ & 0.1 \\
QB1FM5 & $12-24$ & 0.9 & $8-12$ & 0.6 \\
QB1FM6 & $12-24$ & 1.2 & $8-12$ & 0.4 \\
QB2FM1 & $15-30$ & 1.0 & $11-17$ & 0.8 \\
QB2FM2 & $15-28$ & 1.6 & $11-16$ & 1.4 \\
QB2FM3 & $12-29$ & 0.5 & $9-16$ & 1.0 \\
QB3FM1 & $20-29$ & 0.4 & $13-26$ & 0.9 \\
QB3FM2 & $20-29$ & 1.0 & $13-21$ & 0.4 \\
QB3FM3 & $19-30$ & 0.4 & $13-22$ & 0.6 \\
QB3FM4 & $19-30$ & 1.5 & $13-18$ & 0.9 \\
QB3FM5 & $19-30$ & 0.6 & $11-16$ & 0.8 \\
QB3FM6 & $17-30$ & 1.1 & & 0.8 \\
QB3FM7 & $13-26$ & 1.1 & $15-26$ & 0.3 \\
QB4FM1 & 1.1 & $15-22$ & 0.1 \\
QB4FM2 & $20-30$ & 1.1 & $15-20$ & 0.3 \\
QB4FM3 & $20-30$ & 0.9 & $14-19$ & 0.4 \\
QB4FM4 & $20-28$ & 0.7 & $13-19$ & 0.6 \\
QB4FM5 & $22-30$ & 0.5 & $13-19$ & 0.4 \\
QB4FM6 & $20-30$ & 0.4 & $13-18$ & 0.6 \\
QB4FM7 & $22-30$ & 0.6 & $15-23$ & 0.3 \\
QB4FM8 & $22-30$ & 0.6 & $15-22$ & 0.9 \\
QB5FM1 & $17-30$ & 0.6 & $17-24$ & \\
QB5FM2 & $24-30$ & 0.4 & & \\
QB5FM3 & $20-27$ & & & \\
\hline \hline & $20-30$ & & & \\
\hline
\end{tabular}


TABLE XI. Technical details pertaining the measurements of the correlation functions of operators built with 2-index antisymmetric fermions. For each ensemble and each choice of bare mass $a m_{0}$, we show the fitting intervals of the Euclidean time $I_{\text {fit }}=\left[t_{i}, t_{f}\right]$ between the minimum time $t_{i}$ and maximum time $t_{f}$ retained in the single-exponential fit to the measured correlators of mesons made of antisymmetric Dirac fermions. We carry out a correlated fit via standard $\chi^{2}$ minimization. We report the values of $\chi^{2}$ (normalized by the number of the degrees of freedom) at the minima. In the case of the $\mathrm{v}$, av and s states, we leave blank some entries for which the numerical data do not exhibit a plateau in the effective mass plots, due to numerical noise.

\begin{tabular}{|c|c|c|c|c|c|c|c|c|c|}
\hline \multirow[b]{2}{*}{ Measurement } & \multirow[b]{2}{*}{$m_{0}$} & \multicolumn{2}{|c|}{ ps } & \multicolumn{2}{|c|}{$\mathrm{v}$} & \multicolumn{2}{|c|}{ av } & \multicolumn{2}{|c|}{$\mathrm{s}$} \\
\hline & & $I_{\text {fit }}$ & $\frac{\chi^{2}}{N_{\text {dof }}}$ & $I_{\text {fit }}$ & $\frac{\chi^{2}}{N_{\text {dof }}}$ & $I_{\text {fit }}$ & $\frac{\chi^{2}}{N_{\text {dof }}}$ & $I_{\text {fit }}$ & $\frac{\chi^{2}}{N_{\text {dof }}}$ \\
\hline QB1ASM1 & -1.05 & $14-24$ & 1.2 & $13-24$ & 0.5 & $7-12$ & 0.1 & $10-15$ & 0.5 \\
\hline QB1ASM2 & -1.08 & $12-24$ & 1.9 & $12-24$ & 0.5 & $7-10$ & 0.1 & $7-13$ & 1.8 \\
\hline QB1ASM3 & -1.1 & $12-24$ & 1.1 & $12-24$ & 1.0 & $7-12$ & 1.1 & $8-13$ & 0.7 \\
\hline QB1ASM4 & -1.12 & $12-24$ & 0.8 & $12-20$ & 0.5 & $7-11$ & 0.5 & $7-11$ & 0.2 \\
\hline QB1ASM5 & -1.13 & $11-24$ & 1.6 & $12-19$ & 0.3 & $8-11$ & 0.6 & $7-11$ & 0.4 \\
\hline QB1ASM6 & -1.14 & $13-24$ & 0.7 & $10-20$ & 0.5 & $7-10$ & 0.3 & & \\
\hline QB2ASM1 & -1.05 & $16-30$ & 1.2 & $14-25$ & 1.1 & $9-15$ & 0.7 & $11-17$ & 0.6 \\
\hline QB2ASM2 & -1.08 & $15-30$ & 0.7 & $13-28$ & 0.8 & $9-13$ & 0.1 & $9-14$ & 0.8 \\
\hline QB2ASM3 & -1.09 & $15-30$ & 0.9 & $12-23$ & 0.5 & $10-13$ & 0.5 & $8-12$ & 0.2 \\
\hline QB2ASM4 & -1.1 & $15-30$ & 1.0 & $12-21$ & 0.4 & $8-12$ & 0.2 & $9-12$ & 0.2 \\
\hline QB2ASM5 & -1.11 & $15-30$ & 1.3 & $12-25$ & 0.7 & $9-12$ & 0.2 & $8-12$ & 1.0 \\
\hline QB2ASM6 & -1.12 & $16-30$ & 1.3 & & & & & & \\
\hline QB3ASM1 & -1.03 & $16-30$ & 1.5 & $16-27$ & 0.4 & $10-15$ & 0.2 & $10-18$ & 0.6 \\
\hline QB3ASM2 & -1.04 & $14-30$ & 1.1 & $17-30$ & 1.5 & $10-16$ & 0.1 & $9-16$ & 0.5 \\
\hline QB3ASM3 & -1.05 & $16-30$ & 0.8 & $14-30$ & 0.5 & $9-12$ & 0.2 & $9-15$ & 0.5 \\
\hline QB3ASM4 & -1.06 & $18-30$ & 1.1 & $14-24$ & 0.2 & $9-12$ & 0.3 & & \\
\hline QB4ASM1 & -0.95 & $20-30$ & 0.9 & $20-30$ & 0.8 & $12-21$ & 0.8 & $13-26$ & 0.7 \\
\hline QB4ASM2 & -0.983 & $19-30$ & 1.6 & $20-30$ & 0.9 & $12-19$ & 0.7 & $11-22$ & 0.7 \\
\hline QB4ASM3 & -0.99 & $19-30$ & 1.6 & $17-23$ & 0.8 & $10-18$ & 0.4 & $12-19$ & 0.4 \\
\hline QB4ASM4 & -0.99 & $19-30$ & 1.6 & $17-23$ & 0.8 & $10-18$ & 0.4 & $12-19$ & 0.4 \\
\hline QB4ASM5 & -1.01 & $18-30$ & 0.7 & $17-28$ & 1.1 & $10-16$ & 0.1 & $9-12$ & 0.5 \\
\hline QB4ASM6 & -1.015 & $19-30$ & 0.9 & $16-26$ & 0.5 & $11-15$ & 0.2 & & \\
\hline QB5ASM1 & -0.95 & $20-30$ & 0.6 & $19-30$ & 0.3 & $12-21$ & 0.7 & $12-24$ & 1.0 \\
\hline QB5ASM2 & -0.961 & $19-30$ & 1.7 & $20-30$ & 0.2 & $12-19$ & 0.2 & & \\
\hline
\end{tabular}

TABLE XII. Technical details pertaining the measurements of the correlation functions of operators built with 2-index antisymmetric fermions. For each ensemble and each choice of bare mass $a m_{0}$, we show the fitting intervals of the Euclidean time $I_{\text {fit }}=\left[t_{i}, t_{f}\right]$ between the minimum time $t_{i}$ and maximum time $t_{f}$ retained in the single-exponential fit to the measured correlators of mesons made of antisymmetric Dirac fermions. We carry out a correlated fit via standard $\chi^{2}$ minimization. We report the values of $\chi^{2}$ (normalized by the number of the degrees of freedom) at the minima. In the case of the at state, we leave blank some entries for which the numerical data did not exhibit a plateau in the effective mass plots, due to numerical noise.

\begin{tabular}{|c|c|c|c|c|}
\hline \multirow[b]{2}{*}{ Measurement } & \multicolumn{2}{|c|}{$\mathrm{t}$} & \multicolumn{2}{|c|}{ at } \\
\hline & $I_{\text {fit }}$ & $\frac{\chi^{2}}{N_{\text {dof }}}$ & $I_{\text {fit }}$ & $\frac{\chi^{2}}{N_{\text {dof }}}$ \\
\hline QB1ASM1 & $13-24$ & 1.3 & $9-13$ & 0.7 \\
\hline QB1ASM2 & $11-24$ & 0.8 & $7-11$ & 1.8 \\
\hline QB1ASM3 & $10-20$ & 0.2 & $7-10$ & 0.1 \\
\hline QB1ASM4 & $10-21$ & 1.0 & $8-11$ & 0.1 \\
\hline QB1ASM5 & $11-24$ & 0.4 & $7-9$ & 0.1 \\
\hline QB1ASM6 & $10-15$ & 1.0 & $6-10$ & 0.1 \\
\hline QB2ASM1 & $13-30$ & 0.8 & $9-15$ & 1.0 \\
\hline QB2ASM2 & $14-24$ & 0.7 & $9-14$ & 1.3 \\
\hline QB2ASM3 & $11-20$ & 1.3 & $8-13$ & 0.3 \\
\hline QB2ASM4 & $11-26$ & 1.2 & $7-12$ & 0.2 \\
\hline
\end{tabular}


TABLE XII. (Continued)

\begin{tabular}{|c|c|c|c|c|}
\hline \multirow[b]{2}{*}{ Measurement } & \multicolumn{2}{|c|}{$\mathrm{t}$} & \multicolumn{2}{|c|}{ at } \\
\hline & $I_{\text {fit }}$ & $\frac{\chi^{2}}{N_{\mathrm{dof}}}$ & $I_{\text {fit }}$ & $\frac{\chi^{2}}{N_{\text {dof }}}$ \\
\hline QB2ASM5 & $10-21$ & 0.9 & $11-15$ & 1.4 \\
\hline \multicolumn{5}{|l|}{ QB2ASM6 } \\
\hline QB3ASM1 & 19-30 & 0.8 & $11-15$ & 0.1 \\
\hline QB3ASM2 & $13-30$ & 1.0 & $8-13$ & 0.2 \\
\hline QB3ASM3 & $12-20$ & 0.9 & $9-13$ & 0.2 \\
\hline QB3ASM4 & $12-24$ & 0.2 & $9-12$ & 0.3 \\
\hline QB4ASM1 & $17-30$ & 1.4 & $12-16$ & 1.5 \\
\hline QB4ASM2 & $22-29$ & 0.5 & $11-19$ & 0.3 \\
\hline QB4ASM3 & $17-30$ & 0.7 & $10-16$ & 0.7 \\
\hline QB4ASM4 & $15-30$ & 0.8 & $10-18$ & 1.0 \\
\hline QB4ASM5 & 19-27 & 0.3 & $14-19$ & 0.3 \\
\hline QB4ASM6 & $15-26$ & 0.5 & & \\
\hline QB5ASM1 & $17-30$ & 0.6 & $11-16$ & 0.3 \\
\hline QB5ASM2 & $14-26$ & 0.5 & $10-15$ & 0.1 \\
\hline
\end{tabular}

TABLE XIII. Masses for flavored spin-0 (PS and S) mesons, made of Dirac fermions transforming in the fundamental representations of $S p(4)$, and (renormalized) decay constant of the PS states. All results are obtained in the quenched approximation and presented either in units of the lattice spacing $a$ or volume $L=N_{s} a$.

\begin{tabular}{|c|c|c|c|c|c|}
\hline Measurement & $a m_{\mathrm{PS}}$ & $a f_{\mathrm{PS}}$ & $a m_{\mathrm{S}}$ & $m_{\mathrm{PS}} L$ & $f_{\mathrm{PS}} L$ \\
\hline QB1FM1 & $0.5516(4)$ & $0.08728(26)$ & $0.925(9)$ & $13.239(10)$ & $2.095(6)$ \\
\hline QB1FM2 & $0.4816(5)$ & $0.08206(28)$ & $0.873(11)$ & $11.558(11)$ & $1.969(7)$ \\
\hline QB1FM3 & $0.4309(5)$ & $0.07801(29)$ & $0.840(15)$ & $10.342(12)$ & $1.872(7)$ \\
\hline QB1FM4 & $0.3753(6)$ & $0.0733(3)$ & $0.838(14)$ & $9.008(13)$ & $1.760(8)$ \\
\hline QB1FM5 & $0.3453(6)$ & $0.0709(3)$ & $0.839(19)$ & $8.287(14)$ & $1.702(8)$ \\
\hline QB1FM6 & $0.3125(7)$ & $0.0681(3)$ & & $7.501(16)$ & $1.635(8)$ \\
\hline QB2FM1 & $0.38340(15)$ & $0.06957(9)$ & $0.771(6)$ & $18.403(7)$ & $3.339(4)$ \\
\hline QB2FM2 & $0.32442(17)$ & $0.06482(10)$ & $0.760(7)$ & $15.572(8)$ & $3.112(5)$ \\
\hline QB2FM3 & $0.29148(18)$ & $0.06222(11)$ & & 13.991(9) & $2.986(5)$ \\
\hline QB3FM1 & $0.55219(15)$ & $0.07410(14)$ & $0.7980(29)$ & $26.505(7)$ & $3.557(7)$ \\
\hline QB3FM2 & $0.43873(16)$ & $0.06705(13)$ & $0.711(5)$ & $21.059(7)$ & $3.218(6)$ \\
\hline QB3FM3 & $0.36129(20)$ & $0.06099(12)$ & $0.633(9)$ & $17.342(10)$ & $2.928(6)$ \\
\hline QB3FM4 & $0.30373(21)$ & $0.05644(14)$ & $0.657(4)$ & $14.579(10)$ & $2.728(7)$ \\
\hline QB3FM5 & $0.27138(20)$ & $0.05406(10)$ & $0.640(5)$ & $13.026(10)$ & $2.595(5)$ \\
\hline QB3FM6 & $0.23560(25)$ & $0.05128(13)$ & & $11.309(12)$ & $2.461(6)$ \\
\hline QB3FM7 & $0.19406(25)$ & $0.04841(12)$ & & $9.315(12)$ & $2.324(6)$ \\
\hline QB4FM1 & $0.44146(16)$ & $0.06150(13)$ & $0.649(4)$ & $21.190(8)$ & $2.952(6)$ \\
\hline QB4FM2 & $0.38068(19)$ & $0.05752(13)$ & $0.560(5)$ & $18.272(9)$ & $2.761(6)$ \\
\hline QB4FM3 & $0.34147(20)$ & $0.05471(13)$ & $0.577(5)$ & $16.390(10)$ & $2.626(6)$ \\
\hline QB4FM4 & $0.31413(23)$ & $0.05293(13)$ & $0.540(5)$ & $15.078(11)$ & $2.541(6)$ \\
\hline QB4FM5 & $0.28472(25)$ & $0.05064(13)$ & $0.522(7)$ & $13.666(12)$ & $2.431(6)$ \\
\hline QB4FM6 & $0.25306(27)$ & $0.04816(14)$ & $0.511(10)$ & $12.147(13)$ & $2.312(7)$ \\
\hline QB4FM7 & $0.21806(24)$ & $0.04539(11)$ & $0.523(10)$ & $10.467(11)$ & $2.179(5)$ \\
\hline QB4FM8 & $0.17734(26)$ & $0.04240(12)$ & & $8.512(12)$ & $2.035(6)$ \\
\hline QB5FM1 & $0.2524(3)$ & $0.04282(15)$ & $0.449(4)$ & $12.113(14)$ & $2.055(7)$ \\
\hline QB5FM2 & $0.1850(4)$ & $0.03740(16)$ & $0.428(6)$ & $8.881(17)$ & $1.795(7)$ \\
\hline QB5FM3 & $0.1610(3)$ & $0.03560(14)$ & & $7.727(16)$ & $1.709(7)$ \\
\hline
\end{tabular}


TABLE XIV. Masses and decay constants, computed in the quenched approximation, for flavored spin-1 (V and AV) mesons, made of Dirac fermions transforming in the fundamental representations of $S p(4)$. All results are in units of the lattice spacing $a$. In parentheses we report the statistical uncertainties.

\begin{tabular}{|c|c|c|c|c|}
\hline Measurement & $a m_{\mathrm{V}}$ & $a f_{\mathrm{V}}$ & $a m_{\mathrm{AV}}$ & $a f_{\mathrm{AV}}$ \\
\hline QB1FM1 & $0.6259(8)$ & $0.1372(5)$ & $0.971(9)$ & $0.134(4)$ \\
\hline QB1FM2 & $0.5721(11)$ & $0.1351(7)$ & $0.927(11)$ & $0.138(5)$ \\
\hline QB1FM3 & $0.5356(13)$ & $0.1330(8)$ & $0.902(14)$ & $0.142(6)$ \\
\hline QB1FM4 & 0.4981(18) & $0.1302(10)$ & $0.880(12)$ & $0.148(4)$ \\
\hline QB1FM5 & $0.4793(22)$ & $0.1287(12)$ & $0.865(14)$ & $0.148(5)$ \\
\hline QB1FM6 & $0.4592(27)$ & $0.1262(14)$ & $0.854(17)$ & $0.149(6)$ \\
\hline QB2FM1 & $0.4848(6)$ & $0.1186(4)$ & $0.784(8)$ & $0.115(4)$ \\
\hline QB2FM2 & $0.4474(9)$ & $0.1162(6)$ & $0.745(12)$ & $0.113(5)$ \\
\hline QB2FM3 & $0.4288(12)$ & $0.1148(8)$ & $0.726(15)$ & $0.112(7)$ \\
\hline QB3FM1 & $0.59445(24)$ & $0.10405(21)$ & $0.821(3)$ & $0.0881(15)$ \\
\hline QB3FM2 & $0.4988(3)$ & $0.10087(29)$ & $0.736(5)$ & $0.0930(23)$ \\
\hline QB3FM3 & $0.4394(7)$ & $0.0973(5)$ & $0.664(14)$ & $0.088(8)$ \\
\hline QB3FM4 & $0.4008(8)$ & $0.0959(6)$ & $0.655(11)$ & $0.098(5)$ \\
\hline QB3FM5 & $0.3800(16)$ & $0.0932(11)$ & $0.631(6)$ & $0.0997(20)$ \\
\hline QB3FM6 & $0.3640(15)$ & $0.0944(9)$ & $0.645(10)$ & $0.110(4)$ \\
\hline QB3FM7 & $0.339(4)$ & $0.0874(24)$ & $0.581(11)$ & $0.095(4)$ \\
\hline QB4FM1 & $0.4839(5)$ & $0.08727(28)$ & $0.680(4)$ & $0.0789(17)$ \\
\hline QB4FM2 & $0.4332(4)$ & $0.0851(3)$ & $0.633(5)$ & $0.0809(20)$ \\
\hline QB4FM3 & $0.4023(5)$ & $0.0835(4)$ & $0.605(5)$ & $0.0821(24)$ \\
\hline QB4FM4 & $0.3824(7)$ & $0.0828(5)$ & $0.566(7)$ & $0.0746(28)$ \\
\hline QB4FM5 & $0.3626(7)$ & $0.0821(5)$ & $0.543(8)$ & $0.074(3)$ \\
\hline QB4FM6 & $0.3421(10)$ & $0.0806(6)$ & $0.519(11)$ & $0.072(4)$ \\
\hline QB4FM7 & $0.3222(16)$ & $0.0790(9)$ & $0.492(15)$ & $0.069(6)$ \\
\hline QB4FM8 & $0.303(3)$ & $0.0771(18)$ & & \\
\hline QB5FM1 & $0.3112(7)$ & $0.0665(5)$ & $0.480(6)$ & $0.0703(27)$ \\
\hline QB5FM2 & $0.2680(15)$ & $0.0637(8)$ & $0.449(7)$ & $0.0749(27)$ \\
\hline QB5FM3 & $0.2589(23)$ & $0.0637(11)$ & $0.406(16)$ & $0.063(6)$ \\
\hline
\end{tabular}

TABLE XV. Some useful ratios of (quenched) masses and decay constants of mesons made of Dirac fermions transforming in the fundamental representation. In parentheses we report the statistical uncertainties.

\begin{tabular}{|c|c|c|c|c|c|c|}
\hline Measurement & $\hat{m}_{\mathrm{V}} / \hat{m}_{\mathrm{PS}}$ & $\hat{m}_{\mathrm{PS}} / \hat{f}_{\mathrm{PS}}$ & $\hat{m}_{\mathrm{V}} / \hat{f}_{\mathrm{PS}}$ & $\hat{m}_{\mathrm{AV}} / \hat{f}_{\mathrm{PS}}$ & $\hat{m}_{\mathrm{S}} / \hat{f}_{\mathrm{PS}}$ & $\hat{f}_{\mathrm{V}} / \hat{f}_{\mathrm{PS}}$ \\
\hline QB1FM1 & $1.1346(13)$ & $6.320(17)$ & $7.171(23)$ & $11.13(11)$ & $10.60(10)$ & $1.572(7)$ \\
\hline QB1FM2 & $1.1880(20)$ & $5.869(17)$ & $6.972(27)$ & $11.30(14)$ & $10.64(13)$ & $1.646(9)$ \\
\hline QB1FM3 & $1.2429(29)$ & $5.523(18)$ & $6.86(3)$ & $11.56(18)$ & $10.77(18)$ & $1.704(11)$ \\
\hline QB1FM4 & $1.327(5)$ & $5.120(19)$ & $6.79(4)$ & $12.01(17)$ & $11.43(19)$ & $1.776(15)$ \\
\hline QB1FM5 & $1.388(6)$ & $4.869(20)$ & $6.76(4)$ & $12.20(20)$ & $11.83(26)$ & $1.815(17)$ \\
\hline QB1FM6 & $1.469(8)$ & $4.587(21)$ & $6.74(5)$ & $12.54(20)$ & & $1.852(21)$ \\
\hline QB2FM1 & $1.2646(14)$ & $5.511(7)$ & $6.969(12)$ & $11.26(12)$ & $11.08(10)$ & $1.705(6)$ \\
\hline QB2FM2 & $1.3791(27)$ & $5.005(7)$ & $6.902(17)$ & $11.50(18)$ & $11.73(11)$ & $1.793(9)$ \\
\hline QB2FM3 & $1.471(4)$ & $4.685(7)$ & $6.891(22)$ & $11.67(25)$ & & $1.845(12)$ \\
\hline QB3FM1 & $1.0765(4)$ & $7.452(14)$ & $8.022(16)$ & $11.08(5)$ & $10.76(5)$ & $1.404(3)$ \\
\hline QB3FM2 & $1.1370(7)$ & $6.544(12)$ & $7.440(15)$ & $10.97(8)$ & 10.61(8) & $1.505(4)$ \\
\hline QB3FM3 & $1.2161(18)$ & $5.924(10)$ & $7.204(18)$ & $11.70(11)$ & $10.39(15)$ & $1.596(9)$ \\
\hline QB3FM4 & $1.3196(27)$ & $5.343(12)$ & $7.051(22)$ & $11.52(19)$ & $11.56(8)$ & $1.687(10)$ \\
\hline QB3FM5 & $1.400(6)$ & $5.020(8)$ & $7.03(3)$ & $11.66(11)$ & $11.84(10)$ & $1.725(20)$ \\
\hline QB3FM6 & $1.545(7)$ & $4.595(11)$ & $7.10(3)$ & $12.41(15)$ & & $1.841(21)$ \\
\hline QB3FM7 & $1.745(21)$ & $4.009(9)$ & $7.00(8)$ & $12.01(24)$ & & $1.81(5)$ \\
\hline QB4FM1 & $1.0961(6)$ & $7.179(14)$ & $7.868(17)$ & $11.06(7)$ & $10.55(7)$ & $1.419(4)$ \\
\hline QB4FM2 & 1.1381(10) & $6.618(14)$ & $7.532(18)$ & $11.01(8)$ & $10.42(10)$ & $1.480(6)$ \\
\hline
\end{tabular}


TABLE XV. (Continued)

\begin{tabular}{|c|c|c|c|c|c|c|}
\hline Measurement & $\hat{m}_{\mathrm{V}} / \hat{m}_{\mathrm{PS}}$ & $\hat{m}_{\mathrm{PS}} / \hat{f}_{\mathrm{PS}}$ & $\hat{m}_{\mathrm{V}} / \hat{f}_{\mathrm{PS}}$ & $\hat{m}_{\mathrm{AV}} / \hat{f}_{\mathrm{PS}}$ & $\hat{m}_{\mathrm{S}} / \hat{f}_{\mathrm{PS}}$ & $\hat{f}_{\mathrm{V}} / \hat{f}_{\mathrm{PS}}$ \\
\hline QB4FM3 & $1.1782(14)$ & $6.242(14)$ & $7.354(20)$ & $11.06(10)$ & $10.54(11)$ & $1.527(7)$ \\
\hline QB4FM4 & $1.2175(20)$ & $5.945(12)$ & $7.226(21)$ & $10.69(13)$ & $10.19(10)$ & $1.565(10)$ \\
\hline QB4FM5 & $1.2735(25)$ & $5.634(12)$ & $7.160(24)$ & $10.71(16)$ & $10.31(13)$ & $1.621((10)$ \\
\hline QB4FM6 & $1.352(4)$ & $5.255(12)$ & 7.104(29) & $10.77(22)$ & $10.60(20)$ & $1.674(13)$ \\
\hline QB4FM7 & $1.478(7)$ & $4.804(11)$ & $7.10(4)$ & $10.8(3)$ & $11.53(21)$ & $1.741(20)$ \\
\hline QB4FM8 & $1.708(19)$ & $4.183(11)$ & 7.14(9) & & & $1.82(4)$ \\
\hline QB5FM1 & $1.2332(27)$ & $5.894(18)$ & $7.268(27)$ & $11.21(15)$ & $10.49(9)$ & $1.552(10)$ \\
\hline QB5FM2 & $1.449(8)$ & $4.948(19)$ & $7.17(4)$ & $12.02(20)$ & $11.44(18)$ & $1.703(19)$ \\
\hline QB5FM3 & $1.608(14)$ & $4.522(18)$ & $7.27(7)$ & $11.4(4)$ & & $1.789(29)$ \\
\hline
\end{tabular}

TABLE XVI. Masses of $T$ and $A T$ states, in units of $a$ and $f_{\mathrm{PS}}$, for each of the ensembles and bare masses $m_{0}$. The Dirac fermions are in the fundamental representation. In parentheses we report the statistical uncertainties.

\begin{tabular}{|c|c|c|c|c|}
\hline Measurement & $a m_{\mathrm{T}}$ & $\hat{m}_{\mathrm{T}} / \hat{f}_{\mathrm{PS}}$ & $a m_{\mathrm{AT}}$ & $\hat{m}_{\mathrm{AT}} / \hat{f}_{\mathrm{PS}}$ \\
\hline QB1FM1 & $0.6257(10)$ & $7.169(23)$ & $0.963(10)$ & $11.03(11)$ \\
\hline QB1FM2 & $0.5719(14)$ & $6.969(28)$ & $0.920(12)$ & $11.21(15)$ \\
\hline QB1FM3 & $0.5350(18)$ & $6.86(3)$ & $0.899(10)$ & $11.52(13)$ \\
\hline QB1FM4 & $0.4978(25)$ & $6.79(4)$ & $0.863(14)$ & $11.77(20)$ \\
\hline QB1FM5 & $0.477(3)$ & $6.73(5)$ & $0.858(15)$ & $12.09(23)$ \\
\hline QB1FM6 & $0.459(4)$ & $6.74(7)$ & $0.834(19)$ & $12.2(3)$ \\
\hline QB2FM1 & $0.4838(9)$ & $6.954(15)$ & $0.775(10)$ & $11.13(15)$ \\
\hline QB2FM2 & $0.4465(15)$ & $6.887(24)$ & $0.741(15)$ & $11.42(24)$ \\
\hline QB2FM3 & $0.4307(15)$ & $6.922(27)$ & $0.754(8)$ & $12.12(13)$ \\
\hline QB3FM1 & $0.5944(3)$ & $8.021(16)$ & $0.8201(4)$ & $11.07(6)$ \\
\hline QB3FM2 & $0.4986(5)$ & $7.437(17)$ & $0.735(6)$ & $10.96(9)$ \\
\hline QB3FM3 & $0.4397(9)$ & $7.209(12)$ & $0.673(7)$ & $11.04(12)$ \\
\hline QB3FM4 & $0.3997(14)$ & $7.03(3)$ & $0.661(14)$ & $11.63(26)$ \\
\hline QB3FM5 & $0.3806(23)$ & 7.04(4) & $0.596(20)$ & $11.0(4)$ \\
\hline QB3FM6 & $0.3649(26)$ & $7.12(5)$ & $0.672(12)$ & $13.11(24)$ \\
\hline QB3FM7 & $0.3532(29)$ & $7.30(6)$ & & \\
\hline QB4FM1 & $0.4844(4)$ & 7.877(17) & $0.675(4)$ & $10.97(8)$ \\
\hline QB4FM2 & $0.4336(5)$ & $7.538(20)$ & $0.626(6)$ & $10.88(10)$ \\
\hline QB4FM3 & $0.4023(7)$ & $7.354(23)$ & $0.595(7)$ & $10.87(13)$ \\
\hline QB4FM4 & $0.3824(11)$ & $7.226(26)$ & $0.581(7)$ & $10.98(13)$ \\
\hline QB4FM5 & $0.3617(15)$ & $7.14(3)$ & $0.657(6)$ & $11.19(11)$ \\
\hline QB4FM6 & $0.3405(23)$ & $7.07(5)$ & $0.547(7)$ & $11.36(15)$ \\
\hline QB4FM7 & $0.318(4)$ & 7.01(9) & $0.525(9)$ & $11.59(20)$ \\
\hline QB4FM8 & $0.303(4)$ & 7.14(9) & & \\
\hline QB5FM1 & $0.3111(14)$ & $7.27(4)$ & $0.488(5)$ & $11.39(13)$ \\
\hline QB5FM2 & $0.2685(23)$ & $7.18(6)$ & $0.450(9)$ & $12.03(25)$ \\
\hline QB5FM3 & $0.252(3)$ & 7.08(9) & $0.391(19)$ & $11.0(5)$ \\
\hline
\end{tabular}


TABLE XVII. Masses for flavored spin-0 (ps and s) mesons, made of Dirac fermions transforming in the 2-index antisymmetric representations of $S p(4)$, and decay constant of the PS states. All results are obtained in the quenched approximation and presented either in units of the lattice spacing $a$, or volume $L$. In parentheses we report the statistical uncertainties.

\begin{tabular}{|c|c|c|c|c|c|}
\hline Measurement & $a m_{\mathrm{ps}}$ & $a f_{\mathrm{ps}}$ & $a m_{\mathrm{s}}$ & $m_{\mathrm{ps}} L$ & $f_{\mathrm{ps}} L$ \\
\hline QB1ASM1 & $0.6254(4)$ & $0.1249(4)$ & $1.045(28)$ & $15.009(9)$ & 2.997(9) \\
\hline QB1ASM2 & $0.5413(4)$ & $0.1166(3)$ & $1.036(10)$ & 12.991(10) & $2.798(8)$ \\
\hline QB1ASM3 & $0.4789(4)$ & $0.1107(4)$ & $0.970(20)$ & $11.495(9)$ & $2.657(9)$ \\
\hline QB1ASM4 & $0.4087(5)$ & $0.1036(3)$ & $0.953(16)$ & $9.809(11)$ & $2.487(8)$ \\
\hline QB1ASM5 & $0.3693(5)$ & $0.0998(4)$ & $0.930(25)$ & $8.863(12)$ & $2.396(8)$ \\
\hline QB1ASM6 & $0.3260(5)$ & $0.0958(5)$ & & $7.823(13)$ & $2.300(12)$ \\
\hline QB2ASM1 & $0.50776(12)$ & $0.10646(13)$ & $0.894(11)$ & $24.372(6)$ & $5.110(6)$ \\
\hline QB2ASM2 & $0.40809(14)$ & $0.09668(18)$ & $0.851(8)$ & $19.588(7)$ & $4.641(6)$ \\
\hline QB2ASM3 & $0.37047(16)$ & $0.09300(14)$ & $0.860(7)$ & $17.782(8)$ & $4.464(7)$ \\
\hline QB2ASM4 & $0.32896(16)$ & $0.08898(14)$ & $0.824(14)$ & $15.790(8)$ & $4.271(7)$ \\
\hline QB2ASM5 & $0.28241(19)$ & $0.08494(16)$ & $0.842(14)$ & $13.556(9)$ & $4.077(8)$ \\
\hline QB2ASM6 & $0.22727(22)$ & $0.08108(22)$ & & $10.909(10)$ & $3.892(10)$ \\
\hline QB3ASM1 & $0.35682(16)$ & $0.08149(13)$ & $0.726(6)$ & $17.127(8)$ & $3.912(6)$ \\
\hline QB3ASM2 & $0.31698(16)$ & $0.07781(13)$ & $0.704(5)$ & $15.215(8)$ & $3.735(6)$ \\
\hline QB3ASM3 & $0.27265(21)$ & $0.07361(18)$ & $0.698(7)$ & $13.087(10)$ & $3.533(8)$ \\
\hline QB3ASM4 & $0.22041(27)$ & $0.06926(19)$ & & $10.580(13)$ & $3.325(10)$ \\
\hline QB4ASM1 & $0.44487(15)$ & $0.08239(15)$ & $0.692(4)$ & $21.354(7)$ & $3.945(7)$ \\
\hline QB4ASM2 & $0.33323(16)$ & $0.08239(15)$ & $0.623(4)$ & $15.995(8)$ & $3.444(6)$ \\
\hline QB4ASM3 & $0.30578(19)$ & $0.06921(15)$ & $0.611(7)$ & $14.678(9)$ & $3.322(7)$ \\
\hline QB4ASM4 & $0.26323(18)$ & $0.06536(15)$ & $0.579(9)$ & $12.635(9)$ & $3.137(7)$ \\
\hline QB4ASM5 & $0.21375(20)$ & $0.06080(17)$ & $0.604(7)$ & $10.260(10)$ & $2.918(8)$ \\
\hline QB4ASM6 & $0.18506(25)$ & $0.05838(17)$ & & $8.883(12)$ & $2.802(8)$ \\
\hline QB4ASM1 & $0.22454(27)$ & $0.05392(13)$ & $0.484(5)$ & $10.778(13)$ & $2.588(6)$ \\
\hline QB4ASM2 & $0.1666(3)$ & $0.04851(15)$ & & 7.999(15) & $2.329(7)$ \\
\hline
\end{tabular}

TABLE XVIII. Masses and decay constants, computed in the quenched approximation, for flavored spin-1 (v and av) mesons, made of Dirac fermions transforming in the antisymmetric representations of $S p(4)$. All results are in units of the lattice spacing $a$. In parentheses we report the statistical uncertainties.

\begin{tabular}{lllll}
\hline \hline Measurement & \multicolumn{1}{c}{$a m_{\mathrm{v}}$} & $a f_{\mathrm{v}}$ & $a m_{\mathrm{av}}$ & $a f_{\mathrm{av}}$ \\
\hline QB1ASM1 & $0.7457(11)$ & $0.1970(11)$ & $1.216(10)$ & $0.196(5)$ \\
QB1ASM2 & $0.6836(12)$ & $0.1924(11)$ & $1.146(13)$ & $0.192(6)$ \\
QB1ASM3 & $0.6393(19)$ & $0.1862(17)$ & $1.082(18)$ & $0.183(7)$ \\
QB1ASM4 & $0.595(3)$ & $0.1842(25)$ & $1.083(22)$ & $0.205(11)$ \\
QB1ASM5 & $0.571(4)$ & $0.179(3)$ & $0.94(5)$ & $0.148(18)$ \\
QB1ASM6 & $0.542(4)$ & $0.1759(21)$ & $1.01(4)$ & $0.190(16)$ \\
QB2ASM1 & $0.6378(7)$ & $0.1726(7)$ & $1.020(9)$ & $0.159(5)$ \\
QB2ASM2 & $0.5679(8)$ & $0.1645(7)$ & $0.942(14)$ & $0.154(7)$ \\
QB2ASM3 & $0.5466(10)$ & $0.1646(7)$ & $0.96(3)$ & $0.174(22)$ \\
QB2ASM4 & $0.5222(12)$ & $0.1615(8)$ & $0.937(13)$ & $0.172(6)$ \\
QB2ASM5 & $0.4921(22)$ & & $0.871(28)$ & $0.152(13)$ \\
QB2ASM6 & & & \\
\hline
\end{tabular}


TABLE XVIII. (Continued)

\begin{tabular}{lllll}
\hline \hline Measurement & \multicolumn{1}{c}{$a m_{\mathrm{v}}$} & \multicolumn{1}{c}{$a f_{\mathrm{v}}$} & \multicolumn{1}{c}{$a m_{\mathrm{av}}$} \\
\hline QB3ASM1 & $0.4933(11)$ & $0.1346(9)$ & $0.800(10)$ & $0.130(5)$ \\
QB3ASM2 & $0.4637(19)$ & $0.1290(16)$ & $0.785(12)$ & $0.135(6)$ \\
QB3ASM3 & $0.4461(18)$ & $0.1325(12)$ & $0.778(11)$ & $0.142(5)$ \\
QB3ASM4 & $0.417(3)$ & $0.1276(20)$ & $0.752(14)$ & $0.141(6)$ \\
QB4ASM1 & $0.5287(6)$ & $0.1221(6)$ & $0.770(6)$ & $0.1060(29)$ \\
QB4ASM2 & $0.4425(12)$ & $0.1122(11)$ & $0.691(10)$ & $0.108(4)$ \\
QB4ASM3 & $0.4249(12)$ & $0.1115(9)$ & $0.700(5)$ & $0.1222(19)$ \\
QB4ASM4 & $0.3960(20)$ & $0.1076(15)$ & $0.660(10)$ & $0.115(4)$ \\
QB4ASM5 & $0.3697(25)$ & $0.1060(16)$ & $0.629(10)$ & $0.115(4)$ \\
QB4ASM6 & $0.348(4)$ & $0.0995(19)$ & $0.662(22)$ & $0.134(11)$ \\
QB5ASM1 & $0.3327(14)$ & $0.0865(9)$ & $0.558(7)$ & $0.1006(28)$ \\
QB5ASM2 & $0.301(5)$ & $0.0840(26)$ & $0.499(9)$ \\
\hline \hline
\end{tabular}

TABLE XIX. Some useful ratios of (quenched) masses and decay constants of mesons made of Dirac fermions transforming in the antisymmetric representation. In parentheses we report the statistical uncertainties.

\begin{tabular}{|c|c|c|c|c|c|c|}
\hline Measurement & $\hat{m}_{\mathrm{v}} / \hat{m}_{\mathrm{ps}}$ & $\hat{m}_{\mathrm{ps}} / \hat{f}_{\mathrm{ps}}$ & $\hat{m}_{\mathrm{v}} / \hat{f}_{\mathrm{ps}}$ & $\hat{m}_{\mathrm{av}} / \hat{f}_{\mathrm{ps}}$ & $\hat{m}_{\mathrm{s}} / \hat{f}_{\mathrm{ps}}$ & $\hat{f}_{\mathrm{v}} / \hat{f}_{\mathrm{pv}}$ \\
\hline QB1ASM1 & $1.1924(17)$ & $5.009(14)$ & $5.972(21)$ & $9.74(8)$ & $8.37(22)$ & $1.578(10)$ \\
\hline QB1ASM2 & $1.2629(23)$ & $4.643(12)$ & $5.864(19)$ & $9.83(12)$ & $8.89(9)$ & $1.651(10)$ \\
\hline QB1ASM3 & $1.335(4)$ & $4.326(14)$ & $5.774(25)$ & $9.77(17)$ & $8.76(18)$ & $1.682(14)$ \\
\hline QB1ASM4 & $1.457(8)$ & $3.944(11)$ & $5.75(3)$ & $10.45(21)$ & $9.20(15)$ & $1.778(24)$ \\
\hline QB1ASM5 & $1.545(11)$ & $3.699(12)$ & $5.72(5)$ & $9.4(5)$ & $9.31(25)$ & $1.79(3)$ \\
\hline QB1ASM6 & $1.654(15)$ & $3.401(16)$ & $5.65(5)$ & $10.5(4)$ & & $1.835(23)$ \\
\hline QB2ASM1 & $1.2561(13)$ & $4.770(5)$ & $5.991(9)$ & $9.57(9)$ & $8.40(11)$ & $1.621(6)$ \\
\hline QB2ASM2 & $1.3916(21)$ & $4.221(5)$ & $5.874(12)$ & $9.74(14)$ & $8.81(8)$ & $1.701(8)$ \\
\hline QB2ASM3 & $1.4754(27)$ & $3.983(5)$ & $5.877(14)$ & $10.3(4)$ & $9.24(8)$ & $1.770(8)$ \\
\hline QB2ASM4 & $1.587(4)$ & $3.697(5)$ & $5.869(16)$ & $10.53(15)$ & $9.27(15)$ & $1.815(10)$ \\
\hline QB2ASM5 & $1.742(8)$ & $3.325(5)$ & $5.793(25)$ & $10.3(3)$ & $9.91(17)$ & $1.823(16)$ \\
\hline QB2ASM6 & & $2.803(7)$ & & & & \\
\hline QB3ASM1 & $1.382(3)$ & $4.379(6)$ & $6.053(15)$ & $9.81(13)$ & $8.91(8)$ & $1.652(10)$ \\
\hline QB3ASM2 & $1.463(6)$ & $4.073(7)$ & $5.959(27)$ & $10.09(15)$ & $9.05(7)$ & $1.657(21)$ \\
\hline QB3ASM3 & $1.636(7)$ & $3.704(8)$ & $6.061(28)$ & $10.56(15)$ & $9.48(10)$ & $1.801(16)$ \\
\hline QB3ASM4 & 1.891(15) & $3.182(8)$ & $6.02(5)$ & $10.86(21)$ & & $1.843(29)$ \\
\hline QB4ASM1 & $1.1884(13)$ & $5.399(9)$ & $6.417(13)$ & $9.35(8)$ & $8.40(5)$ & $1.482(7)$ \\
\hline QB4ASM2 & $1.328(4)$ & $4.644(8)$ & $6.168(20)$ & $9.63(14)$ & $8.67(6)$ & $1.563(15)$ \\
\hline QB4ASM3 & $1.389(4)$ & $4.418(9)$ & $6.139(21)$ & $10.12(7)$ & $8.83(10)$ & $1.611(12)$ \\
\hline QB4ASM4 & $1.504(8)$ & $4.027(8)$ & $6.06(3)$ & $10.10(15)$ & $8.86(14)$ & $1.647(23)$ \\
\hline QB4ASM5 & $1.729(12)$ & $3.516(9)$ & $6.08(5)$ & $10.35(16)$ & $9.93(12)$ & $1.743(28)$ \\
\hline QB4ASM6 & $1.879(19)$ & $3.170(8)$ & $5.96(6)$ & $11.4(4)$ & & $1.70(3)$ \\
\hline QB5ASM1 & $1.482(6)$ & $4.165(8)$ & $6.170(29)$ & $10.35(13)$ & $8.98(9)$ & $1.605(16)$ \\
\hline QB5ASM2 & $1.805(28)$ & $3.435(9)$ & $6.20(10)$ & $10.29(20)$ & & $1.73(5)$ \\
\hline
\end{tabular}


TABLE XX. Masses of $\mathrm{t}$ and at states, in units of $a$ and $f_{\mathrm{ps}}$, for each of the ensembles and bare masses $a m_{0}$. The Dirac fermions transform in the 2-index antisymmetric representation. In parentheses we report the statistical uncertainties.

\begin{tabular}{llllr}
\hline \hline Measurement & \multicolumn{1}{c}{$a m_{\mathrm{t}}$} & $m_{\mathrm{t}} / f_{\mathrm{ps}}$ & $a m_{\mathrm{at}}$ & $m_{\mathrm{at}} / f_{\mathrm{ps}}$ \\
\hline QB1ASM1 & $0.7454(17)$ & $5.970(24)$ & $1.210(15)$ & $9.69(12)$ \\
QB1ASM2 & $0.6850(18)$ & $5.876(23)$ & $1.132(19)$ & $9.71(17)$ \\
QB1ASM3 & $0.6452(22)$ & $5.828(29)$ & $1.127(25)$ & $10.18(23)$ \\
QB1ASM4 & $0.601(4)$ & $5.80(4)$ & $0.96(5)$ & $9.3(4)$ \\
QB1ASM5 & $0.568(6)$ & $5.69(6)$ & $1.05(4)$ & $10.6(4)$ \\
QB1ASM6 & $0.546(7)$ & $5.70(7)$ & $1.067(26)$ & $11.13(28)$ \\
QB2ASM1 & $0.6375(7)$ & $5.989(10)$ & $1.027(12)$ & $9.64(12)$ \\
QB2ASM2 & $0.5658(16)$ & $5.852(18)$ & $0.957(23)$ & $9.90(24)$ \\
QB2ASM3 & $0.5488(15)$ & $5.901(18)$ & $0.986(17)$ & $10.60(20)$ \\
QB2ASM4 & $5.876(24)$ & $0.988(11)$ & $11.11(12)$ \\
QB2ASM5 & $0.5228(19)$ & $5.91(3)$ & $0.94(3)$ & $11.0(4)$ \\
QB2ASM6 & & & $9.58(29)$ \\
QB3ASM1 & $5.99(3)$ & $0.781(24)$ & $10.65(10)$ \\
QB3ASM2 & $0.5022(29)$ & $6.016(25)$ & $0.829(8)$ & $10.85((29)$ \\
QB3ASM3 & & $0.04(4)$ & $0.799(21)$ & $11.2(5)$ \\
QB3ASM4 & $0.4884(25)$ & $6.10(6)$ & $0.77(3)$ & $9.49(12)$ \\
QB4ASM1 & $0.4682(19)$ & $0.415(13)$ & $0.782(9)$ & $9.93(15)$ \\
QB4ASM2 & $0.4447(24)$ & $6.14(4)$ & $0.712(10)$ & $10.38(14)$ \\
QB4ASM3 & $0.423(4)$ & $6.12(3)$ & $0.719(9)$ & $10.32(18)$ \\
QB4ASM4 & $0.5285(6)$ & $6.16(4)$ & $0.675(12)$ & $9.7(6)$ \\
QB4ASM5 & $0.4402(26)$ & $5.74(12)$ & $0.59(4)$ & $10.84(18)$ \\
QB4ASM6 & $0.4238(20)$ & $5.99(11)$ & & $11.30(24)$ \\
QB5ASM1 & $0.4028(22)$ & $6.15(5)$ & $0.585(9)$ & $0.548(11)$ \\
QB5ASM2 & $0.349(7)$ & $6.32(7)$ & & \\
\hline \hline
\end{tabular}

[1] D. B. Kaplan and H. Georgi, $S U(2) \times U(1)$ Breaking by vacuum misalignment, Phys. Lett. 136B, 183 (1984).

[2] H. Georgi and D. B. Kaplan, Composite Higgs and custodial SU(2), Phys. Lett. 145B, 216 (1984).

[3] M. J. Dugan, H. Georgi, and D. B. Kaplan, Anatomy of a composite Higgs model, Nucl. Phys. B254, 299 (1985).

[4] G. Aad et al. (ATLAS Collaboration), Observation of a new particle in the search for the Standard Model Higgs boson with the ATLAS detector at the LHC, Phys. Lett. B 716, 1 (2012).

[5] S. Chatrchyan et al. (CMS Collaboration), Observation of a new boson at a mass of $125 \mathrm{GeV}$ with the CMS experiment at the LHC, Phys. Lett. B 716, 30 (2012).

[6] K. Agashe, R. Contino, and A. Pomarol, The minimal composite Higgs model, Nucl. Phys. B719, 165 (2005).

[7] R. Contino, L. Da Rold, and A. Pomarol, Light custodians in natural composite Higgs models, Phys. Rev. D 75, 055014 (2007).
[8] R. Barbieri, B. Bellazzini, V. S. Rychkov, and A. Varagnolo, The Higgs boson from an extended symmetry, Phys. Rev. D 76, 115008 (2007).

[9] P. Lodone, Vector-like quarks in a composite Higgs model, J. High Energy Phys. 12 (2008) 029.

[10] D. Marzocca, M. Serone, and J. Shu, General composite Higgs models, J. High Energy Phys. 08 (2012) 013.

[11] C. Grojean, O. Matsedonskyi, and G. Panico, Light top partners and precision physics, J. High Energy Phys. 10 (2013) 160.

[12] G. Ferretti and D. Karateev, Fermionic UV completions of composite Higgs models, J. High Energy Phys. 03 (2014) 077.

[13] G. Cacciapaglia and F. Sannino, Fundamental composite (Goldstone) Higgs dynamics, J. High Energy Phys. 04 (2014) 111.

[14] A. Arbey, G. Cacciapaglia, H. Cai, A. Deandrea, S. Le Corre, and F. Sannino, Fundamental composite electroweak 
dynamics: Status at the LHC, Phys. Rev. D 95, 015028 (2017).

[15] L. Vecchi, A dangerous irrelevant UV-completion of the composite Higgs, J. High Energy Phys. 02 (2017) 094.

[16] G. Panico and A. Wulzer, The composite NambuGoldstone Higgs, Lect. Notes Phys. 913, 1 (2016).

[17] G. Ferretti, Gauge theories of partial compositeness: Scenarios for Run-II of the LHC, J. High Energy Phys. 06 (2016) 107.

[18] A. Agugliaro, O. Antipin, D. Becciolini, S. De Curtis, and M. Redi, UV complete composite Higgs models, Phys. Rev. D 95, 035019 (2017).

[19] T. Alanne, D. B. Franzosi, and M. T. Frandsen, A partially composite Goldstone Higgs, Phys. Rev. D 96, 095012 (2017).

[20] F. Feruglio, B. Gavela, K. Kanshin, P. A. N. Machado, S. Rigolin, and S. Saa, The minimal linear sigma model for the Goldstone Higgs, J. High Energy Phys. 06 (2016) 038.

[21] T. DeGrand, M. Golterman, E. T. Neil, and Y. Shamir, One-loop Chiral perturbation theory with two fermion representations, Phys. Rev. D 94, 025020 (2016).

[22] S. Fichet, G. von Gersdorff, E. Pontòn, and R. Rosenfeld, The excitation of the Global symmetry-breaking vacuum in composite Higgs models, J. High Energy Phys. 09 (2016) 158.

[23] J. Galloway, A. L. Kagan, and A. Martin, A UV complete partially composite-pNGB Higgs, Phys. Rev. D 95, 035038 (2017).

[24] C. Csaki, T. Ma, and J. Shu, Maximally Symmetric Composite Higgs Models, Phys. Rev. Lett. 119, 131803 (2017).

[25] M. Chala, G. Durieux, C. Grojean, L. de Lima, and O. Matsedonskyi, Minimally extended SILH, J. High Energy Phys. 06 (2017) 088.

[26] C. Csaki, T. Ma, and J. Shu, Trigonometric Parity for Composite Higgs Models, Phys. Rev. Lett. 121, 231801 (2018).

[27] V. Ayyar, T. DeGrand, M. Golterman, D. C. Hackett, W. I. Jay, E. T. Neil, Y. Shamir, and B. Svetitsky, Spectroscopy of SU(4) composite Higgs theory with two distinct fermion representations, Phys. Rev. D 97, 074505 (2018).

[28] T. Alanne, D. B. Franzosi, M. T. Frandsen, M. L. A. Kristensen, A. Meroni, and M. Rosenlyst, Partially composite Higgs models: Phenomenology and RG analysis, J. High Energy Phys. 01 (2018) 051.

[29] V. Ayyar, T. Degrand, D. C. Hackett, W. I. Jay, E. T. Neil, Y. Shamir, and B. Svetitsky, Baryon spectrum of SU(4) composite Higgs theory with two distinct fermion representations, Phys. Rev. D 97, 114505 (2018).

[30] V. Ayyar, T. DeGrand, D. C. Hackett, W. I. Jay, E. T. Neil, Y. Shamir, and B. Svetitsky, Finite-temperature phase structure of $\mathrm{SU}(4)$ gauge theory with multiple fermion representations, Phys. Rev. D 97, 114502 (2018).

[31] C. Cai, G. Cacciapaglia, and H. H. Zhang, Vacuum alignment in a composite 2HDM, J. High Energy Phys. 01 (2019) 130.

[32] A. Agugliaro, G. Cacciapaglia, A. Deandrea, and S. De Curtis, Vacuum misalignment and pattern of scalar masses in the $\mathrm{SU}(5) / \mathrm{SO}(5)$ composite Higgs model, J. High Energy Phys. 02 (2019) 089.
[33] V. Ayyar, T. DeGrand, D. C. Hackett, W. I. Jay, E. T. Neil, Y. Shamir, and B. Svetitsky, Partial compositeness and baryon matrix elements on the lattice, Phys. Rev. D 99, 094502 (2019).

[34] G. Cacciapaglia, S. Vatani, T. Ma, and Y. Wu, Towards a fundamental safe theory of composite Higgs and dark matter, arXiv:1812.04005.

[35] O. Witzel, Review on composite Higgs models, Proc. Sci., LATTICE2018 (2019) 006 [arXiv:1901.08216].

[36] G. Cacciapaglia, G. Ferretti, T. Flacke, and H. Serôdio, Light scalars in composite Higgs models, Front. Phys. 7, 22 (2019).

[37] V. Ayyar, M. F. Golterman, D. C. Hackett, W. Jay, E. T. Neil, Y. Shamir, and B. Svetitsky, Radiative contribution to the composite-Higgs potential in a tworepresentation Lattice model, Phys. Rev. D 99, 094504 (2019).

[38] G. Cossu, L. Del Debbio, M. Panero, and D. Preti, Strong dynamics with matter in multiple representations: SU(4) gauge theory with fundamental and sextet fermions, Eur. Phys. J. C 79, 638 (2019).

[39] G. Cacciapaglia, H. Cai, A. Deandrea, and A. Kushwaha, Composite Higgs and dark matter model in $\mathrm{SU}(6) / \mathrm{SO}(6)$, J. High Energy Phys. 10 (2019) 035.

[40] D. B. Franzosi and G. Ferretti, Anomalous dimensions of potential top-partners, SciPost Phys. 7, 027 (2019).

[41] E. Katz, A.E. Nelson, and D. G.E. Walker, The intermediate Higgs, J. High Energy Phys. 08 (2005) 074.

[42] B. Gripaios, A. Pomarol, F. Riva, and J. Serra, Beyond the minimal composite Higgs model, J. High Energy Phys. 04 (2009) 070.

[43] J. Barnard, T. Gherghetta, and T. S. Ray, UV descriptions of composite Higgs models without elementary scalars, J. High Energy Phys. 02 (2014) 002.

[44] R. Lewis, C. Pica, and F. Sannino, Light asymmetric dark matter on the Lattice: SU(2) technicolor with two fundamental flavors, Phys. Rev. D 85, 014504 (2012).

[45] A. Hietanen, R. Lewis, C. Pica, and F. Sannino, Fundamental composite Higgs dynamics on the Lattice: SU(2) with two flavors, J. High Energy Phys. 07 (2014) 116.

[46] R. Arthur, V. Drach, M. Hansen, A. Hietanen, C. Pica, and F. Sannino, SU(2) gauge theory with two fundamental flavors: A minimal template for model building, Phys. Rev. D 94, 094507 (2016).

[47] R. Arthur, V. Drach, A. Hietanen, C. Pica, and F. Sannino, $S U(2)$ gauge theory with two fundamental flavours: Scalar and Pseudoscalar spectrum, arXiv:1607.06654.

[48] C. Pica, V. Drach, M. Hansen, and F. Sannino, Composite Higgs dynamics on the Lattice, EPJ Web Conf. 137, 10005 (2017).

[49] W. Detmold, M. McCullough, and A. Pochinsky, Dark nuclei. II. Nuclear spectroscopy in two-color QCD, Phys. Rev. D 90, 114506 (2014).

[50] J. W. Lee, B. Lucini, and M. Piai, Symmetry restoration at high-temperature in two-color and two-flavor lattice gauge theories, J. High Energy Phys. 04 (2017) 036.

[51] G. Cacciapaglia, H. Cai, A. Deandrea, T. Flacke, S. J. Lee, and A. Parolini, Composite scalars at the LHC: The Higgs, the sextet and the octet, J. High Energy Phys. 11 (2015) 201. 
[52] N. Bizot, M. Frigerio, M. Knecht, and J. L. Kneur, Nonperturbative analysis of the spectrum of meson resonances in an ultraviolet-complete composite-Higgs model, Phys. Rev. D 95, 075006 (2017).

[53] D. K. Hong, Very light dilaton and naturally light Higgs boson, J. High Energy Phys. 02 (2018) 102.

[54] M. Golterman and Y. Shamir, Effective potential in ultraviolet completions for composite Higgs models, Phys. Rev. D 97, 095005 (2018).

[55] V. Drach, T. Janowski, and C. Pica, Update on SU(2) gauge theory with $N_{F}=2$ fundamental flavours, EPJ Web Conf. 175, 08020 (2018).

[56] F. Sannino, P. Stangl, D. M. Straub, and A. E. Thomsen, Flavor physics and flavor anomalies in minimal fundamental partial compositeness, Phys. Rev. D 97, 115046 (2018).

[57] T. Alanne, N. Bizot, G. Cacciapaglia, and F. Sannino, Classification of NLO operators for composite Higgs models, Phys. Rev. D 97, 075028 (2018).

[58] N. Bizot, G. Cacciapaglia, and T. Flacke, Common exotic decays of top partners, J. High Energy Phys. 06 (2018) 065.

[59] D. B. Franzosi, G. Cacciapaglia, and A. Deandrea, Sigmaassisted natural composite Higgs, arXiv:1809.09146.

[60] H. Gertov, A. E. Nelson, A. Perko, and D. G. E. Walker, Lattice-friendly gauge completion of a composite Higgs with top partners, J. High Energy Phys. 02 (2019) 181.

[61] G. Cacciapaglia, S. Vatani, and C. Zhang, Composite Higgs meets Planck scale: Partial compositeness from partial unification, arXiv:1911.05454.

[62] E. Bennett, D. K. Hong, J. W. Lee, C.-J. D. Lin, B. Lucini, M. Piai, and D. Vadacchino, $\operatorname{Sp}(4)$ gauge theory on the lattice: Towards SU(4)/Sp(4) composite Higgs (and beyond), J. High Energy Phys. 03 (2018) 185.

[63] E. Bennett, D. K. Hong, J. W. Lee, C.-J. D. Lin, B. Lucini, M. Piai, and D. Vadacchino, Higgs compositeness in Sp $(2 \mathrm{~N})$ gauge theories: Resymplecticisation, scale setting and topology, EPJ Web Conf. 175, 08012 (2018).

[64] E. Bennett, D. K. Hong, J. W. Lee, C.-J. D. Lin, B. Lucini, M. Piai, and D. Vadacchino, Higgs compositeness in Sp $(2 \mathrm{~N})$ gauge theories: Determining the low-energy constants with lattice calculations, EPJ Web Conf. 175, 08011 (2018).

[65] E. Bennett, D. K. Hong, J. W. Lee, C.-J. D. Lin, B. Lucini, M. Piai, and D. Vadacchino, Higgs compositeness in Sp $(2 \mathrm{~N})$ gauge theories: The pure gauge model, EPJ Web Conf. 175, 08013 (2018).

[66] J. W. Lee, E. Bennett, D. K. Hong, C.-J. D. Lin, B. Lucini, M. Piai, and D. Vadacchino, Progress in the lattice simulations of $\mathrm{Sp}(2 \mathrm{~N})$ gauge theories, Proc. Sci., LATTICE2018 (2018) 192 [arXiv:1811.00276].

[67] E. Bennett, D. K. Hong, J. W. Lee, C.-J. D. Lin, B. Lucini, M. Piai, and D. Vadacchino, $\mathrm{Sp}(4)$ gauge theories on the lattice: $N_{f}=2$ dynamical fundamental fermions, J. High Energy Phys. 12 (2019) 053.

[68] E. Bennett, D. K. Hong, J. W. Lee, C.-J. D. Lin, B. Lucini, M. Piai, and D. Vadacchino, Meson spectrum of Sp(4) lattice gauge theory with two fundamental Dirac fermions, arXiv:1911.00437.
[69] M. Bando, T. Kugo, S. Uehara, K. Yamawaki, and T. Yanagida, Is rho Meson a Dynamical Gauge Boson of Hidden Local Symmetry?, Phys. Rev. Lett. 54, 1215 (1985).

[70] R. Casalbuoni, S. De Curtis, D. Dominici, and R. Gatto, Effective weak interaction theory with possible new vector resonance from a strong Higgs sector, Phys. Lett. 155B, 95 (1985).

[71] M. Bando, T. Kugo, and K. Yamawaki, Nonlinear realization and hidden local symmetries, Phys. Rep. 164, 217 (1988).

[72] R. Casalbuoni, S. De Curtis, D. Dominici, F. Feruglio, and R. Gatto, Vector and axial vector bound states from a strongly interacting electroweak sector, Int. J. Mod. Phys. A 04, 1065 (1989).

[73] M. Harada and K. Yamawaki, Hidden local symmetry at loop: A new perspective of composite gauge boson and chiral phase transition, Phys. Rep. 381, 1 (2003).

[74] H. Georgi, Vector realization of Chiral symmetry, Nucl. Phys. B331, 311 (1990).

[75] T. Appelquist, P. S. R. da Silva, and F. Sannino, Enhanced global symmetries and the chiral phase transition, Phys. Rev. D 60, 116007 (1999).

[76] M. Piai, A. Pierce, and J. G. Wacker, Composite vector mesons from QCD to the little Higgs, arXiv:hep-ph/ 0405242.

[77] D. B. Franzosi, G. Cacciapaglia, H. Cai, A. Deandrea, and M. Frandsen, Vector and axial-vector resonances in composite models of the Higgs boson, J. High Energy Phys. 11 (2016) 076.

[78] K. Holland, M. Pepe, and U. J. Wiese, The deconfinement phase transition of $\mathrm{Sp}(2)$ and $\mathrm{Sp}(3)$ Yang-Mills theories in $(2+1)$-dimensions and $(3+1)$-dimensions, Nucl. Phys. B694, 35 (2004).

[79] N. Cabibbo and E. Marinari, A new method for updating SU(N) matrices in computer simulations of gauge theories, Phys. Lett. 119B, 387 (1982).

[80] L. Del Debbio, A. Patella, and C. Pica, Higher representations on the lattice: Numerical simulations. SU(2) with adjoint fermions, Phys. Rev. D 81, 094503 (2010).

[81] M. Lüscher, Properties and uses of the Wilson flow in lattice QCD, J. High Energy Phys. 08 (2010) 071; Erratum, J. High Energy Phys.03 (2014) 92.

[82] M. Lüscher, Trivializing maps, the Wilson flow and the HMC algorithm, Commun. Math. Phys. 293, 899 (2010).

[83] M. Lüscher and P. Weisz, Perturbative analysis of the gradient flow in non-abelian gauge theories, J. High Energy Phys. 02 (2011) 051.

[84] K. Fujikawa, The gradient flow in $\lambda \phi^{4}$ theory, J. High Energy Phys. 03 (2016) 021.

[85] S. Borsanyi et al., High-precision scale setting in lattice QCD, J. High Energy Phys. 09 (2012) 010.

[86] Z. Fodor, K. Holland, J. Kuti, S. Mondal, D. Nogradi, and C. H. Wong, The lattice gradient flow at tree-level and its improvement, J. High Energy Phys. 09 (2014) 018.

[87] A. Ramos and S. Sint, Symanzik improvement of the gradient flow in lattice gauge theories, Eur. Phys. J. C 76, 15 (2016).

[88] C.-J. D. Lin, K. Ogawa, and A. Ramos, The Yang-Mills gradient flow and SU(3) gauge theory with 12 massless 
fundamental fermions in a colour-twisted box, J. High Energy Phys. 12 (2015) 103.

[89] M. Lüscher and S. Schaefer, Lattice QCD without topology barriers, J. High Energy Phys. 07 (2011) 036.

[90] D. Galletly, M. Gurtler, R. Horsley, H. Perlt, P. E. L. Rakow, G. Schierholz, A. Schiller, and T. Streuer, Hadron spectrum, quark masses and decay constants from light overlap fermions on large lattices, Phys. Rev. D 75, 073015 (2007).

[91] R. Brower, S. Chandrasekharan, J. W. Negele, and U. J. Wiese, QCD at fixed topology, Phys. Lett. B 560, 64 (2003).

[92] C. Bernard, and D. Toussaint, Effects of nonequilibrated topological charge distributions on pseudoscalar meson masses and decay constants, Phys. Rev. D 97, 074502 (2018).

[93] P. A. Boyle, A. Juttner, C. Kelly, and R. D. Kenway, Use of stochastic sources for the lattice determination of light quark physics, J. High Energy Phys. 08 (2008) 086.

[94] G. Martinelli and Y. C. Zhang, The connection between local operators on the Lattice and in the continuum and its relation to Meson decay constants, Phys. Lett. 123B, 433 (1983).

[95] G. P. Lepage and P. B. Mackenzie, On the viability of lattice perturbation theory, Phys. Rev. D 48, 2250 (1993).

[96] B. Sheikholeslami and R. Wohlert, Improved continuum limit Lattice action for QCD with Wilson Fermions, Nucl. Phys. B259, 572 (1985).

[97] G. Rupak and N. Shoresh, Chiral perturbation theory for the Wilson lattice action, Phys. Rev. D 66, 054503 (2002).

[98] S. R. Sharpe and R. L. Singleton, Jr., Spontaneous flavor and parity breaking with Wilson fermions, Phys. Rev. D 58, 074501 (1998).

[99] M. Lüscher, S. Sint, R. Sommer, and P. Weisz, Chiral symmetry and $\mathrm{O}(\mathrm{a})$ improvement in lattice QCD, Nucl. Phys. B478, 365 (1996).

[100] K. Symanzik, Continuum limit and improved action in Lattice theories. 1. Principles and $\phi^{4}$ theory, Nucl. Phys. B226, 187 (1983).

[101] C. W. Bernard and M. F. L. Golterman, Finite volume two pion energies and scattering in the quenched approximation, Phys. Rev. D 53, 476 (1996).

[102] C.-J. D. Lin, G. Martinelli, E. Pallante, C. T. Sachrajda, and G. Villadoro, Finite volume two pion amplitudes in the $I=0$ channel, Phys. Lett. B 553, 229 (2003).

[103] C.-J. D. Lin, G. Martinelli, E. Pallante, C. T. Sachrajda, and G. Villadoro, Finite volume partially quenched two pion amplitudes in the $I=0$ channel, Phys. Lett. B 581, 207 (2004).

[104] D. Nogradi and L. Szikszai, The flavor dependence of $m_{\rho} / f_{\pi}$, J. High Energy Phys. 05 (2019) 197.

[105] D. Nogradi and L. Szikszai, The model dependence of $m_{Q} / f_{\pi}$, arXiv:1912.04114.

[106] K. Kawarabayashi and M. Suzuki, Partially Conserved Axial Vector Current and the Decays of Vector Mesons, Phys. Rev. Lett. 16, 255 (1966).

[107] Riazuddin and Fayyazuddin, Algebra of current components and decay widths of rho and $\mathrm{K}^{*}$ mesons, Phys. Rev. 147, 1071 (1966).
[108] F. Sannino, Conformal Windows of SP(2N) and $\mathrm{SO}(\mathrm{N})$ gauge theories, Phys. Rev. D 79, 096007 (2009).

[109] T. A. Ryttov and R. Shrock, Infrared fixed point physics in $\mathrm{SO}\left(N_{c}\right)$ and $\operatorname{Sp}\left(N_{c}\right)$ gauge theories, Phys. Rev. D 96, 105015 (2017).

[110] B. S. Kim, D. K. Hong, and J. W. Lee, Into the conformal window: Multirepresentation gauge theories, Phys. Rev. D 101, 056008 (2020).

[111] T. DeGrand and Y. Shamir, One-loop anomalous dimension of top-partner hyperbaryons in a family of composite Higgs models, Phys. Rev. D 92, 075039 (2015).

[112] A. Athenodorou, E. Bennett, G. Bergner, and B. Lucini, Infrared regime of SU(2) with one adjoint Dirac flavor, Phys. Rev. D 91, 114508 (2015).

[113] G. Ecker, J. Gasser, H. Leutwyler, A. Pich, and E. de Rafael, Chiral Lagrangians for massive spin 1 fields, Phys. Lett. B 223, 425 (1989).

[114] J. Bijnens and E. Pallante, On the tensor formulation of effective vector Lagrangians and duality transformations, Mod. Phys. Lett. A 11, 1069 (1996).

[115] P. C. Bruns and U. G. Meissner, Infrared regularization for spin-1 fields, Eur. Phys. J. C 40, 97 (2005).

[116] D. Elander, M. Piai, and J. Roughley, Holographic glueballs from the circle reduction of Romans supergravity, J. High Energy Phys. 02 (2019) 101.

[117] S. R. Coleman and E. J. Weinberg, Radiative corrections as the origin of spontaneous symmetry breaking, Phys. Rev. D 7, 1888 (1973).

[118] M. Tanabashi et al. (Particle Data Group), Review of particle physics, Phys. Rev. D 98, 030001 (2018).

[119] L. Y. Glozman, Restoration of chiral and U(1)A symmetries in excited hadrons, Phys. Rep. 444, 1 (2007).

[120] M. Denissenya, L. Y. Glozman, and C. B. Lang, Isoscalar mesons upon unbreaking of chiral symmetry, Phys. Rev. D 91, 034505 (2015).

[121] L. Y. Glozman and M. Pak, Exploring a new SU(4) symmetry of meson interpolators, Phys. Rev. D 92, 016001 (2015).

[122] C. Rohrhofer, Y. Aoki, G. Cossu, H. Fukaya, L. Y. Glozman, S. Hashimoto, C. B. Lang, and S. Prelovsek, Approximate degeneracy of $J=1$ spatial correlators in high temperature QCD, Phys. Rev. D 96, 094501 (2017); Erratum, Phys. Rev. D 99, 039901 (2019).

[123] L. Glozman, Chiralspin symmetry and its implications for QCD, Universe 5, 38 (2019).

[124] C. Rohrhofer, Y. Aoki, G. Cossu, H. Fukaya, C. Gattringer, L. Ya. Glozman, S. Hashimoto, C. B. Lang, and S. Prelovsek, Symmetries of spatial meson correlators in high temperature QCD, Phys. Rev. D 100, 014502 (2019).

[125] L. Y. Glozman, Three regimes of QCD, arXiv:1907.01820.

[126] G. P. Engel, C. B. Lang, M. Limmer, D. Mohler, and A. Schäfer, QCD with two light dynamical chirally improved quarks: Mesons, Phys. Rev. D 85, 034508 (2012).

[127] J. Gratrex and R. Zwicky, Parity doubling as a tool for right-handed current searches, J. High Energy Phys. 08 (2018) 178.

[128] A. Abele et al. (Crystal Barrel Collaboration), Anti-proton proton annihilation at rest into $\mathrm{K}(\mathrm{L}) \mathrm{K}(\mathrm{S})$ pi0 pi0, Phys. Lett. B 415, 280 (1997). 
[129] R. D. Pisarski and F. Wilczek, Remarks on the Chiral phase transition in chromodynamics, Phys. Rev. D 29, 338 (1984).

[130] T. W. Chiu et al. (TWQCD Collaboration), Chiral symmetry and axial U(1) symmetry in finite temperature QCD with domain-wall fermion, Proc. Sci., LATTICE2013 (2014) 165 [arXiv:1311.6220].
[131] B. B. Brandt, M. Cè, A. Francis, T. Harris, H. B. Meyer, H. Wittig, and O. Philipsen, Testing the strength of the $\mathrm{U}_{A}(1)$ anomaly at the chiral phase transition in two-flavour QCD, arXiv:1904.02384].

[132] K. Suzuki et al. (JLQCD Collaboration), Axial U(1) symmetry, topology, and Dirac spectra at high temperature in $N_{f}=2$ lattice QCD, arXiv:1908.11684. 